\title{
Sound Encoding in the Mouse Cochlea: Molecular Physiology and Optogenetic Stimulation
}

\author{
PhD Thesis \\ in partial fulfilment of the requirements \\ for the degree "Doctor of Philosophy $(\mathrm{PhD}) /$ Dr. rer. nat." \\ in the Neuroscience Program \\ at the Georg August University Göttingen, \\ Faculty of Biology
}

submitted by

Zhizi Jing

born in

Chongqing, P.R.China

Göttingen, 2013 
Thesis committee:

Dr. Nicola Strenzke (Supervisor)

Junior Research Group "Auditory Systems Physiology"

Department of Otolaryngology

University Medical Center Göttingen

Prof. Dr. Tobias Moser

Department of Otolaryngology

University Medical Center Göttingen

Prof. Dr. Fred Wolf

Department of Nonlinear Dynamics

Max Planck Institute for Dynamics and Self-Organization, Göttingen

Prof. Dr. Martin Göpfert

Department of Cellular Neurobiology

Schwann-Schleiden Research Centre, Göttingen

Date of oral examination: Oct $23^{\text {rd }}, 2013$ 


\section{Declaration}

Hereby I declare that this thesis has been written independently and with no other sources and aids than quoted.

Zhizi Jing

Göttingen, August 30 ${ }^{\text {st }}$, 2013 


\section{Table of Content}

List of Figures vii

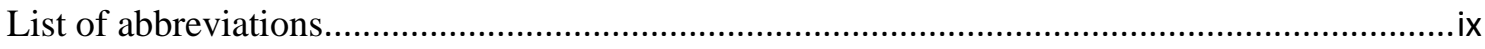

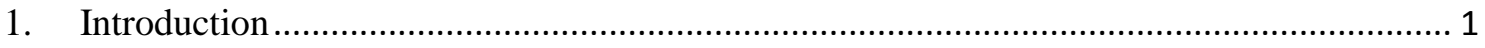

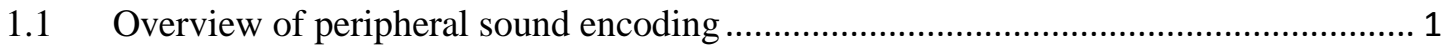

1.2 Studying sound encoding through auditory nerve fiber responses ............................... 3

1.3 Early postnatal development changes of ANF response properties.............................. 7

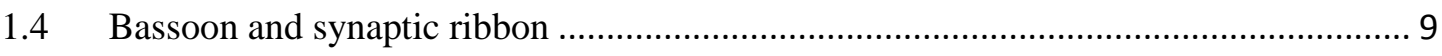

1.4.1 Synaptic ribbon and transmitter release at ribbon synapses................................ 9

1.4.2 Presynaptic scaffold protein Bassoon and mouse mutants................................... 11

1.4.3 Purpose of the study using Bassoon mutants .................................................... 12

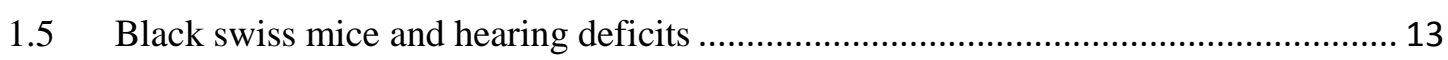

1.5.1 BLSW mice and hearing impairment phenotypes.......................................... 13

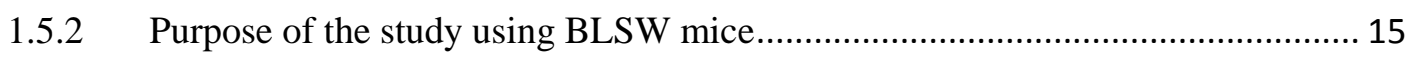

1.6 Cochlear implants in restoring the sense of hearing .............................................. 16

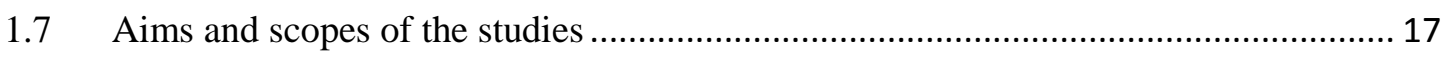

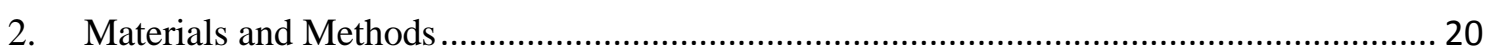

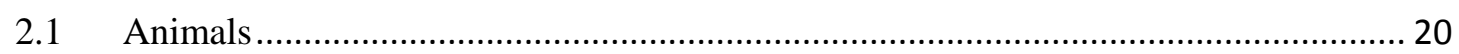

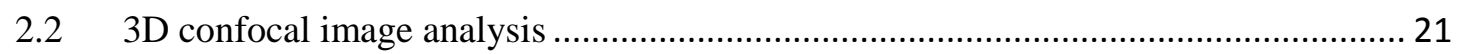

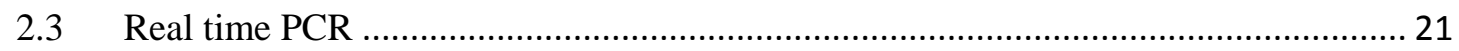

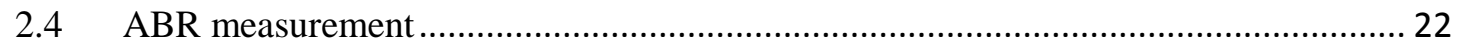

2.5 Single unit recording from mouse auditory nerve fibers ......................................... 22

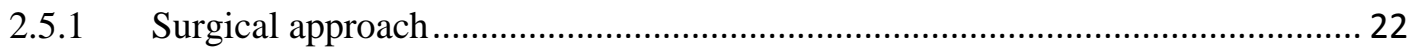

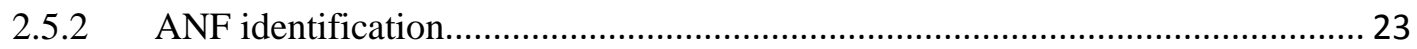

2.5.3 Acoustic system and signal processing …….................................................... 24

2.5.4 Stimulus and response measurements ............................................................... 24

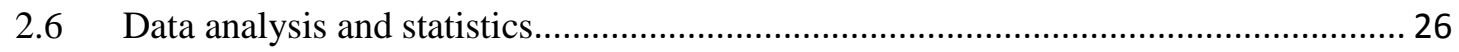

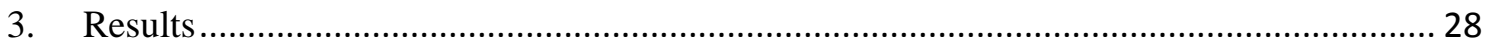

3.1 Auditory nerve fiber responses during early postnatal development in mice .............. 28

3.1.1 Developmental changes of spontaneous spiking activity of mouse ANFs before

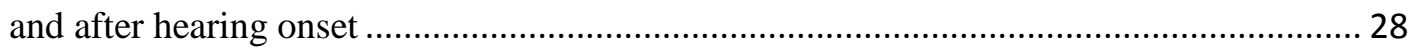

3.1.2 Maturation of frequency selectivity after hearing onset................................... 30

3.1.3 Maturation of response to pure tone bursts after hearing onset............................ 31

3.1.4 Development of rate-level function after hearing onset .................................... 32

3.2 Synaptic ribbon and presynaptic structure in sound encoding: lessons from Bassoon mutants 


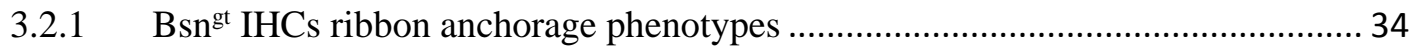

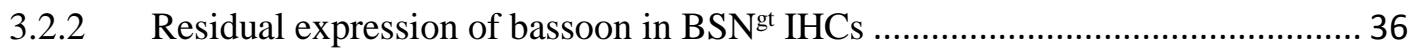

3.2.3 Ribbon-dependent effects on synaptic morphology and physiology in $\mathrm{Bsn}^{\mathrm{gt}} \mathrm{IHCs}$

3.2.4 Sound encoding phenotypes in $\mathrm{Bsn}^{\mathrm{gt}}$ and $\mathrm{Bsn}^{\Delta \mathrm{Ex} 4 / 5}$ ANFs ................................ 40

3.2.5 Auditory population responses in $\mathrm{Bsn}^{\mathrm{gt}}$ and $\mathrm{Bsn}^{\mathrm{EEx} 4 / 5}$ mice.................................. 48

3.3 Early onset progressive sensorineural hearing loss in Black Swiss Mice: auditory nerve responses in relation to underlying cochlear pathophysiology..................................... 50

3.3.1 Early onset hearing loss reflected in auditory population responses..................... 50

3.3.2 ANF response properties and cochlear lesion in BLSW mice ............................ 51

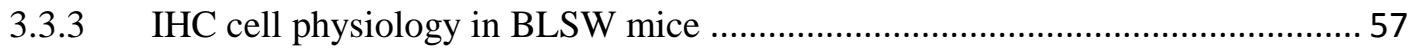

3.4 Optical stimulation of ChR2 expressing ANFs: endeavor towards rescuing hearing by light...

3.4.1 Optogenetic stimulation of ChR2 mice cochlea................................................ 59

3.4.2 AAV6 mediated expression of Catch variant in SGNs and light-evoked responses

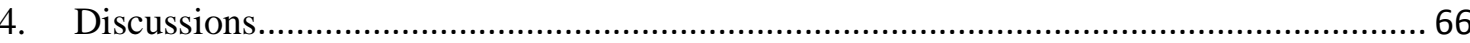

4.1 Auditory nerve fiber response properties during early postnatal development ............66

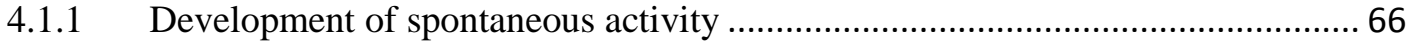

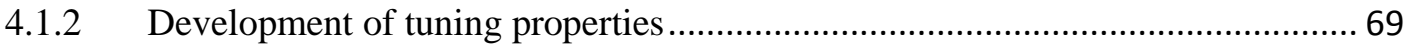

4.1.3 Development of responses to tone bursts ........................................................... 70

4.2 Bassoon and synaptic ribbon in sound encoding ...................................................... 72

4.2.1 Ribbon-occupied vs. ribbonless synapses ........................................................ 72

4.2.2 Discrepancy between cell physiology and single unit results ............................. 74

4.2.3 Direct consequences of Bassoon disruption on active zone function or effect of

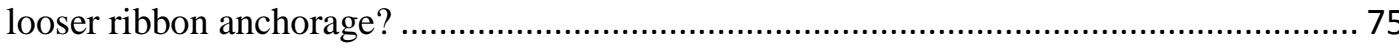

4.2.4 Alternative possible functions of Bassoon in ribbon synapses and conventional

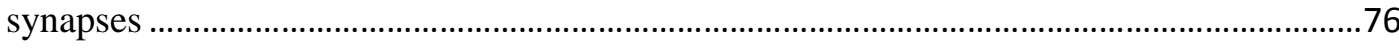

4.2.5 ANF sound coding deficit in Bsn mutants ...................................................... 77

4.3 Auditory nerve fiber responses in BLSW mice ….................................................... 79

4.3.1 Sound encoding phenotypes in BLSW cochlea …………................................... 79

4.3.2 Mechanisms underlying GIPC3 mutation on cochlear functions ......................... 81

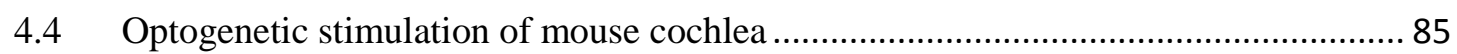

4.4.1 Optogenetic stimulation of auditory neurons ................................................... 85

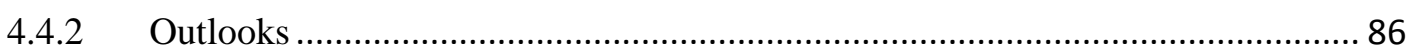

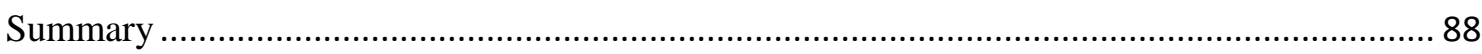

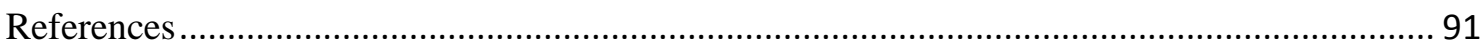




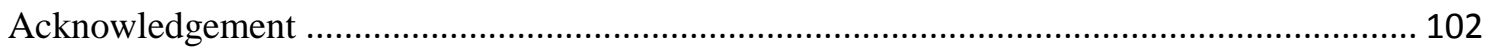

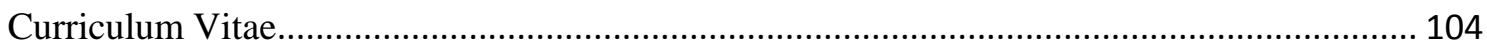

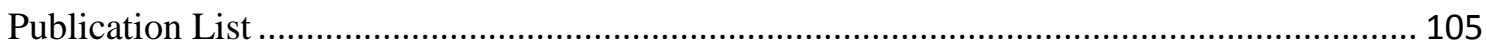




\section{List of Figures}

Figure 1.1: Illustration of basic response properties of ANFs: tuning curves and evoke responses.

Figure 1.2: Illustration of rate-level function and dynamic range of ANF.

Figure 1.3: Illustration of the Bassoon gene and two available mutations.

Figure 3.1: Putative ANF firing properties before hearing onset.

Figure 3.2: Cumulative distribution of spontaneous discharge rate of fibers before and after the onset of hearing.

Figure 3.3: The hearing thresholds decreased and the sharpness of tuning increased during early postnatal development after onset of hearing.

Figure 3.4: Development of ANF responses to suprathreshold tone bursts after the onset of hearing.

Figure 3.5: Changes of rate-level functions during early postnatal development.

Figure 3.6: $3 \mathrm{D}$ confocal imaging analysis of synaptic organization in $\mathrm{Bsn}^{\mathrm{gt}}$ mutants.

Figure 3.7: Leaky expression of bassoon in a subset of Bsn ${ }^{\mathrm{gt}}$ IHCs.

Figure 3.8: Ribbon dependent morphological effects in Bsn ${ }^{\mathrm{gt}} \mathrm{IHCs}$.

Figure 3.9: Effect of Bassoon disruption on synaptic functions.

Figure 3.10: Time pattern of spontaneous activities and lack of high spontaneous rate fibers in Bsn mutants

Figure 3.11: Normal auditory nerve fiber thresholds and sharpness of tuning of tuning curves.

Figure 3.12: The sound encoding of tone bursts were affected to a similar extent for both Bsn mutants. 
Figure 3.13: The time course of recovery from forward masking was similar in ANFs from all genotypes, indicating a normal time course of replenishment of the smaller synaptic vesicle pool.

Figure 3.14: Rate-level function and dynamic range.

Figure 3.15: Average synchronous index as a function of modulation frequency.

Figure 3.16: Auditory brainstem responses.

Figure 3.17: Auditory population responses in BLSW mice.

Figure 3.18: Tuning curve threshold and frequency selectivity in BLSW ANFs.

Figure 3.19: Spontaneous activity in BLSW ANFs.

Figure 3.20: Rate-level functions and dynamic range for BLSW and C57BL/6 ANFs.

Figure 3.21: Evoked responses of BLSW and C57BL/6 ANFs to $50 \mathrm{~ms}$ superathreshold tone bursts at $\mathrm{CF}$.

Figure 3.22: Recovery from adaptation assessed using forward-masking paradigm.

Figure 3.23: Average maximum synchronous index (SI) as a function of modulation frequency of 15 BLSW and 7 C57BL/6 ANFs.

Figure 3.24: Microelectrode recordings from individual neurons of the auditory pathway in response to optogenetic stimulation.

Figure 3.25: Analysis of responses from single neurons in Catch-transfected mice by optogenetic stimulation.

Figure 3.26: Acoustic and optogenetic stimulation confirmed the activation of neurons in the auditory pathway.

Figure 3.27: Response of neurons to different stimulus rates. 


\section{List of abbreviations}

\begin{tabular}{|l|l|}
\hline AAV6 & Adenoassociated-virus 6 \\
\hline ABI & Auditory brainstem implant \\
\hline ABR & Auditory brainstem response \\
\hline AM & Amplitude modulation \\
\hline ANF & Auditory nerve fiber \\
\hline AP & Action potential \\
\hline AVCN & Anterior ventral cochlear nucleus \\
\hline AZ & Active zone \\
\hline BLSW & Black Swiss Mice \\
\hline BM & Basilar membrane \\
\hline Bsn & Bassoon \\
\hline CAP & Compound action potential \\
\hline CF & Characteristic frequency \\
\hline ChR2 & Channelrhodopsin 2 \\
\hline CI & Cochlear implant \\
\hline CtBP2 & C-terminal binding protein 2 \\
\hline CV & Coefficient of variation \\
\hline DPOAE & Distortion product otoacoustic emissions \\
\hline EM & Electron microscopy \\
\hline EP & Endocochlear potential \\
\hline FSL & First spike latency \\
\hline IC & Inferior colliculus \\
\hline IHC & Inner hair cell \\
\hline ISI & Inter spike interval \\
\hline LOC & Lateral olivocochlear \\
\hline oABR & Optical auditory brainstem response \\
\hline OHC & Outer hair cell \\
\hline PSTH & Post stimulus time histogram \\
\hline QTL & Quantitative trait locus \\
\hline RRP & Readily releasable pool \\
\hline SGN & Spiral ganglion neuron \\
\hline SNHL & Sensorineural hearing loss \\
\hline SPL & Sound pressure level \\
\hline SR & Spontaneous firing rate \\
\hline & \\
\hline & \\
\hline
\end{tabular}




\section{Introduction}

\subsection{Overview of peripheral sound encoding}

The auditory sense is of great importance for mammalians to survive and communicate (Bregman 1990). Maximizing the power of bilateral hearing for tasks like sound source localization and categorization requires rapid and precise processing of spectrotemporal information of incoming acoustic stimuli. The peripheral hearing organ in mammalians is capable of preserving interaural time differences with microsecond precision (Geisler 1998). How is the peripheral ear capable of performing such marvel? Nature has evolved a highly specialized and efficient peripheral mammalian hearing organ to faithful transduce acoustic air pressure waveforms into continuous spike trains in tens of thousands parallel auditory nerve fibers (ANFs). Investigating the mechanisms of the peripheral auditory system may ultimately demystify the central processing mechanisms. This thesis is about studying the first stage of sound encoding in the mouse ANFs to indirectly infer related cochlear physiology. The peripheral auditory system is not just a sensor but rather a device composed of several components. Before touching the main topics of the thesis, the anatomy and physiology of the peripheral sound encoding apparatus is firstly reviewed.

Acoustic signals are first mechanically collected by the external and middle ear. The external ear channels the energy of the incoming acoustic wave onto the tympanic membrane. The middle ear connects the tympanic membrane with the oval window via three small ossicles, namely malleus, incus and stapes. The vibration of the tympanic membrane and ossicle chain is then transmitted into the fluid (the perilymph) in the cochlea through oval window. The tiny mechanical devices in the middle ear function as a lever system to match the low air impedance to high impedance of the inner ear fluid so that the energy not being reflected away (Geisler 1998, Schnupp, Nelken et al. 2011). At these stages, the external and middle ear together work as linear mechanical transducers which transform acoustic vibration of air molecules into travelling waves of the inner ear fluid, unless the middle ear muscles are activated by feedback mechanisms to attenuate the high intensity sound reaching the cochlea (Huttenbrink 1989). 
In the inner ear, the mechanical propagation of travelling waves in the cochlear duct is detected by a sensory epithelium, the organ of Corti, resting on the basilar membrane (BM). The travelling wave is decomposed along the cochlear axis into different frequency components due to the tonotopic properties of the basilar membrane and inertial mass of the fluid (Robles and Ruggero 2001, Olson, Duifhuis et al. 2012). The basal part is most sensitive to stimulation by high frequency sounds while the apex region analyzes low frequency sounds. Thus, the basilar membrane functions as a Fourier analyzer for frequency decomposition of sound signal. The organ of Corti harbors two type of hair cells, outer hair cells (OHCs) and inner hair cells (IHCs). IHCs form one row and OHCs three rows along the cochlear tonotopic axis. The OHCs non-linearly amplify the vibration of basilar membrane (Liberman, Gao et al. 2002, Ashmore 2008, Dallos, Wu et al. 2008), while the IHCs are the genuine sensory cells transmitting the mechanical vibration to spiral ganglion neurons (SGNs) via ribbon synapses (Ciuman 2011). Vibration-driven deflection of IHC stereocilia leads to the opening or closing of mechanotransduction channels on hair bundles, and the resulting cation influx generates the receptor potential of the IHCs membrane (Patuzzi and Sellick 1983). At active zones of IHCs, voltage gated $\mathrm{Ca}^{2+}$ channels controls $\mathrm{Ca}^{2+}$ signals for triggering neurotransmitter release (Brandt, Khimich et al. 2005, Frank, Rutherford et al. 2010). Glutamate release into the synaptic junction between IHCs and the peripheral axon of the SGNs binds to AMPA type receptors, generating neural spikes that propagate in the SGNs (Glowatzki and Fuchs 2002, Fuchs, Glowatzki et al. 2003). In complement to the frequency analyzer function of the basilar membrane, the synaptic junction between IHC and SGN is specialized for high fidelity temporal processing (Moser, Neef et al. 2006, Parsons 2006). The cell bodies of SGNs lie in a bony structure, the modiolus of the cochlea. The central axons of spiral ganglion neurons comprise ANFs, which then project to the cochlear nucleus through internal auditory meatus. Those fibers then branch to innervate many different types of cells throughout the cochlear nucleus. Each IHC contacts 10 30 afferent fibers (Liberman, Dodds et al. 1990), making parallel channels sending information to the central nervous system. 


\subsection{Studying sound encoding through auditory nerve fiber responses}

Studying the sound encoding in the cochlea is a challenging because assessing the working apparatus without disrupting normal functional states of the hearing organ is difficult. The sound encoding process in the cochlea can be divided into four stages: (1) a linear mechanical filter of basilar membrane; (2) a nonlinear transducer action of mechanical amplification of OHCs; (3) synaptic transmission of IHC synapses; (4) afferent fiber spike generation. The first stage mechanical filter can be approximated as a time-invariant linear system, thus described by gamma-tone impulse responses. Several other different experimental approaches exist to understand the cochlear physiology of the second and third stages. Non-invasive methods including measurements of distortion product otoacoustic emissions (DPOAE) and auditory brainstem responses (ABRs) and compound action potential (CAP) recordings near the round window are widely used to assess the overall status of the cochlea. Because gross potentials obtained from those methods come from multiple sources, they cannot provide accurate inference about physiology status at the individual hair cell and ANF level. Recently experimental approaches like using isolated explanted organs of Corti have advanced studies on nonlinear properties of OHCs and IHCs, as well as spike generation mechanism of spiral ganglion neurons (Rutherford, Chapochnikov et al. 2012). However, results from in vitro studies do not necessarily represent the physiological status in vivo. Computational modeling is also used in the study of cochlear sound encoding, but it requires assumptions concerning the physiological process which introduce uncertainty. Experimental data using different approaches need to be combined to constrain modeling by physiologically plausible assumptions about sound encoding. ANF responses, as the first stage in the neural processing underlying hearing, can provide valuable information about cochlear mechanisms of sound encoding and provide data to further constrain cochlear modeling studies. Therefore, another widely used approach to gain insight into the process is to record single unit activity from axons of ANFs.

The studies of single ANF responses started in the 1950s. Initially, the cat was chosen as the experimental species for those studies, mainly because of the anatomical, physiological and behavioral data available at that time (Kiang 1965). The general structure and function of the cochlea and the auditory nerve are similar among mammals. This is demonstrated in subsequent studies using other mammals including guinea pig 
(Evans 1972), rabbit (Borg, Engström et al. 1988), rat (el Barbary 1991, el Barbary 1991) and mouse (Taberner and Liberman 2005), which have revealed remarkable similarities in many aspect of basic ANF response properties. However, it is important to take into account differences in the acoustic characteristics of the peripheral apparatus of different species, which strongly influence the hearing range and thresholds but have less or no impact on IHC synaptic function. Major findings on ANF response properties relevant to the thesis are summarized below:

(1) Spontaneous activity of ANFs: There are approximately 50,000 cat fibers (Gacek and Rasmussen, 1961). The major findings from cat studies were that ANFs fire action potentials spontaneously in a stochastic manner. The time pattern can be described as a Poisson process with a refractoriness function. ANFs from one IHC can have different spontaneous firing rates (SR). It seems that in cat, the spontaneous firing rate is correlated with the morphology and location of the afferent innervation around IHCs. High SR fibers tend to innervate the pillar side of IHCs and they have more mitochondria and larger axon diameters while low SR fibers mainly connect the modiolar side of IHCs, with smaller amount of mitochondrial and smaller axon diameters (Liberman 1982, Liberman and Oliver 1984, Liberman, Wang et al. 2011). Fibers with different SR have also been shown to receive input from AZs occupied by synaptic ribbons of different size and complexity (Merchan-Perez and Liberman 1996). Functionally, low spontaneous rate fibers have lower sensitivity and wider dynamic range while high spontaneous rate fibers have high sensitivity and narrower dynamic range (Liberman 1978, Taberner and Liberman 2005). The distributions of cat, guinea pig, chinchilla and rabbit ANFs are bimodal, but this is not the case in rat, mouse and basal turn of gerbils (Taberner and Liberman 2005). Therefore, mouse ANFs cannot be classified into two/three groups like that in cat. However, mouse ANFs still show heterogeneity in level of spontaneous activity, ranging from completely silent up to $120 \mathrm{sp} / \mathrm{s}$ (Taberner and Liberman 2005). The origin of the SR heterogeneity is not clear. It can be mediated by presynaptic, postsynaptic mechanisms or efferent modulation. Unfortunately, direct methods to assess the mechanisms of spontaneous rate heterogeneity are currently not available.

(2) Tuning curves and frequency selectivity: The frequency selectivity of ANFs is based on the frequency decomposition of acoustic signals along the tonotopic axis. The 
passive BM responses provide a broad tuning. Subsequent nonlinear amplification of OHCs provide a sharp tuning tip around characteristic frequency for each ANF (Figure 1.1A). Perfusion of ototoxic drugs into the cochlea or noise trauma can result in a change in the shape of ANF tuning curves (Kiang, Liberman et al. 1986). Thus it is possible to differentiate the OHC amplification problem by looking at the ANF responses.

A

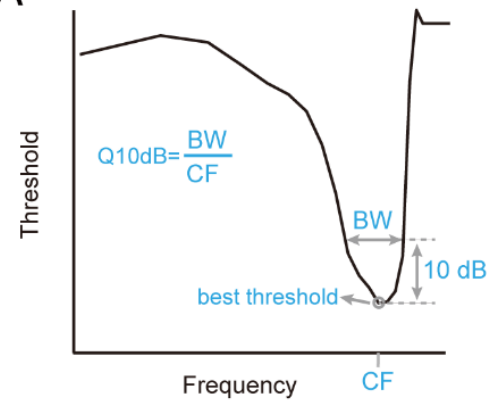

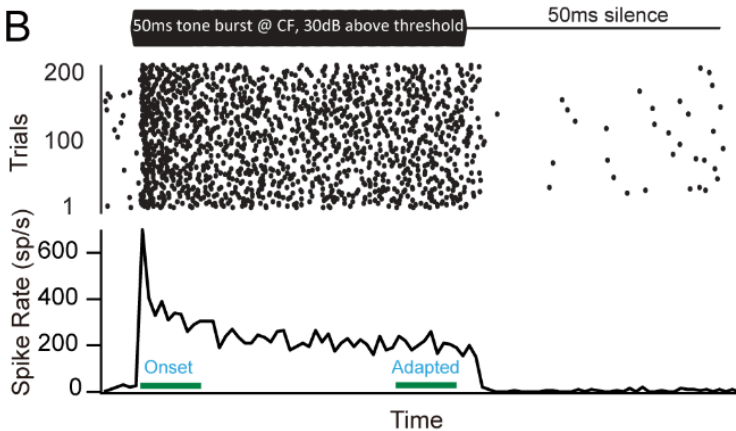

Figure 1.1: Illustration of basic response properties of ANFs: tuning curves and evoked responses. (A) Tuning curve shows the frequency selectivity of each ANF. The most sensitive frequency is defined as characteristic threshold. The sharpness of tuning is quantified as the bandwidth (BW) of tuning curve $10 \mathrm{~dB}$ above best threshold divided by the characteristic frequency. A larger Q10 dB value indicates sharper tuning/better frequency selectivity. (B) Upper panel: raster plot of a representative ANF response to 200 repetitions of tone bursts presented at characteristic frequency, $30 \mathrm{~dB}$ above threshold. Lower panel: post-stimulus time histogram (PSTH) of the spike times in response to tone bursts. The average spike rates in a $10 \mathrm{~ms}$ window during the initial transient response and later steady state were quantified as onset and adapted rate, respectively.

(3) Responses to tone bursts: In response to brief pure tones, ANFs fire a train of action potentials. Adaptation of the firing rate is one prominent feature of the response (Kiang 1965). A typical response of ANF to tone bursts goes through four stages. First, at the stimulus onset, there is a transient onset response, during which period the firing probability reaches maximum for the first few milliseconds of the tone. After the transient response, there is a decay stage, during which the discharge rate gradually adapts to a steady-state level. When the stimulus is over, the discharge rate drops sharply to levels below the spontaneous rate. During the last stage, the discharge then gradually recovers to its spontaneous activity level (Figure 1.1B). Analysis of the time course of adaptation have revealed the existence of multiple exponentiallydecaying components in different timescales (Westerman and Smith 1984, Yates, 
Robertson et al. 1985). The time course of adaptation can be described by fitting twoexponentially decaying components and a constant term to the PSTH. Depending on the stimulus duration and bin size used to construct post-stimulus time histogram (PSTH) for quantifying adaptation, different components of adaptation can be emphasized. For example, histogram window smaller than $1 \mathrm{~ms}$ or less emphasize on rapid adaptation during stimulus onset, as well as another slower short-term adapting component with time constant about 10-20 ms when stimulated with $50 \mathrm{~ms}$ tone bursts in mouse ANFs (Buran, Strenzke et al. 2010). Because the IHCs receptor potential does not adapt in response to tonal stimuli (Russell and Sellick 1978), the most likely location of adaptation is between of receptor potential and spike generation in ANF. Different biological processes can produce those different adapting components. For instance, a series of replenishment and diffusion steps involved in the vesicle release (Furukawa and Matsuura 1978, Moser and Beutner 2000, Sumner, Lopez-Poveda et al. 2002, Sumner, Lopez-Poveda et al. 2003, Nouvian, Beutner et al. 2006, Goutman and Glowatzki 2007), binding kinetics of glutamate to receptors and refractoriness of ANF spiking can contribute to different exponential components. It has been suggested that one important onset adapting component at the beginning of the stimulus is refractoriness of neurotransmitter release process and/or postsynaptic spiking (Berry and Meister 1998). Evidence suggests that short-term adaptation is mainly of presynaptic origin, reflecting the depletion of the presynaptic pool of vesicles (Moser and Beutner 2000, Singer and Diamond 2006). Subsequent recovery from adaptation reflects the replenishment of vesicles (Spassova, Avissar et al. 2004). Therefore, it is possible to infer the working status of the synaptic vesicle pool dynamics from ANF responses.

(4) Rate-level function and hair cell lesions: The firing rate of one ANF can be saturated with increasing sound intensity within a narrow range, which is usually about $20 \mathrm{~dB}$ in mouse ANFs (Taberner and Liberman 2005). Compared with single fiber dynamic range, our auditory system as a whole is capable of signaling stimulus in a much larger dynamic range (Plack 2005, Wen, Wang et al. 2009). One property related to this wide dynamic range is a shallow loudness increase, meaning the growth of perception of loudness given increasing sound stimulus (Viemeister 1983, Stillman, Zwislocki et al. 1993). Animals and humans suffering from sensorineuronal hearing loss (SNHL) or 
noise trauma have been shown to have steeper loudness recruitment (Heinz and Young 2004, Joris 2009). Steeper loudness recruitment typically reflects the loss of active compressive nonlinearity in the cochlea in the case of OHCs damage. ANFs have elevated thresholds and much steeper rate-level functions in this case (Yates 1990, Yates, Winter et al. 1990). Damage of IHCs results in a decrease in transduction currents or neurotransmitter release, thus a shallower rate-level function (Heinz and Young 2004, Joris 2009). ANFs recordings from cats and guinea pigs have confirmed this observation (Harrison 1981). However, the ANF firing rates alone failed to explain the shallower loudness recruitment (Heinz and Young 2004). In addition, it has been suggested that loudness recruitment is a central process, starting possibly at the level of cochlear nucleus neurons (Cai, Ma et al. 2009, Joris 2009). Nevertheless, IHCs and OHCs lesions can be reflected in the rate-level function of ANFs.

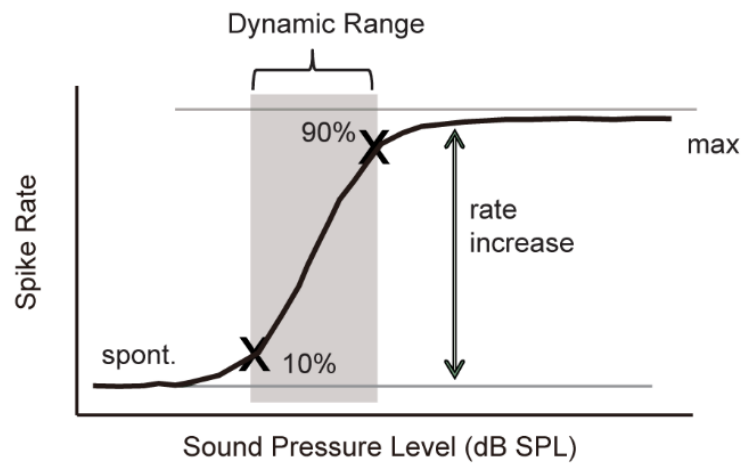

Figure 1.2: Illustration of rate-level function and dynamic range of ANF. The dynamic range is quantified as the range of sound pressure levels in which the rate-level function showed a rate increase between $10 \%$ and $90 \%$ of the difference between spontaneous and maximum rate.

\subsection{Early postnatal development changes of ANF response properties}

The mammalian peripheral auditory system undergoes rapid developmental changes around the age of hearing onset. Importantly, cochlea and ANFs in kittens are initially nearly unresponsive to acoustic stimuli, but acquire mature response properties during the first three postnatal weeks (Walsh and McGee 1987, Jones, Leake et al. 2007). In mice, studies suggest that the cochlea reaches a mature configuration by the $8^{\text {th }}$ to $10^{\text {th }}$ day 
(Mikaelian and Ruben 1965), and the auditory functions start between $9^{\text {th }}$ and $14^{\text {th }}$ day (Alford and Ruben 1963). However, no single unit studies have been performed to characterize developmental changes around onset of hearing in mice. The availability of immunohistochemistry, cell physiology and gross cochlear potential (Mikaelian, Alford et al. 1965, Mikaelian and Ruben 1965, Sadanaga and Morimitsu 1995) data from mice of similar ages makes it possible to correlate discharge properties with other development events in cochlea.

In this study, the following discharge properties of single fiber responses will be characterized in normal developing C57BL/6 mice during the period of hearing onset (p10 to $\mathrm{p} 21)$ :

(1) Spontaneous activity: ANFs fire action potentials spontaneously (Kiang 1965). The question of how the heterogeneity in spontaneous rates emerges is still not clear. In cat, ANFs with different SRs show different synaptic morphological features (Liberman 1980, Liberman 1982, Liberman, Wang et al. 2011), suggesting the existence of presynaptic mechanisms. It has been found that the spontaneous activity of ANFs is driven by spontaneous neurotransmitter release (Siegel and Relkin 1987, Siegel 1992, Glowatzki and Fuchs 2002). The observation of intrinsic heterogeneity in presynaptic calcium signals suggested a presynaptic origin of ANF SR heterogeneity (Frank, Khimich et al. 2009). Recent data show an emergence of strong synaptic $\mathrm{Ca}^{2+}$ influx at a subset of AZs over postnatal maturation, and a developmental increase in intensity and variance of Cav1.3 immunofluorescence (Wong, Jing et al. 2013). One purpose of this study is to investigate how ANF spontaneous activity changes during the period of presynaptic $\mathrm{Ca}^{2+}$ signaling maturation.

(2) Tuning and frequency selectivity: In kitten, the tuning and threshold of single ANFs reach mature levels at around second and third postnatal week (Romand 1983, Romand 1984). Currently, no single fiber data from mice are available on the development of tuning and frequency selectivity. Indirect methods, for example, using a masking approach and auditory brainstem responses have been used to approximate single fiber tuning properties (Saunders, Dolgin et al. 1980, Song, McGee et al. 2008). Results suggest that the passive tuning of basilar membrane 
reaches mature level by $\mathrm{p} 7$, and further active amplification continues to improve between p14 to p20 (Song, McGee et al. 2008). In this study, the single fiber tuning properties will be directly characterized in p14-15 and p20-21 mice.

(3) Discharge rate: ANFs in kitten reach maximum discharge rates to tone bursts at CF at around p20 (Walsh and McGee 1987), during which period the wave I amplitude and latency of auditory brainstem response (ABR) further develop to mature level (Walsh, McGee et al. 1986, Walsh, McGee et al. 1986). A similar postnatal development of ABR responses happens in mice (Song, McGee et al. 2006). The ABR wave I amplitude is thought to reflect synchronous ANF responses (Melcher, Guinan et al. 1996, Melcher and Kiang 1996, Melcher, Knudson et al. 1996). Another purpose of this study is to characterize suprathreshold ANF discharge activity underlying the gross ABR wave I.

(4) Rate-level function: The shape of rate-level functions can be affected by several factors, for example, OHCs and IHCs function (Heinz and Young 2004). During early postnatal development, the functional state of cochlea gradually reaches a mature level. By comparing rate-level functions of ANFs from two different age groups (p1415 and p20-21), essential information might be obtained about the main development events which shape the rate-level function.

\subsection{Bassoon and synaptic ribbon}

\subsubsection{Synaptic ribbon and transmitter release at ribbon synapses}

Sensory hair cell synapses are specialized for fast and sustained neurotransmitter release to precisely encode time-varying sensory stimuli. Their morphological hallmark is the presence of synaptic ribbon, an ovoid electron-dense structure tethering vesicles around it (Fuchs, Glowatzki et al. 2003, Nouvian, Beutner et al. 2006). Similar ribbon structures can also be found in electroreceptors, retinal photoreceptors and bipolar cells, which together are sensory neurons encoding information by means of graded membrane potentials (Dieck and Brandstätter 2006). In mature mouse IHCs, synaptic ribbons are ovoid in shape and anchored several nanometers away from the presynaptic AZs by a 
single arcuate density, tethering 100 to 200 synaptic vesicles by rows of filament-like structures (Sobkowicz, Rose et al. 1982, Khimich, Nouvian et al. 2005).

The molecular composition of synaptic ribbon is not entirely clear (review in Pangrsic et al, TINS 2012). Recent biochemical studies have identified several main protein components of the synaptic ribbon. First, there is RIBEYE protein, which is an alternative splicing variant of the transcriptional suppressor, C-terminal binding protein 2(CtBP2) (Piatigorsky 2001, Khimich, Nouvian et al. 2005, Uthaiah and Hudspeth 2010). Second, there is RIM protein, which is a synaptic protein also present at conventional synapses where it interacts with a rab3, a GTPase expressed on synaptic vesicles (Schmitz 2009, Uthaiah and Hudspeth 2010). Third, KIF3A, a kinesin motor protein has also been identified (Muresan, Lyass et al. 1999). Bassoon and Piccolo, which are presynaptic cytometric proteins also found at conventional synapses, are expressed at ribbon synapses (Khimich, Nouvian et al. 2005). The expression of Bassoon and Piccolo may vary in different cell types. At bipolar cell ribbon synapses, only Piccolo is expressed, while both proteins are expressed at photoreceptor ribbon synapses (Brandstatter, Fletcher et al. 1999, Dick, Hack et al. 2001).

Currently, several hypotheses exist about the function of synaptic ribbons. Given the morphological observation of large number of synaptic vesicles tethered around the structure, it is tempting to speculate that synaptic ribbon acts like "conveyor belt" to supply vesicles. A role of synaptic ribbon in rapid replenishment of vesicles during sustained neurotransmitter release has been reported in several studies (Parsons, Lenzi et al. 1994, von Gersdorff, Vardi et al. 1996, Frank, Rutherford et al. 2010, Snellman, Mehta et al. 2011). Another hypothesis suggests synaptic ribbons are important for establishing and stabilizing a large number of vesicle release sites, $\mathrm{Ca}^{2+}$ channels at the active zone thereby a large readily releasable pool of vesicles (RRP) (Khimich, Nouvian et al. 2005, Frank, Rutherford et al. 2010). The third hypothesis suggests a role of synaptic ribbons in coordinating multi-vesicular release. Experimental evidences supporting multivesicular release have been reported for inner ear and retinal ribbon synapses (Glowatzki and Fuchs 2002, Singer, Lassova et al. 2004, Singer 2007). 


\subsubsection{Presynaptic scaffold protein Bassoon and mouse mutants}

Bassoon is a large $420 \mathrm{kDa}$ scaffold protein. It is a zinc-finger CAG/glutamine-repeat protein located at presynaptic active zones of both excitatory and inhibitory synapses (tom Dieck, Sanmarti-Vila et al. 1998, Richter, Langnaese et al. 1999). Bassoon is structurally similar to another presynaptic zinc finger protein Piccolo (Fenster, Chung et al. 2000). Both proteins appear at nascent synapses during early stage of synaptogenesis and therefore a role in synapse formation is suggested (Friedman, Bresler et al. 2000, Zhai, Olias et al. 2000). At photoreceptor ribbon synapses, Piccolo and Bassoon might have different roles, as Bassoon is found at the base of the ribbon, while Piccolo is concentrated at the apical region (Dick, Hack et al. 2001).

It has been shown that Bassoon can interact with RIBEYE (tom Dieck, Altrock et al. 2005). Using mutant mice lacking a central domain of Bassoon $\left(\mathrm{Bsn}^{\Delta \mathrm{Ex} 4 / 5}\right.$, partial deletion of exon 4 and 5 of bassoon gene) a role of Bassoon in the anchorage of ribbon to the active zones of photoreceptors (Dick, tom Dieck et al. 2003) and inner hair cells (Khimich, Nouvian et al. 2005) has been suggested. In addition, mutant ribbon synapses show aberrant clustering of voltage-gated calcium channels and a reduced number of readily releasable vesicles (Khimich, Nouvian et al. 2005, Frank, Rutherford et al. 2010), leading to reduced spike rates of auditory nerve firing (Buran, Strenzke et al. 2010, Frank, Rutherford et al. 2010).

IHCs of $\mathrm{Bsn}^{\Delta \mathrm{Ex} 4 / 5}$ mutant mice (Altrock, tom Dieck et al. 2003) show severe synaptic ribbon loss (Altrock, tom Dieck et al. 2003, Khimich, Nouvian et al. 2005). To exclude a possible function the remaining fragment in $\mathrm{Bsn}^{\Delta \mathrm{Ex} 4 / 5}$, a newly generated bassoon genetrap mutation was used. Interestingly, IHCs of another newly generated bassoon genetrap mutation $\left(\mathrm{Bsn}^{\mathrm{gt}}\right)$ mice line show a higher fraction of preserved synaptic ribbons compared to that of $\mathrm{Bsn}^{\Delta \mathrm{Ex} 4 / 5} \mathrm{IHCs}$. The different ribbon anchorage phenotypes provided new opportunities for differentiating bassoon/ribbon effect on synaptic physiology.

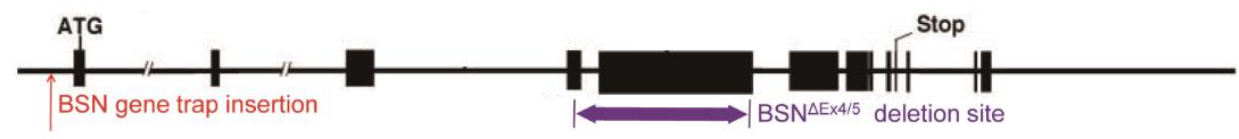


Figure 1.3: Illustration of the Bassoon gene and two available mutations.

$\mathrm{BSN}^{\Delta \mathrm{Ex} 4 / 5}$ (purple): the main coding exons 4 and 5 of the Bassoon gene are knocked out.

$\mathrm{BSN}^{\mathrm{gt}}$ (red): a transcriptional terminal sequence (gene trap vector) is inserted before the first exon to block transcription.

\subsubsection{Purpose of the study using Bassoon mutants}

The study started with the hypothesis that a higher fraction of remaining anchored synaptic ribbons in Bsn ${ }^{\text {gt }}$ IHCs might cause a milder phenotype than observed in ANF of $\mathrm{Bsn}^{\Delta \mathrm{Ex} 4 / 5}$ hair cells, which have a near-complete loss of ribbons from synapses.

In collaboration with other members of the InnerEarLab, the study intended to address the following issues:

1. To quantitatively compare ribbon synapses anchorage phenotypes in two mutants.

2. To explore one possible explanation for the ribbon anchorage phenotype: leaky expression of bassoon in IHCs.

3. To address a potential loose anchorage of remaining synaptic ribbons to the presynaptic active zones by analyzing 3D confocal images.

4. To quantitatively analyze the effect on presynaptic $\mathrm{Ca}^{2+}$ channel and postsynaptic GluA2 receptor clustering.

5. To thoroughly compare the in vitro cell physiology and sound encoding phenotypes in ANFs of both mutants.

Collective results from this study demonstrate that IHC synapses in Bsn mutants have an abnormal morphology and function, irrespective of the presence or absence of a synaptic ribbon. The remaining ribbons in $\mathrm{Bsn}^{\mathrm{gt}}$ IHCs were most likely not fully functional (presumably due to looser anchorage), or other perturbations of the synaptic ultrastructure (e.g. abnormal calcium channel clustering) dominate the sound encoding deficits. 


\subsection{Black swiss mice and hearing deficits}

\subsubsection{BLSW mice and hearing impairment phenotypes}

Dysfunction or loss of hair cells of the organ of Corti or cochlear nerves results in sensorineural hearing loss (SNHL). Deficits in hearing can lead to severe impairment of quality of life. About half of the congenital deafness cases is of genetic origin and genetic predisposition is one of the risk factors in susceptibility to SNHL later in life. Nearly 100 genes have been reported with mutations to be involved in hearing impairment in mouse (Willott 2001). The similarity of the mouse inner ear anatomy, hereditary pathologies and genetics to that of human, makes mice an excellent model to study genes underlying human hearing impairment and hearing loss (Steel 1991).

Besides using forward genetic approaches like targeted mutational analysis by genetic engineering of known genes in mice to understand the molecular mechanisms underlying sound encoding, certain mouse strains show spontaneously arisen hearing defects. Studies of those mice have helped uncovering the pathological process underlying auditory system dysfunctions (Steel 1995).

The sound encoding defect underlying the hereditary hearing loss phenotypes in Black swiss (BLSW) mice is unknown. BLSW mice exhibited retinal degeneration (Clapcote, Lazar et al. 2005), and early-onset, progressive SNHL (Drayton and Noben-Trauth 2006, Charizopoulou, Lelli et al. 2011). BLSW mice have been shown to have high susceptibility to audiogenic seizures, characterized by wild running, loss of righting reflex, and tonic and clonic convulsions (Misawa, Sherr et al. 2002). The susceptibility to audiogenic seizures in BLSW mice peaks at p21 and nearly disappeared by adulthood (Misawa, Sherr et al. 2002, Charizopoulou, Lelli et al. 2011). Two quantitative trait loci (QTL) have been identified underlying hearing loss in BLSW mice: age-related hearing loss 5 (ahl5) and ahl6, which account for $61 \%$ and $32 \%$ of the variation observed (Drayton and Noben-Trauth 2006). Moreover, the juvenile audiogenic monogenic seizure 1 (jams1) locus is found to be underlying the audiogenic seizures (Misawa, Sherr et al. 2002). Genetic mapping suggested adh5 and jams1 phenotypes have a common underlying allele. Indeed a missense mutation affecting the PDZ domain of GIPC3 has been identified as the cause underlying SNHL (adhl5) and audiogenic seizure (jams1) in BLSW mice (Charizopoulou, Lelli et al. 2011). In human, a frameshift and six missense mutations in 
GIPC3 underlie nonsyndromic hearing loss DFNB72 (Rehman, Gul et al. 2011), and a missense mutation in W98-042 family leads to early onset bilateral SNHL DFNB95 (Charizopoulou, Lelli et al. 2011).

GIPC3 is a small $\sim 40 \mathrm{kDa}$ protein, which belongs to the GIPC family. Three members have been identified: GIPC1, GIPC2 and GIPC3, which show over 50\% total-amino-acid identity between each other (Katoh 2002). All three members have a central PDZ domain and two GIPC homology domains. The PDZ domain of the GIPC protein family has been shown to interact with Frizzled-3 (FZD3) class of WNT receptor, insulin-like growth factor-I (IGF1) receptor, receptor tyrosine kinase TrkA, TGF-beta type III receptor (TGFbeta RIII), integrin alpha6A (ITGA6), transmembrane glycoprotein TPBG and regulator of G-protein signaling 19 (Rgs19) (Katoh 2002), implicating a role as an adaptor protein in cell signaling pathways. GIPC3 is expressed in IHCs, OHCs and spiral ganglion neurons. Interestingly, GIPC3 has a similar localization in hair cells as Vglut3 and Myosin VI, both of which can regulate synaptic transmission at the IHC ribbon synapse (Charizopoulou, Lelli et al. 2011).

Charizopoulou et al. (2011) have characterized the SNLH phenotypes in BLSW mice at the systems, morphology and cell physiology level. Their findings can be summarized as follows: (1) In the cochlea of BLSW mice, the expression level of GIPC3 is reduced by $69 \%$ at protein level. (2) Analysis of the morphology of organs of Corti of BLSW mice have revealed that both OHCs and IHCs have abnormal hair bundle organization as early as P3 and P5. (3) Both OHCs and IHCs have a reduction of mechanotransduction current at P12. In addition, IHCs of BLSW mice show smaller potassium currents at P13 compared with control of similar age, which was attributed to impaired postnatal development. (4) At 4-weeks of age, BLSW mice have elevated hearing threshold and reduced DPOAE amplitude. The ABR wave I amplitude at P14 is significantly larger compared to that of control. The larger wave I amplitude at P14 was correlated with susceptibility to audiogenic seizures early after the onset of hearing. (5) Late-onset degeneration of the organ of Corti and severe loss of spiral ganglion neurons were observed, especially at base and the mid-apical region. 


\subsubsection{Purpose of the study using BLSW mice}

One hypothesis built upon the results of the above described studies is that the larger wave I amplitude, which represents the summed activity of the ANFs, is responsible for seizure susceptibility at P14 in BLSW mice (Charizopoulou, Lelli et al. 2011). This might result from the reduced IHCs $\mathrm{K}^{+}$currents, which allows greater depolarization of IHCs receptor potential during stimulus. This may lead to enhanced $\mathrm{Ca}^{2+}$ dependent neurotransmitter release, and subsequently elevated synchronized activity of ANFs.

Recording from ANFs in BLSW mice allows a direct assessment of potential abnormally high or synchronized activation of SGN. In addition, several predictions can be made given the above results: (1) Reduced DPOAE suggests a problem in mechanical amplification. Without this amplification, the frequency selectivity of single ANF would be degraded. (2) The compressive nonlinearity provided by active amplification of OHCs allows ANFs to encode broad stimulus intensity range. An impaired active amplification can result in a steeper growth of ANF rate-level functions. (3) Lack of IHCs $\mathrm{K}^{+}$currents might lead to a depolarized receptor potential at rest, which may enhance neurotransmitter at rest, producing a higher spontaneous activity of ANFs. In addition, spontaneous and evoked spike rates may be altered by changes in presynaptic physiology, which was not addressed in (Charizopoulou, Lelli et al. 2011). (4) Abnormal ANF firing patterns may underlie the audiogenic seizures.

This study was intended to address the following questions to better understand the sound encoding deficit in BLSW mice:

1. To characterize the changes in ANFs response properties in BLSW mice between 23 weeks, the period during the period of which the ABR wave I amplitude decreased and to explore how those two measurement can be together reflect the pathology of the SNHL in BLSW mice.

2. To study whether the ANFs responses are consistent with predictions given by the known sites of lesion in the inner ear. 


\subsection{Cochlear implants in restoring the sense of hearing}

When hearing impairment happens, auditory prostheses can be used to restore lost auditory functions. Currently, two types of auditory prostheses are in common use. Conventional hearing aids amplify sounds to overcome threshold increases. Cochlear implants (CIs) are provided to patients who are completely deaf because of loss of inner hair cells. CIs bypass the damaged cochlea and stimulate remaining afferent nerve fibers directly by electrical currents to provide patient with environmental sound and, in most cases, speech comprehension. CIs are now recognized as effective auditory prostheses for many people suffering from profound hearing impairment. For patients whose ANFs does not function, auditory brainstem implants (ABIs) are used instead. However, ABIs are less frequently applied to patients due to requirement of brain surgery and the sound perception provided by ABIs is less effective (Møller 2006).

Current CIs employ several separate channels to stimulate ANFs at different tonotopic locations. The purpose of this is to map frequency components of incoming sound to the appropriate place on the tonotopic axis. There are several limitations of such scheme. First, it is difficult to surgically implant the electrode array to cover the whole tonotopic area due to the coiled structure of the cochlea. Therefore, the frequency range that the implanted electrode array stimulates is a subrange of what a normal cochlea uses. Second, currents coming from one monopolar electrode spreads within the cochlea and in addition to exciting afferent fibers nearby also stimulates additional frequencies which leads to reduced number of independent channels. Bipolar and multipolar stimulation has been developed to overcome such difficulties. However, the improvement of frequency resolution by multipolar stimulation comes at the expense of higher energy consumption. Third, the distance between the electrodes to afferent fibers further pose a physical limit of the number of channels and minimal effective electrode currents. To solve this problem requires direct contact between electrode and afferent fibers, which is surgically much more challenging.

One novel method to overcome difficulties of electrical stimulation is to use optical stimulation. It has recently been shown that mid-infrared (IR) lasers can evoke action potentials from ANFs (Littlefield, Vujanovic et al. 2010). Although the mechanisms of neural excitation by IR laser is not entirely clear, compared with electrical stimulation, 
IR stimulation have much smaller spatial activation pattern by selectively activating neurons along directly along the optical path. Optical stimulation could potentially improve the frequency resolution by providing more independent stimulation channels. Besides, a potential advantage of more independent stimulation channels, simultaneous excitation and inhibition of neighboring afferent fibers may better mimic nonlinear behavior of basilar membrane.

Recently developed optogenetic tools have made it possible to selectively excit and inhibit neural activities by blue and green light. Optogenetic stimulation of neurons is based on channelrhodopsins (ChRs), a subfamily of rhodopsins that function as lightgated ion channels. Originally they were found in algae where they are involved in phototaxis. ChR 1 and $\mathrm{ChR} 2$ are the first two types of proteins used as optogenetic tool in neuroscience. When expressed in neurons, a blue light illumination results in depolarizating photocurrents by inducing conformational changes. ChR2 has been widely used for targeted activation of neurons in vitro and in vivo. Towards a clinical application of optogenetic tools and to achieve higher light sensitivity and faster activation, the ChR2variant CatCh was developed (Kleinlogel, Feldbauer et al. 2011). Expression of ChR2 in retinal ganglion cells has been shown to be able to restore visual function in blind rats (Tomita, Sugano et al. 2010) and a mouse model of retinal degeneration (Lagali, Balya et al. 2008). This study is intended to explore the potential application of optogenetic stimulation of auditory afferent nerves expression of ChR2 by first characterization of light evoked activities.

\subsection{Aims and scopes of the studies}

To better understand how the cochlear mechanisms relevant to sound encoding in the ANF responses in this thesis, mouse models were used to study sound encoding in the mouse cochlea. The availability of genetic manipulation as well as certain strains carrying genes responsible for various types of SNHL pathology allow us to address questions about the molecular mechanisms underlying cochlea sound encoding, which otherwise could not be done in other widely used model mammals like cat, guinea pig, gerbil and chinchilla. 
In the first part of the thesis, the developmental changes of ANF responses during early postnatal development in mice will be characterized for the first time. In vitro experiments and immunohistochemistry have suggested a developmental change of presynaptic $\mathrm{Ca}^{2+}$ channels organization and signaling during the 2 to 3 postnatal week. The question whether ANF response properties change during the same development period will also be explored.

Inner hair cells are specialized for sustained transmission with high temporal precision, featuring the presence of an electron-dense structure, the synaptic ribbon. The availability of genetic manipulation methods in mice can help us identify the molecular mechanisms of sound encoding underlying normal or pathological conditions. As an example to further understand the role of synaptic mechanisms in the sound encoding, ANF sound encoding in Bassoon mouse mutants was characterized in the second part of the thesis. Previous results have demonstrated that in Bassoon mutants carrying a bassoon gene lacking the central region of exons 4 and $5\left(\mathrm{Bsn}^{\Delta \mathrm{Ex} 4 / 5}\right)$ show severe ribbon loss at presynaptic active zones and deficits in sound encoding. A newly generated Bsn genetrap mouse strain $\left(\mathrm{Bsn}^{\mathrm{gt}}\right)$ showed somehow milder ribbon loss phenotype compared to the previous mutant strain. By comparing these two mutants, we might be able to differentiate ribbon dependent functions. Experimental evidences supporting the notion of 'sick' loosely anchored synaptic ribbon in Bassoon mutants will be shown, which argue for the important role of Bassoon/synaptic ribbon in the organization and function of IHC presynaptic active zone and sound encoding.

Damage to the delicate sensory hair cells in the cochlea leads to SNHL. The mammalian inner ear of different species shares a common organization. It is thought that, except for different frequency range of hearing, the sensorineural function in mice is similar to that in human. Studying the SNHL in mice may provide us insight into human pathology. Therefore, black swiss mice strain (BLSW), which shows age dependent SNHL and audiogenic seizures, was used as another example of using single unit recording from ANFs to demystify the potential source of lesion in the mouse cochlea. BLSW mice carry a mutation in GIPC3 gene, which has been shown to be underlying human autosomal recessive deafness DFNB15 and DFNB95 (Charizopoulou, Lelli et al. 2011). By studying the ANF response properties in BLSW mice, it was found that one of the most likely deficiencies in this strain is mechanical amplification in the cochlea. In addition to the 
OHCs problem, there might also be inner hair cell changes. Both abnormal of OHCs and IHCs contribute to the auditory phenotypes in BLSW mice.

Having learned the important role of sensory hair cells in sound encoding in the mouse cochlea, what can be done if sensory hair cells are damaged that they can no longer faithfully transmit temporal information onto the ANFs? The current approach is to use cochlea implant to directly stimulate ANFs using electrical signals. This method has been successfully applied to human patients. Efforts have been made to enhance the hearing experience like music perception using cochlea implant. However, the number of useful channels which can be used using electrical signal is limited by the spread of current in the tissue. As a potential alternative stimulation method, optical instead of electrical stimulation might improve the number of stimulation channels. In the last part of the thesis, optical stimulation of mouse ANFs expressing Channelrhodpsin 2(ChR2) as well as its variant CatCh was used to characterize the evoke responses of ANFs/cochlear nucleus neurons.

Results from those studies as a whole demonstrate the power of single unit recordings from mice ANFs. These data are one step towards understanding the sound encoding in the mouse cochlea. In depth understanding of this process requires the integration of in vivo ANF recordings, in vitro cell physiology as well as computational modeling. By accumulating data using different experimental approaches and manipulations of different coding components in the mouse cochlea, future studies will help us depict a more comprehensive picture about this process. 


\section{Materials and Methods}

\subsection{Animals}

The following mouse lines were used in the studies covered in this thesis:

1) C57BL/6 mice aged between $\mathrm{p} 10$ and $\mathrm{p} 21$.

2) Black swiss mice (BLSW) aged between p14 and p25. BLSW mice were generated by outcrossing albino NIH swiss mice to C57BL/6N and then backcrossing to the NIH swiss line (Clapcote, Lazar et al. 2005). The early-onset hearing loss in BLSW mice has been a model for progressive sensorineural hearing loss and the GIPC3 mutation has been found to underlie the hearing phenotype (Charizopoulou, Lelli et al. 2011). C57BL/6 mice served as controls.

3) Bassoon mutants and wildtype littermates aged between 5-11 weeks with a targeted deletion of exons 4 and 5 of the bassoon gene $\left(\mathrm{Bsn}^{\Delta \mathrm{Ex} 4 / 5}\right.$ ) (Altrock, tom Dieck et al. 2003). Mice were bred from parents of inbred C57BL/6 and 129/Sv background heterozygous.

4) Bassoon gene-trap mutants and wildtype littermates aged between 5-11 ( $\mathrm{Bsn}^{\text {gt }}$; derived from Omnibank ES cell line OST486029; Lexicon Pharmaceuticals) (Frank, Rutherford et al. 2010, Hallermann, Fejtova et al. 2010). The genetic background is uncharacterized but derived from the same C57BL/6 x 129/Sv strain as Bsn ${ }^{\Delta \mathrm{Ex} 4 / 5}$.

5) 6-12 week-old transgenic ChR2 mice (Arenkiel, Peca et al. 2007).

6) 6-12 week-old mice from CD1 background which had undergone transuterine otocyst injections (Brigande, Gubbels et al. 2009, Reisinger, Bresee et al. 2011) of adenoassociated-virus 6 (AAV6) to express the ChR2-variant CatCh (Kleinlogel, Feldbauer et al. 2011)in their SGNs.

All experiments complied with national animal care guidelines and were approved by the University of Göttingen Board for animal welfare and the animal welfare office of the state of Lower Saxony. 


\subsection{D confocal image analysis}

To explore the effect of Bassoon disruption on ribbon anchorage and Cav1.3 clustering in $\mathrm{Bsn}^{\mathrm{gt}}$ mice, quantitative 3D image analysis was performed using immunhistochemistry and confocal microscopy of organs of Corti stained for goat anti-CtBP2 (1:150; Santa Cruz Biotechnology), mouse anti-GluA2 (1:75; Millipore), Rabbit anti-CaV1.3 (1:75; Alomone Labs) (preparation, staining and imaging were carried out by Dr. Mark. A. Rutherford as described in (Frank, Rutherford et al. 2010)). The image analysis routines were based on Matlab (MathWorks, Inc.; programmed by Mr. Gerhard Hoch). The locations of synapses in confocal image stacks were defined as the centers of mass of fluorescent spots after thresholding by a subjective intensity criterion for GluA2 channel clusters. Locations of $\mathrm{CtBP} 2$ and $\mathrm{Cav} 1.3$ clusters were defined in the same manner. The average voxel intensity in the entire synaptic region excluding voxels that exceeded the threshold value was defined as background and subtracted from each image stack. Gaussian functions were fitted in $\mathrm{X}, \mathrm{Y}$ and $\mathrm{Z}$ dimensions to locate the voxel of center of mass of each clusters and also the distance to the centers of mass of the nearest neighboring cluster in the other two channels within a $500 \mathrm{~nm}$ range. Immunofluorescence intensity was quantified by integrating the voxel intensity values within a region of interest centered on the voxel where the center of mass of each GluA2positive was located. The size of the region of interest was $9 \times 9 \times 7$ voxels in $\mathrm{X}, \mathrm{Y}$ and $\mathrm{Z}$ direction respectively, corresponding to a volume of $\sim 0.5 \times 0.5 \times 3.0 \mu \mathrm{m}$.

\subsection{Real time PCR}

Real time PCR was performed in order to study the expression of bassoon on the mRNA level in $\mathrm{BSN}^{\mathrm{gt}}$ mice. Total RNA was isolated from organ of Corti preparations of 5-weekold mice using TRIzol (Invitrogen). cDNA were obtained by reverse transcription using random hexamers as primers. Real time PCR was done using ABI Prism 7900 Sequence Detection System (Applied Biosystems). In brief, cDNAs for Bsn, otoferlin and TBP (TATA-binding protein as a housekeeping gene) were selectively amplified using the Taqman Gene Expression Assays Mm01330351_mH, Mm00453306_m1, and Mm00446973_m1, respectively (Applied Biosystems) in separate reactions (20 $\mu 1$ volume) following the manufacture's protocol. Relative amounts of bassoon mRNAs 
were normalized the expression level of otoferlin using the comparative $2^{-\Delta \Delta \mathrm{Ct}}$ method (Applied Biosystems).

\subsection{ABR measurement}

Animals were anesthetized with ketamine (125 mg/kg, i.p.) and xylazine ( $2.5 \mathrm{mg} / \mathrm{kg}$, i.p.). Three subcutaneous needle electrodes were used for recording, one underneath the pinna $(+)$, one on the vertex (-) and one on the back near the legs as a reference. Stimulus generation and presentation, as well as data acquisition and processing were controlled by TDT III System (Tucker-Davis-Technologies). Sound pressure levels are provided in dB SPL RMS (tonal stimuli) or dB SPL peak equivalent (PE, clicks) and calibrated using a 1/4" Brüel and Kjaer microphone (D 4039, Brüel and Kjaer GmbH). Tone burst (10 ms plateau, $1 \mathrm{~ms} \cos ^{2}$ rise/fall) or clicks of $0.03 \mathrm{~ms}$ were presented at $20 \mathrm{~Hz}$ in the free field ipsilaterally using a JBL 2402 speaker (JBL GmbH\&Co.). A custom-designed amplifier was used to amplify the difference potential between the mastoid and vertex needles, sampling at $50 \mathrm{kHz}$ for $20 \mathrm{~ms}$ and filtered offline $(300-3000 \mathrm{~Hz}$ for aABR, 1-10000 Hz for $\mathrm{OABR}$ ). Hearing threshold was determined by visual inspection as the lowest stimulus intensity that evoked a reproducible response waveform.

\subsection{Single unit recording from mouse auditory nerve fibers}

\subsubsection{Surgical approach}

Single unit recordings were applied to study the sound encoding in individual ANFs. Animals were anesthetized with combination of xylazine $(5 \mu \mathrm{g} / \mathrm{g}$, i.p. $)$ urethane $(1.3 \mu \mathrm{g} / \mathrm{g}$, i.p.) and buprenorphine $(0.1 \mu \mathrm{g} / \mathrm{g}$, i.p. $)$. Animal temperature was maintained at $36.5{ }^{\circ} \mathrm{C}$ using a custom-designed heating pad in a sound proof chamber with ambient temperature of $33{ }^{\circ} \mathrm{C}$. The surgery and recording procedures were performed as described (Taberner and Liberman 2005, Strenzke, Chanda et al. 2009). The animal was tracheostomized and the cartilaginous ear canals were removed before the animal was placed in a custom designed stereotactic apparatus. The contralateral ear canal was plugged with moist cotton. The cochlear nucleus was exposed by an occipital approach with partial removal of the 
cerebellum. Glass microelectrodes filled with $2 \mathrm{M} \mathrm{NaCl}$ and $1 \%$ methylene blue were advanced in $4 \mu \mathrm{m}$ steps using an Inchworm micromanipulator (EXFO Burleigh) during noise burst stimulation, targeting the area of the ANFs' entry zone into the cochlear nucleus. The posterior region of the anteroventral cochlear nucleus (AVCN), where spherical bushy cells with similar responding pattern as ANFs are mainly located, was avoided during targeting.

Cochleostomy was performed on mice expressing either ChR2 or ChR2 variant CatCh to allow direct laser fiber stimulation of ANFs. A retroauricular approach was used to reach the left bulla. The cochlea was exposed after opening the bulla. In ChR2-mice, a small cochleostomy (diameter: $\sim 500 \mu \mathrm{m}$ ) was made by gentle shaving of the bony cochlear capsule. As CatCh was mainly expressed in the basal turn, the optical fiber was inserted into the cochlea through the round window when working on those transuterine injected mice. A $250 \mu \mathrm{m}$ optical fiber coupled to a $473 \mathrm{~nm}$ laser (MBL473, $50 \mathrm{~mW}$ DPSS, Changchun, China) was then inserted into the cochleostomy and round window, respectively. Irradiance was calibrated with a laser power meter (Laser check, Coherent Inc.) before each experiment.

\subsubsection{ANF identification}

Sound-responsive neurons were identified by $50 \mathrm{~ms}$ noise bursts presented at $90 \mathrm{~dB}$. ANFs were distinguished from primary cochlear nucleus units based on the electrode position (interior auditory canal, deeper than $1 \mathrm{~mm}$ below the surface of cochlear nucleus) and their primary-like response characteristics to suprathreshold ( $30 \mathrm{~dB}$ above threshold) $50 \mathrm{~ms}$ tone bursts presented at the characteristic frequency of the ANF. A coefficient of variation of inter-spike intervals of adapted responses $>0.5$ confirmed the irregular firing pattern that is typically observed in ANFs (Taberner and Liberman 2005). Following the experiment, the cochlear nucleus was aspirated to expose the ANF and interior auditory canal to verify that the electrode had been in the right position. Erroneous unit type classification was more likely to occur when firing rates were low (e.g. in Bassoon mutant mice), yielding less clear response patterns. 
In pre-hearing mice (p10-p12), the identification of ANFs based on acoustic evoked primary-like response was impossible because ANFs did not respond to maximum acoustic stimuli available at the stimulus rates used. In this case, stereotactic position of recording was always verified after the recording to make sure the correct targeting. The depth criterion (>1000 mm below the surface of cochlear nucleus) was also applied to these data.

\subsubsection{Acoustic system and signal processing}

The acoustic stimuli were generated digitally using TDT Systems 3 (Tucker-Davis Technologies). After sound pressure level adjustments by an attenuator (PA5, TuckerDavis Technologies), the sound was delivered by an ED-1 speaker (Tucker Davis Technologies) and a custom-designed close-field probe placed close to the opening of the left ear canal. Extracellular signals were amplified and bandpass filtered (300-3000 Hz) using an ELC-03XS amplifier (NPI Electronics). Upon detection of sound-evoked spiking activity on the connected oscilloscope (TDS2004B, Tektronix) and an audio monitor, signals were recorded using an analog-to-digital multifunction processor (RX6, Tucker-Davis Technologies) at sampling rate of $24414 \mathrm{~Hz}$. Stimulus presentation and recording were controlled by custom written MATLAB programs. For tuning curves, spike detection was performed online based on a manually set trigger, whereas all other data were analyzed offline using a spike detection routine modified from the Wave_Clus routines (Quiroga, Nadasdy et al. 2004).

\subsubsection{Stimulus and response measurements}

The spontaneous firing rate of each identified sound responsive unit was first measured in a $10 \mathrm{~s}$ interval without any stimuli. When the spontaneous rate was less than 10 or 2 $\mathrm{Hz}$, an additional 10 or $20 \mathrm{~s}$ recording interval was added, respectively. Then, an automatically adapting algorithm that systematically varied the frequencies and sound pressure levels of $15 \mathrm{~ms}$ tone bursts was employed to determine the characteristic frequency (CF) and best threshold for each unit. A sequential probability test (Wald 1947) with $\mathrm{p}=0.03$ was used to determine whether the resulting spike rate was equal to the 
spontaneous rate or to the threshold rate $(\mathrm{SR}+20 \mathrm{~Hz})$ or if the measurement had to be repeated to reach statistical significance. The initial step size of $16 \mathrm{~dB}$ was halved each time the threshold was crossed until a precision of $1 \mathrm{~dB}$ was reached. Similarly, the initial 1 octave frequency steps was halved for frequencies near the best frequency to determine the thresholds near the $\mathrm{CF}$ with $1 / 32$ octave precision. 50 msTone bursts at $\mathrm{CF}(2.5 \mathrm{~ms}$ $\cos ^{2}$ rise /fall, repetition rate at $8 \mathrm{~Hz}$ ) were used to characterize sound evoked responses.

Rate-level functions were measured using 25 tone bursts per level, ranging between 20 $\mathrm{dB}$ below and $50 \mathrm{~dB}$ above threshold. The sequence of stimulus levels was generated randomly in $5 \mathrm{~dB}$ steps. For each stimulus trial, the number of spikes was counted over a fixed window from the stimulus onset to the stimulus offset. Spike counts for each level were then averaged to generate a rate-level function. Rate-level functions were fitted using a five-parameter model (Sachs and Abbas 1974, Sachs, Winslow et al. 1989, Taberner and Liberman 2005). The input sound pressure " $P_{\text {in }}$ " first passes through a nonlinearity that represents the BM compressive nonlinearity (equation 1). $\theta_{\mathrm{I}}$ in equation 1 represent the point when $\mathrm{P}$ became compressed non-linear function of " $\mathrm{P}_{\text {in }}$ ". $\alpha$ is fixed at 0.27. The driving rate $R_{d}$ is expressed as a non-linear saturating function of BM output "P" (equation 2). $\theta_{\mathrm{E}}$ in equation 2 represents threshold of the saturating function. The exponent $\beta$ in equation 2 allows the saturation function have a steeper rate-level function. Finally, the total discharge rate is expressed as the sum of the stimulus-driven rate $R_{d}$ and a spontaneous rate $\left(\mathrm{R}_{\mathrm{sp}}\right)$. The fitting was done in Matlab using lsqcurvefit function, which solves nonlinear curve-fitting problem in least-square sense. Goodness of fit was assessed by computing root mean square error $(<20$ spikes/s). Dynamic range is defined as the range of sound pressure levels in which the rate-level function showed a rate increase between $10 \%$ and $90 \%$ of the difference between spontaneous and maximum rate (Figure 1.2). The calculation of dynamic ranges for ANFs were based on the model fitted ratelevel functions. For signal detecting analysis, the standard derivation of the rate across trials for each level was also computed and smoothed using a moving average filter.

$$
P=P_{\text {in }} \cdot\left(\frac{1}{1+\left(\frac{P_{i n}}{\theta_{I}}\right)^{2}}\right)^{\alpha}
$$




$$
R_{d}=R_{m} \cdot\left(\frac{1}{1+\left(\frac{\theta_{E}}{P}\right)^{\beta}}\right)
$$

$$
R_{t o t}=R_{s p}+R_{d}
$$

Recovery from adaptation was measured in the forward masking paradigm (Frank, Rutherford et al. 2010), in which a $100 \mathrm{~ms}$ tone burst (Masker, at CF, $30 \mathrm{~dB}$ above threshold) was followed by a $15 \mathrm{~ms}$ tone burst (Probe) presented at the same frequency and stimulus level as the masker. The masker and probe were separated by 3 or 4 randomly ordered intervals between 4 to $256 \mathrm{~ms}$. Stimulus trials were presented at a rate of $2 \mathrm{~Hz}$ rate and each interval was repeated 30 times.

To measure the ability of ANFs to encode amplitude modulated tones, transposed tones with CF carrier frequency which were amplitude modulated by a half wave rectified modulator sinusoid $(125 \mathrm{~Hz}, 250 \mathrm{~Hz}, 500 \mathrm{~Hz}$ and $1 \mathrm{kHz})$ were presented. For each modulation frequency, the time-varying amplitude of the carrier wave was varied in $5 \mathrm{~dB}$ steps and each trial lasted 2 s. The vector strength, or synchronization index (SI) (Goldberg and Brown 1969) was calculated as described. In brief, a postzero-crossing histogram was first generated using spike times to measure synchrony. Instead of using a synchrony noise floor, Rayleigh statistics (Mardia and Jupp 2000) was used to assess the statistical significance. Values of SI that failed to reach a criterion $p<0.001(r>13.5)$ were discarded. Rayleigh test values $>13.8(\mathrm{p}<0.01)$ indicated that the synchronization of neuronal activity reflected a sample of an oriented distribution that is not attributed to chance (Buunen and Rhode 1978).

\subsection{Data analysis and statistics}

Data analysis was performed using Matlab, Igor Pro (Wavemetrics), ImageJ software (http://rsb.info.nih.gov/ij/) and Graphpad (GraphPad Software, Inc.). For comparing two groups, unpaired, two-tailed t test were used if the samples passed normality test (JarqueBera test) and equal variance under F-test. In all other cases, the non-parametric unpaired, two-tailed Mann-Whitney-Wilcoxon rank test was used. For comparing multiple samples, 
one-way ANOVA followed by Dunn's tests were used. Means are expressed as mean \pm SEM, unless specified otherwise. Significance is shown as follows: $* p<0.05$, ** $\mathrm{p}<0.01, * * * \mathrm{p}<0.001$ and n.s. (not significant). 


\section{Results}

\subsection{Auditory nerve fiber responses during early postnatal development in mice}

\subsubsection{Developmental changes of spontaneous spiking activity of mouse ANFs before and after hearing onset}

No sound-evoked single-unit spiking activity was detected in p10-12 mice using noise or tone bursts up to $100 \mathrm{~dB}$ (Figure 3.1D). For this reason, putative ANF units were identified only by their stereotactic positions of the recording electrode in p10-12 mice, which was verified at the end of each experiment. The erroneous inclusion of cochlear nucleus units can nonetheless not be completely ruled out. Figure 3.1A shows spontaneous burst activity of one representative putative ANF. The spikes were clustered in "mini-burst", which were separated from each other by approximately $120 \mathrm{~ms}$. Minibursts were clustered into "maxi-bursts" that could last for $>1 \mathrm{~ms}$ and were separated from each other by intervals of several seconds (Figure 3.1A-B). In contrast, the interspike interval distribution in p14-15 and p20-21 mice can typically be described as Poisson process with a dead interval, with a coefficient of variation of approximately 1 (Kiang 1965). However, the multimodal ISI distribution in p10 mice was reflected in the increase of a ISI CV greater than $1(2.2 \pm 0.2, \mathrm{n}=15)$, which was significantly larger than that of p14-p15 or p20-21 ANFs (Figure 3.1C, 1.0 $\pm 0.1, \mathrm{p}<0.001, \mathrm{n}=10$ or $0.9 \pm 0.1$, $\mathrm{p}<0.0001, \mathrm{n}=11$; Kruskal-Wallis One Way ANOVA with post hoc Dunn's multiple comparison test). 


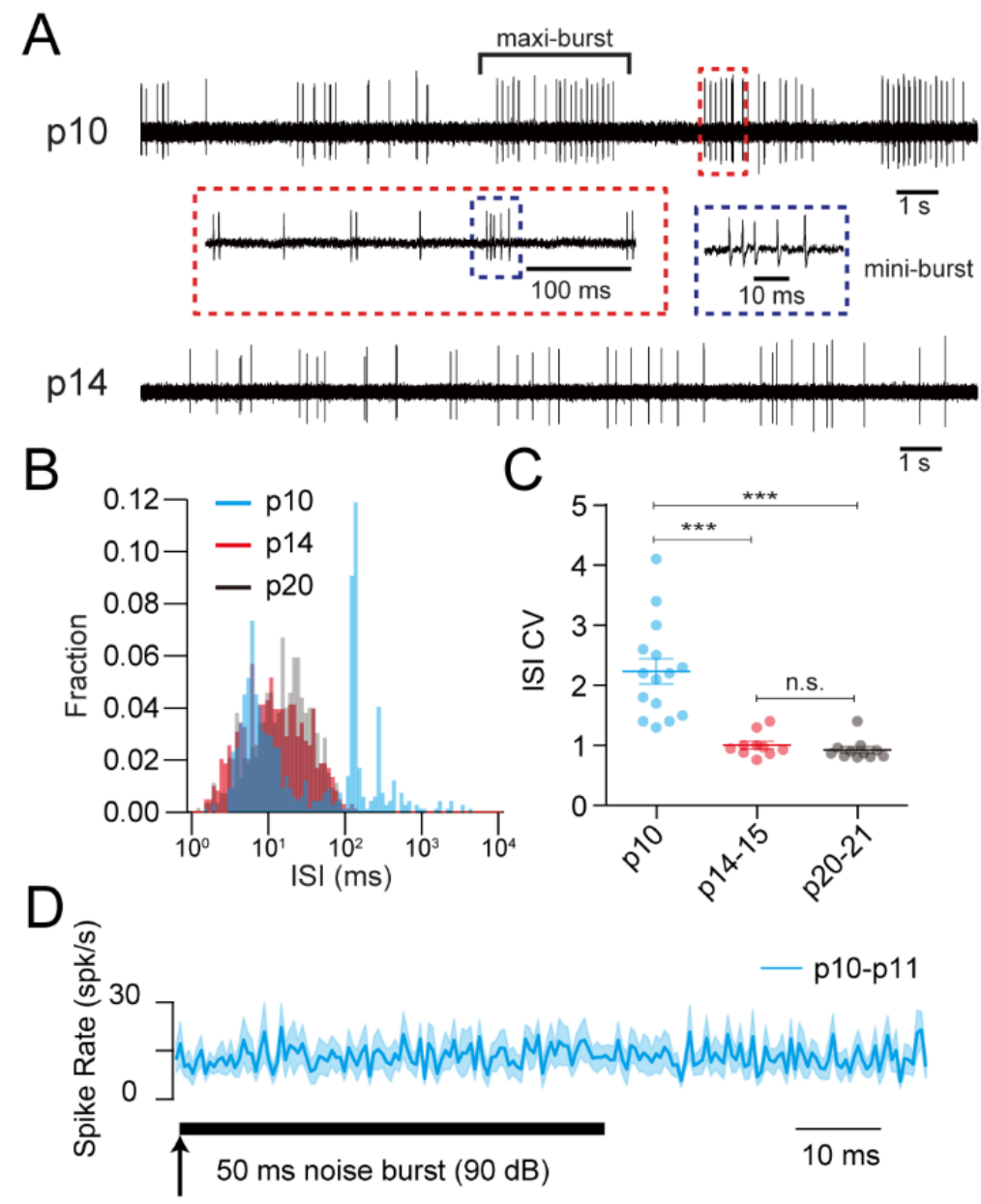

Figure 3.1: Putative ANF firing properties before hearing onset. (A) Example traces of spontaneous spiking activity of a p10 (top) and a p14 (bottom) ANF. The spontaneous activity in p10 ANFs was characterized by the presence of bursts of activity (maxi-burst) containing clusters of spikes (mini-bursts). (B) Inter-spike interval (ISI) histogram of the spontaneous activity of representative ANFs in three age groups (blue: p10; red: p14; black: p20). The multiple peaks in p10 include the interspike interval within a mini-burst ( 10 ms), the inter-mini-burst interval ( $120 \mathrm{~ms})$ and longer inter-maxi-burst intervals. (C) Coefficients of variation of ISI for ANFs in three development age groups. A CV around 1 is typical for a Poisson process. A higher CV is related to more regular bursting activity. The mean ISI CV for p10 ANFs $(n=15)$ was significantly larger than that for $\mathrm{p} 14-15$ or $\mathrm{p} 20-21$ ANFs $(\mathrm{p}<0.001, \mathrm{n}=10$ or $\mathrm{p}<0.0001$, $\mathrm{n}=11$ ). (D) Average post stimulus time histogram of $\mathrm{p} 10$ and $\mathrm{p} 11$ ANFs in response to $50 \mathrm{~ms}$ noise bursts at $90 \mathrm{~dB}$. No sound evoked responses were observed at this sound level at a stimulus rate of $8 \mathrm{~Hz}(\mathrm{n}=14)$.

In addition, when comparing the spontaneous rates of fibers recorded before and after the onset of hearing, the spontaneous discharge rate (averaged over the entire sampling period and including bursting activity) was significantly lower at p10-11 (Figure 3.2, 
p10-11, $11.6 \pm 2.0 \mathrm{~Hz}, \mathrm{n}=40$ vs. $\mathrm{p} 14-21,23.5 \pm 4.0, \mathrm{n}=24, \mathrm{p}<0.01$, Mann-Whitney test) while there was no statistic significant changes of spontaneous rate from p14-15 to p20$21(23.9 \pm 6.3 \mathrm{~Hz}, \mathrm{n}=12$ vs. p20-21, 23.5 $\pm 5.0 \mathrm{~Hz}, \mathrm{n}=12$, Mann-Whitney test, n.s.).

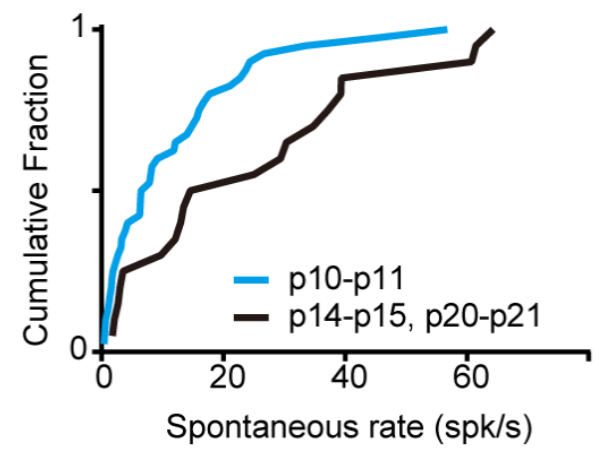

Figure 3.2: Cumulative distribution of spontaneous discharge rate of fibers before and after the onset of hearing. Note the right shift of distribution indicate the emergence of high spontaneous rate ANFs after the onset of hearing.

\subsubsection{Maturation of frequency selectivity after hearing onset}

After the onset of hearing, sound evoked responses of ANFs undergo further developmental refinement. The ANFs respond selectively to certain ranges of frequencies of sinusoidal stimuli that correspond to the cochlear tonotopic location of the inner hair cell they innervate. Thus, each ANF is tuned to a particular frequency, which is referred to as "characteristic frequency" (CF). The threshold was defined as the intensity of the stimulus level required to evoke a spike rate exceeding spontaneous rate by $20 \mathrm{~Hz}$. A tuning curve can be obtained by plotting threshold as a function of stimulus frequency. Figure 3.3A shows representative ANF tuning curves of the two age groups. P14-15 ANFs had tuning curve shapes resembling those of p20-21 ANFs. However, the ANF threshold at the CF further decreased as ANFs matured from p14-15 to p20-21 (Figure 3.3B, 8-16 kHz, $\mathrm{p}<0.01, \mathrm{n}=5$ for $\mathrm{p} 14-15, \mathrm{n}=7$ for $\mathrm{p} 20-21 ; 16-32 \mathrm{kHz}, \mathrm{p}<0.05, \mathrm{n}=6$ for p14-15, n=4 for p20-21, Mann-Whitney test). Sharpness of tuning was quantified for each tuning curve by calculating the $\mathrm{Q} 10 \mathrm{~dB}$, which is the $\mathrm{CF}$ of the fiber divided by the bandwidth of the tuning curve at $10 \mathrm{~dB}$ above the $\mathrm{CF}$ threshold. Because the Q10 $\mathrm{dB}$ is a function of CF in cat ANF (Kiang 1965), fibers were binned into three octave groups 
according to their CFs $(4-8 \mathrm{kHz}, 8-16 \mathrm{kHz}$ and $16-32 \mathrm{kHz})$. The frequency tuning of ANFs in p20-21 mice was significantly sharper compared to p14-p15 (Figure 3.3C, 8-16 kHz, $\mathrm{p}<0.01, \mathrm{n}=5$ for $\mathrm{p} 14-15, \mathrm{n}=7$ for $\mathrm{p} 20-21 ; 16-32 \mathrm{kHz}, \mathrm{p}<0.05, \mathrm{n}=6$ for $\mathrm{p} 14-15, \mathrm{n}=4$ for $\mathrm{p} 20$ 21, Mann-Whitney test, statistical testing was not done for the $4-8 \mathrm{kHz}$ group because of insufficient sample size).
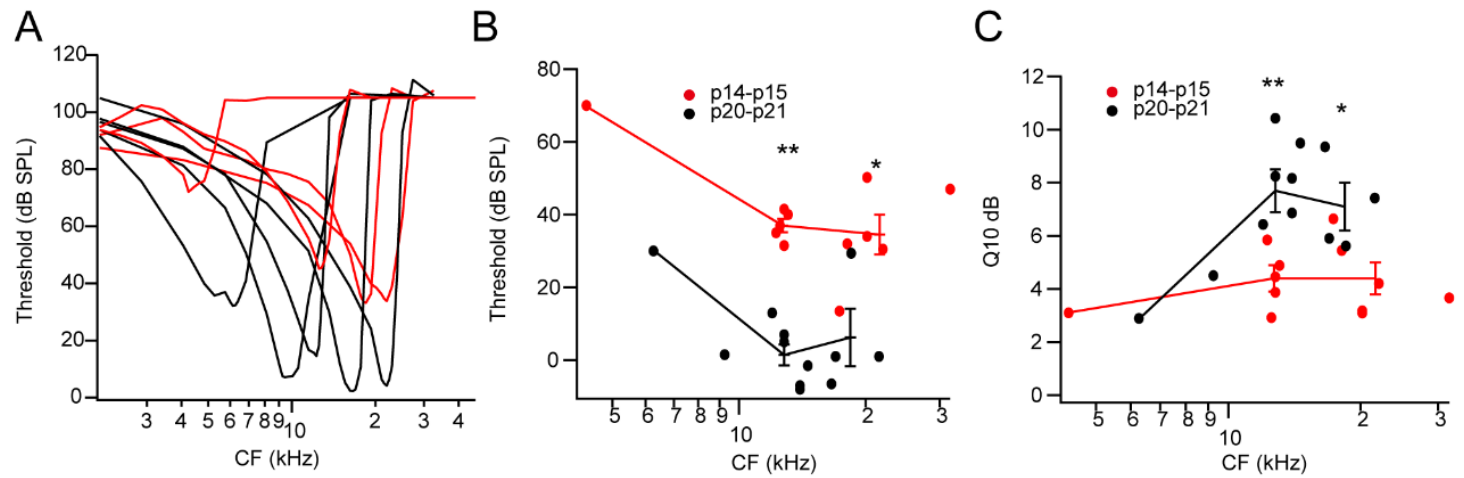

Figure 3.3: The hearing thresholds decreased and the sharpness of tuning increased during early postnatal development after onset of hearing. (A) Representative tuning curves of p14-15 (red) and p20-21 (black) ANFs. (B) The threshold at characteristic frequency for each fiber decreased from p14-15 to p20-21. (C) Sharpness of tuning, measured as Q10 dB, improved after onset of hearing from p14-15 to p20-21 ANFs.

\subsubsection{Maturation of response to pure tone bursts after hearing onset}

In response to $50 \mathrm{~ms}$ suprathreshold tone bursts presented $30 \mathrm{~dB}$ above threshold at $\mathrm{CF}$, ANFs fire trains of action potentials. The response latency for each fiber was quantified as median first spike latency. The response latencies in p14-15 were significantly longer than those at p20-21 (Figure 3.4B, p<0.01, $5.8 \pm 0.4 \mathrm{~ms} \mathrm{n}=12$ vs. $4.3 \pm 0.3 \mathrm{~ms}, \mathrm{n}=12$, Mann-Whitney test). The same tone burst stimuli was repeated 200 times, and a poststimulus-time histogram $(0.5 \mathrm{~ms}$ binwidth $)$ was constructed to display the time distribution of spike discharge time relative to the stimulus onset for each fiber. A typical ANF responded to tone bursts with a sharp onset response, which gradually adapted to a steady state rate. Figure 3.4A shows average PSTH of fibers aligned to their median first spike latency for the p14-15 and p20-21 age groups. There was a tendency of higher onset rates (firing rate in $1 \mathrm{~ms}$ after the response latency) in p20-21 ANFs ( $\mathrm{p}=0.06,391.7 \pm 36.2$ $\mathrm{Hz}, \mathrm{n}=12$ for $\mathrm{p} 20-21$ vs. $302.5 \pm 34.2, \mathrm{n}=12$ for $\mathrm{p} 14-15$, Mann-Whitney test), while the adapted rate (firing rate between 45 to $50 \mathrm{~ms}$ after response latency) was significantly 
larger in $\mathrm{p} 20-21$ ANFs $(\mathrm{p}<0.001,187.3 \pm 9.0 \mathrm{~Hz}, \mathrm{n}=12$ for $\mathrm{p} 20-21$ vs. $121.3 \pm 13.3 \mathrm{~Hz}$, $\mathrm{n}=12$ for $\mathrm{p} 14-15$, Mann-Whitney test). Adaptation was quantified by fitting a double exponential function to derive fast and slow adaptation components (Westerman and Smith 1984). Both the fast and slow adaptation time constants between the two groups were not significant different (fast: $\mathrm{p}=0.5,0.25 \pm 0.08 \mathrm{~ms}$ vs. $0.35 \pm 0.10 \mathrm{~ms}$; slow: $\mathrm{p}=0.8$, $11.3 \pm 1.2 \mathrm{~ms}$ vs. $11.9 \pm 2.0 \mathrm{~ms}, \mathrm{n}=12$ for $\mathrm{p} 20-21, \mathrm{n}=12$ for $\mathrm{p} 14-15$, Mann-Whitney test).

A
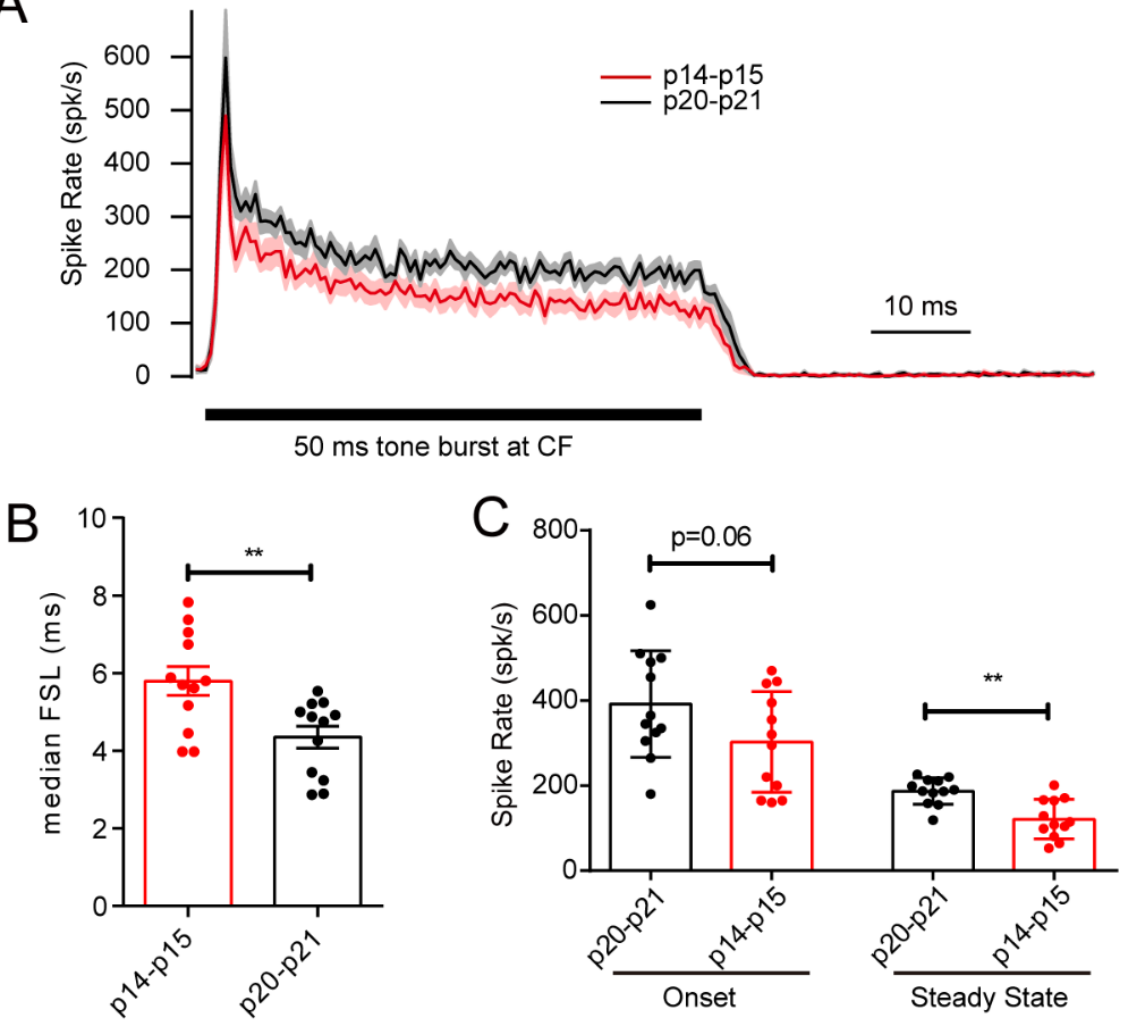

Figure 3.4: Development of ANF responses to suprathreshold tone bursts after the onset of hearing. (A) Average post-stimulus-time histogram (PSTH) of p14-15 and p20$21 \mathrm{ANFs}$ in response to $50 \mathrm{~ms} \mathrm{CF}$ tone bursts at $90 \mathrm{~dB}$. PSTHs were aligned to the bins of their respective median first spike latency before averaging. (B) p14-15 ANFs responded to tone bursts with longer latency. (C) The average steady state (adapted) rate was significantly higher in p20-21 ANFs compared to p14-15, while there was also a tendency for higher average onset rates.

\subsubsection{Development of rate-level function after hearing onset}

The discharge rate of ANF fibers increased monotonically with increasing stimulus levels (Sachs and Abbas 1974). This relationship was studied by measuring the rate-level function. A five parameter phenomenological model (Taberner and Liberman 2005) 
adapted from Sachs-Abbas model (Sachs, Winslow et al. 1989) was used to fit the ratelevel function to better approximate the dynamic ranges.

Figure 3.5A shows example rate-level functions of the two age groups. The improvement of hearing thresholds from p14-15 to p20-21 ANFs were reflected in the horizontal shift of rate-level functions. The dynamic range is defined as the range of sound pressure levels in which the rate-level function showed a rate increase between $10 \%$ and $90 \%$ of the difference between spontaneous and maximum rate of fitted rate-level function. No statistical significant difference between dynamic ranges was observed between the two age groups $(\mathrm{p}=0.65,12.7 \pm 4.4 \mathrm{~dB}, \mathrm{n}=11$ for $\mathrm{p} 20-21$ vs. $13.9 \pm 8.0 \mathrm{~dB}, \mathrm{n}=11$ for $\mathrm{p} 14-15)$. There was also no systematic correlation between spontaneous rates and dynamic ranges. However, ANFs with lower spontaneous discharge rates tended to have greater diversity in their dynamic ranges. Two p14-15 ANFs firing at spontaneous rates $<5 \mathrm{~Hz}$ showed non-saturating responses given the highest sound stimulus levels (Figure 3.5B). In summary, individual p14-15 ANFs can already encode a similar range of stimulus levels as p20-21 fibers. The main difference was that p14-15 ANFs had higher thresholds compared to those of p20-21 fibers.
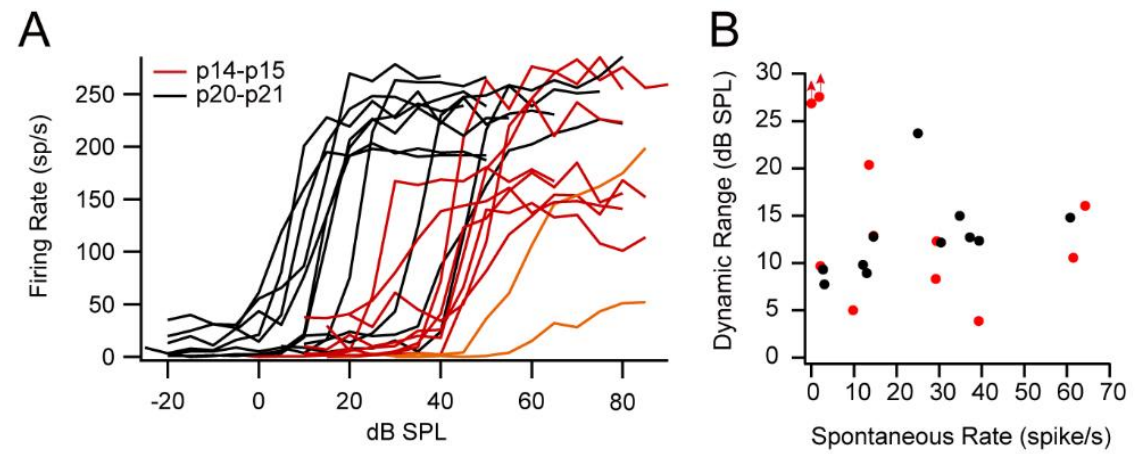

Figure 3.5: Changes of rate-level functions during early post natal development. (A) Example rate-level functions of p14-15 (red) and p20-21(black). (B) Similar dynamic ranges between p14-15 and p20-21 ANFs groups. Red arrows indicate two p14-15 fibers of low spontaneous rate $(<5 \mathrm{~Hz})$ showing non-saturating rate-level functions. Corresponding rate-level functions in (A) are shown in orange. 


\subsection{Synaptic ribbon and presynaptic structure in sound encoding: lessons from Bassoon mutants}

In the previous study, single fiber response properties during early postnatal development were characterized. We have discussed one potential hypothesis that presynaptic $\mathrm{Ca}^{2+}$ signaling and Cav1.3 channel organization causes heterogeneity of ANFs. Interestingly, the ANF heterogeneity seems to be correlated with the morphology and size of a special presynaptic structure in IHC: synaptic ribbon (Liberman 1980, Liberman, Wang et al. 2011). The presynaptic protein Bassoon has been shown to have a function in anchoring synaptic ribbon and organizing Cav1.3 channel clustering (Altrock, tom Dieck et al. 2003, Khimich, Nouvian et al. 2005, Frank, Rutherford et al. 2010). In this study, the advantage of molecular manipulation in mice was utilized to gain further insight into the role of presynaptic scaffold protein Bassoon and synaptic ribbon in sound encoding.

\subsubsection{Bsn $^{\text {gt }}$ IHCs ribbon anchorage phenotypes}

To study the morphology of IHC afferent synapses, confocal images of immunohistochemical labeling of postsynaptic AMPA receptors GluA2, CtBP2/ribeye (labeling synaptic ribbon/some nuceli) and presynaptic Cav1.3 $\mathrm{Ca}^{2+}$ channels of wholemounted organ of Corti (images acquired by Dr. Mark Rutherford) were analyzed. The number of afferent synapses per IHC was quantified by counting the number of postsynaptic marker GluA2-immunofluorescent spots in $\mathrm{Bsn}^{\mathrm{gt}}$ and $\mathrm{WT}_{\mathrm{gt}}$ images processed in parallel. In the 5-8 kHz tonotopic range, both $\mathrm{Bsn}^{\mathrm{gt}}$ and $\mathrm{WT}_{\mathrm{gt}}$ had an average of 11.4 afferent synapses per IHC (Figure 3.6A, n=2264 synapses in 199 IHCs vs. n=1682 synapses in 148 IHCs), indicating a normal connectivity. While in $\mathrm{WT}_{\mathrm{gt}} \mathrm{IHCs}$, nearly all GluA2 spots had a juxtaposed synaptic ribbon (97.2\%), most mutant synapses lacked juxtaposed ribbons $(56.4 \%, 4-11$ weeks with no clear age dependence), which were referred to as ribbonless synapses. Compared to $\mathrm{Bsn}^{\Delta \mathrm{Ex} 4 / 5}$, which showed an age dependent decrease of ribbon occupied synapses from $47 \%$ at p11 to $22 \%$ at p21 and $12 \%$ at p70 (Khimich, Nouvian et al. 2005, Jing, Rutherford et al. 2013), the loss of synaptic ribbon seems less severe in $\mathrm{Bsn}^{\mathrm{gt}}$. 
Previous electron microscopy (EM) data on a small set of Bsn ${ }^{\text {gt }}$ IHC synapses suggested a tendency of increased distance between synaptic ribbon and plasma membrane (Frank 2010). Here, a different approach was used to analyze the spatial organization of the synaptic structure. The 3D center of mass distances between CtBP2-, Cav1.3- and GluA2immunofluorescent spots in confocal image stacks were calculated for each triple cluster of juxtaposed CtBP2-, Cav1.3- and GluA2-immunofluorescence (see methods for details). The distance between CtBP2 and Cav1.3 spots was larger in Bsn ${ }^{\mathrm{gt}}$ IHCs compared to that at WT synapses (Figure $3.6 \mathrm{~B}, \mathrm{p}<0.001, \mathrm{n}=99$ synapses in $\mathrm{Bsn}^{\mathrm{gt}} \mathrm{vs.} \mathrm{n}=250$ synapses in $\mathrm{WT}_{\mathrm{gt}}$ ). The enlarged distance between CtBP2 spots to Cav1.3 and GluA2 spots in Bsn ${ }^{\mathrm{gt}}$ synapses confirmed the notion a looser ribbon anchorage to the presynaptic active zone. There was no significant difference for GluA2-Cav1.3 distances (Figure 3.6B, p=0.06, $\mathrm{n}=227$ synapses in $\mathrm{Bsn}^{\mathrm{gt}}$ vs. $\mathrm{n}=263$ synapses in $\mathrm{WT}_{\mathrm{gt}}$ ), arguing against a more general disruption of the synaptic ultrastructure in the absence of Bassoon.

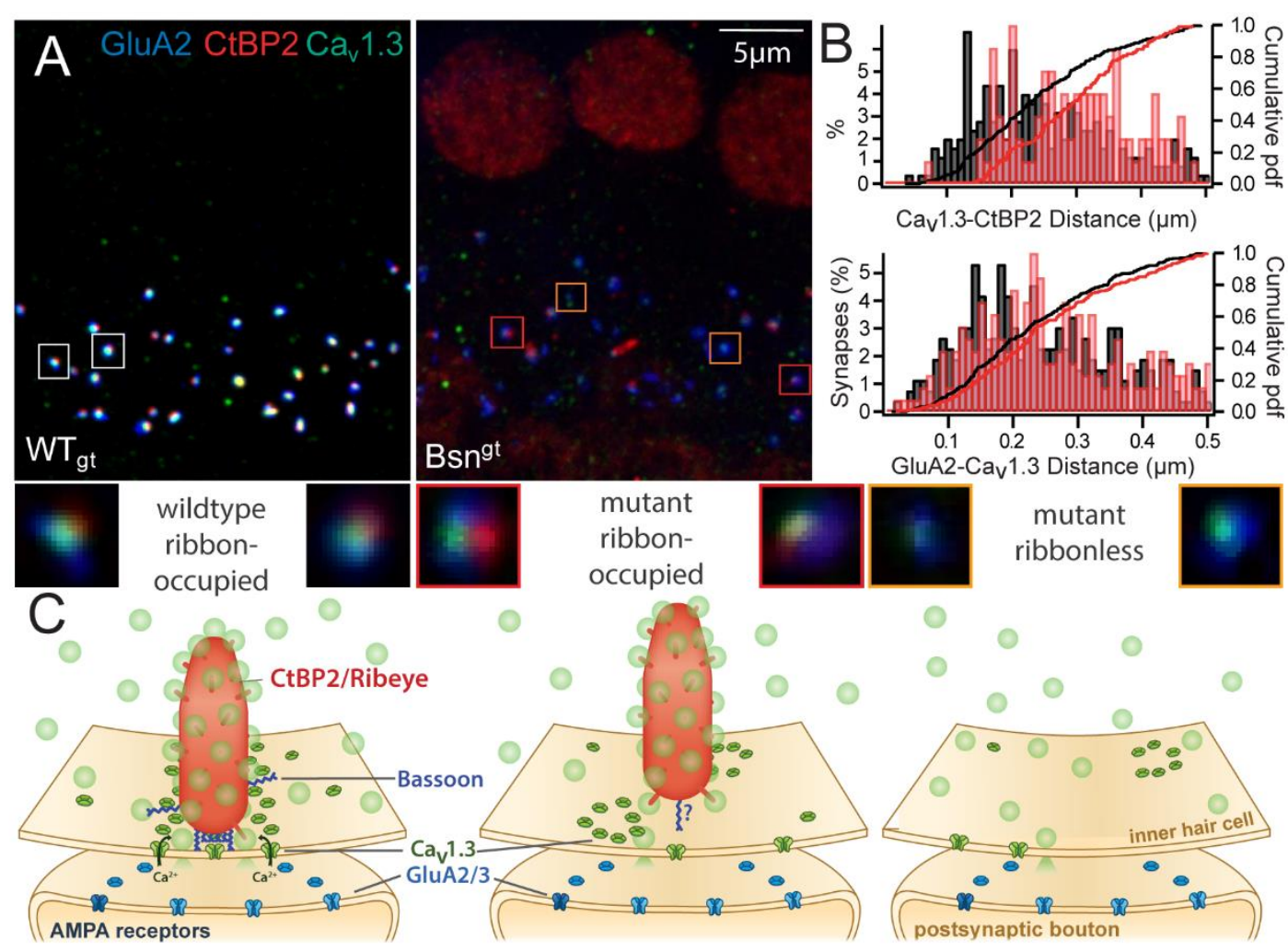

Figure 3.6: $3 \mathrm{D}$ confocal imaging analysis of synaptic organization in $\mathrm{Bsn}^{\mathrm{gt}}$ mutants. (A) IHCs stained with antibodies against GluA2 (blue, labeling postsynaptic glutamate receptors), CtBP2 (red, labeling synaptic ribbons and some nuclei) and Cav1.3 (green, labeling presynaptic $\mathrm{Ca}^{2+}$ channels). Note in both $\mathrm{Bsn}^{\text {gt }} \mathrm{IHCs}$, the number of GluA2 
spots was comparable to $\mathrm{WT}_{\mathrm{gt}}$, but the number of $\mathrm{CtBP} 2$ spots was reduced. (B) $3 \mathrm{D}$ center of mass distance distribution between pairs of $\mathrm{Ca}_{v} 1.3-\mathrm{CtBP} 2$ and GluA2-Cav1.3 positive clusters from one representative pair of images, showing enlarged ribbon distance to presynaptic active zone and comparable distance of pre- and post- synaptic markers. (C) Schematic illustration of active zone (AZ) organization in WT (left), mutant ribbon-occupied (middle) and mutant ribbonless synapses (right). Insect shows deconvolved high-magnification images of representative synapses. (Immunostaining images were obtained by Dr. Mark Rutherford, Cartoon illustration was drawn by Linda Hsu)

\subsubsection{Residual expression of bassoon in $\mathrm{BSN}^{\mathrm{gt}} \mathrm{IHCs}$}

It is not clear why the two mutants showed different synaptic ribbon occupancy phenotypes. $\mathrm{Bsn}^{\Delta \mathrm{Ex} 4 / 5}$ mutant mice were generated by knock out the exon 4 and 5 of bassoon gene, resulting an expression of mRNA fragment of bassoon. The mRNA fragment encodes for a $180 \mathrm{kD}$ protein fragment. However, the fragment did not appear to be synaptically localized in immunohistochemistry studies of the brain (Altrock, tom Dieck et al. 2003) and hair cells (Frank, Rutherford et al. 2010). Morphology and physiology in heterozygous mice did not support a dominant negative function of the fragment. In contrast, the $\mathrm{Bsn}^{\mathrm{gt}}$ mice were generated by inserting a termination sequence between exons 1 and 2 of bassoon gene to block the transcription. No residual bassoon expression was found in Western blots and immunohistochemistry of brain tissue (Hallermann, Fejtova et al. 2010). However, residual expression of a small amount of whole-length mRNA could not be excluded. We therefore performed real-time PCR and immunohistochemistry studies.

The residual expression of bassoon was first investigated at mRNA level. Real-time PCR of organ of Corti from Bsn ${ }^{\text {gt }}$ mice was carried out to test possible bassoon mRNA. A residual expression of bassoon mRNA in $\mathrm{Bsn}^{\mathrm{gt}}$ organs of Corti amounted to $3 \%$ of wildtype levels was found (6 organ of Corti each, data not shown).

The expression of bassoon at protein level was investigated by immunolabeling Bassoon, synaptic ribbons (CtBP2), and Cav1.3 channels (Performed by Dr. Mark Rutherford). Bassoon-immunolabeling was detected at virtually all afferent wildtype IHC synapses that were colabeled by CtBP2 and Cav1.3 (Figure 3.7A). However, in Bsn ${ }^{\text {gt }}$ IHCs, bassoon immunoreactivity was bared detectable in the triple staining preparations (Figure 
3.7B). Here, a possible bassoon immunofluorescence was found at one synapse out of hundreds of synapses in 72 IHCs (Figure 3.7B, white frame). In another preparation of double-labeled for $\mathrm{Ca}_{\mathrm{v}} 1.3$ and bassoon, weak bassoon immunoreactivity was found overlapping with $\mathrm{Ca}_{v} 1.3$ clusters at $<5 \%$ of synapses (Frank, Rutherford et al. 2010).

Taken together, a small amount of leaky expression was detected by Real-time PCR experiments, which was barely detectable at protein level. A few copies of molecules may support the synaptic anchorage of the ribbon in $\mathrm{Bsn}^{\mathrm{gt}}$ IHCs, but are not sufficient to support the normal tight spatial coupling of synaptic ribbon to the presynaptic membrane.
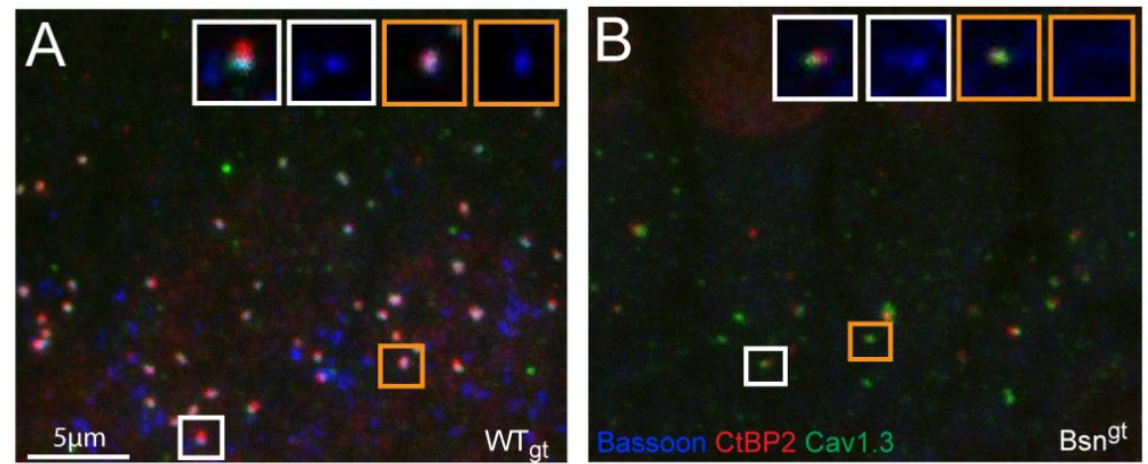

Figure 3.7: Leaky expression of bassoon in a subset of Bsn ${ }^{\text {gt }}$ IHCs. IHCs were stained with antibodies against Bassoon (blue), synaptic ribbons (CtBP2, red) and Cav1.3 channels (green). (A) At synapses of $\mathrm{WT}_{\mathrm{gt}}$, Bassoon clustered at juxtaposition to CtBP2 and Cav1.3 colabeled spots. Additional bassoon-positive, but CtBP2- and Cav1.3negative spots represent efferent synapses contacting postsynaptic structures. Insets show two representative examples at all three channels and bassoon channel only. (B) At synapses of $\mathrm{Bsn}^{\text {gt }}$, Bassoon punctas were barely detectable by visual inspection. Insects show a weak bassoon-positive synapse (white frame) and a synapse with bassoon immunofluorescence not exceeding background level (golden frame). (Images were obtained by Dr. Mark Rutherford)

\subsubsection{Ribbon-dependent effects on synaptic morphology and physiology in Bsn ${ }^{\text {gt }}$ IHCs}

The observation of a higher fraction of ribbon-occupied synapses triggers the question, whether it is possible to scrutinize ribbon-dependent effects on the presynaptic active zone using the Bsn ${ }^{\mathrm{gt}}$ mutant? If those looser anchored ribbons were functional different properties of the $47.3 \%$ ribbon-occupied and $52.7 \%$ ribbonless synapses, it should be 
observed in the $47.3 \%$ ribbon-occupied synapses. Previous results suggested a possible role of synaptic ribbons in organizing Cav1.3 channels (Frank, Rutherford et al. 2010). Triple immunolabeling of CtBP2, Cav1.3 and GluA2 of organs of Corti (staining performed by/in collaboration with Dr. Mark Rutherford; Figure 3.6A) enable us to study ribbon-dependent effects on synaptic organizations in Bsn ${ }^{\mathrm{gt}}$ IHCs. Immunofluorescence intensities of CtBP2, Cav1.3 and GluA2 spots were quantitatively analyzed. Using GluA2 as a synaptic marker, the synaptic Cav1.3 immunofluorescence intensity was found to be reduced compared to $\mathrm{WT}_{\mathrm{gt}}$ IHCs. Ribbon-occupied synapses had significantly stronger Cav1.3 immunofluorescence than ribbonless synapses in Bsn ${ }^{\mathrm{gt}} \mathrm{IHCs}$ (Figure 3.8A,B). In contrast, the mean intensities of GluA2 spots were not significantly different between genotypes and the distributions were nearly indistinguishable regardless of the presence or absence of a ribbon (Figure 3.8C). The GluA2 organization was also assessed in $\mathrm{Bsn}^{\triangle \mathrm{Ex} 4 / 5}$ IHCs using STED microscopy by Drs. Mark Rutherford and Benjamin Harke. In mutant synapses, the ring-like organization of GluA2 clusters was retained, albeit with slightly smaller diameter in the absence of a ribbon (Figure 3.8D).
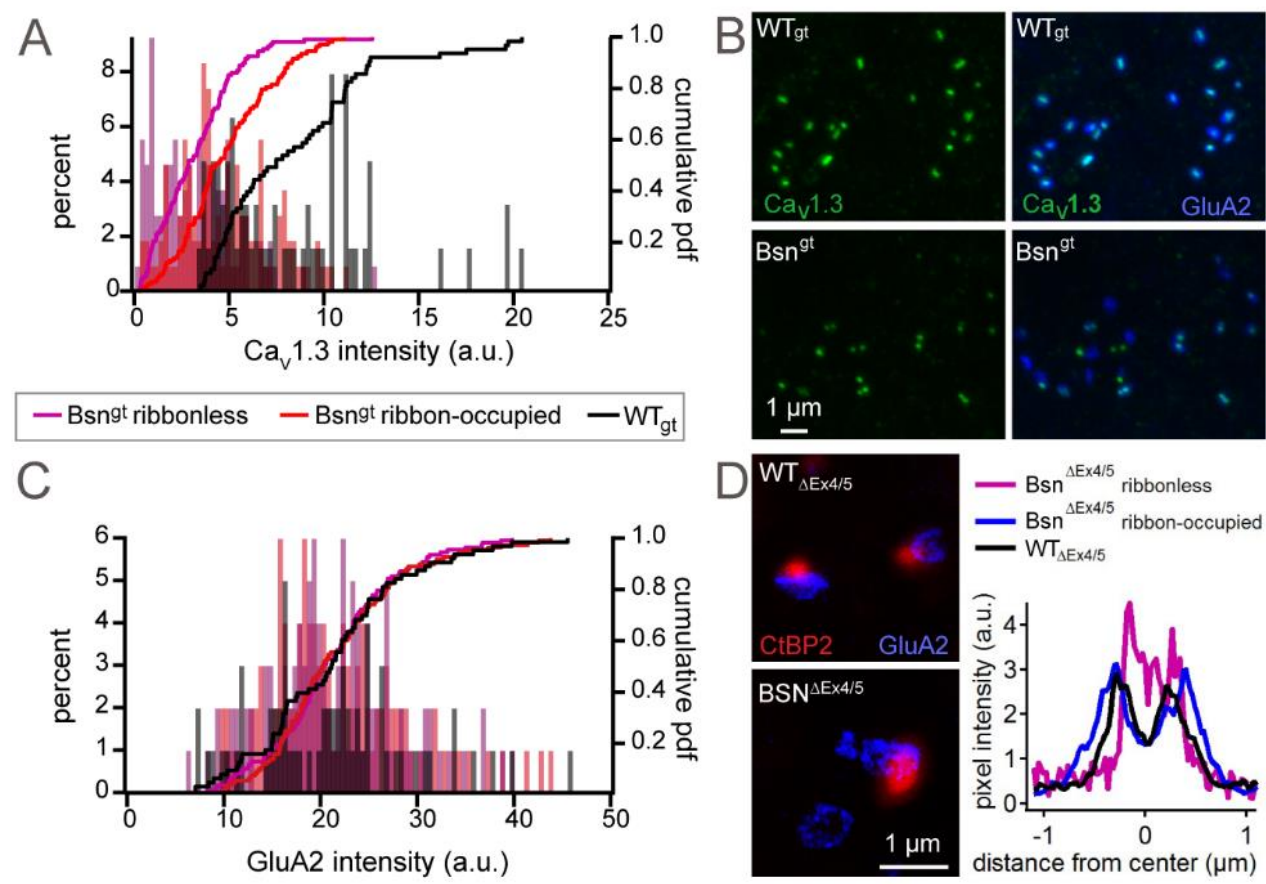

Figure 3.8: Ribbon dependent morphological differences in Bsn ${ }^{\text {gt }}$ IHCs. (A, C) Quantification of the representative pair of triple labelings shown in Figure 3.6. Cav1.3 immunofluorescence intensity in $\mathrm{Bsn}^{\text {gt }} \mathrm{IHCs}$ (violet, $\mathrm{n}=108 \mathrm{Bsn}^{\text {gt }}$ ribbonless synapses; red, $\mathrm{n}=108$ ribbon-occupied synapses) had reduced intensity compared to $\mathrm{WT}_{\mathrm{gt}}$ (black, $\mathrm{n}=105$ synapses). Ribbon-occupied $\mathrm{Bsn}^{\mathrm{gt}}$ synapses had more Cav 1.3 immunofluorescence than ribbonless synapses but less than $\mathrm{WT}_{\mathrm{gt}}$. However, the 
distributions of GluA2 cluster immunofluorescence intensities of $\mathrm{Bsn}^{\mathrm{gt}}$ and $\mathrm{WT}_{\mathrm{gt}}$ synapses were not different (C). (B) Representative pair of double-staining of GluA2 (blue) and Cav1.3 (green), showing reduced Cav1.3 but not GluA2 receptor immunofluorescence intensities in the Bsn ${ }^{\text {gt }}$ IHCs. (D) STED microscopy of Bsn ${ }^{\triangle E \times 4 / 5}$ IHCs stained for GluA2 receptors (blue) and synaptic ribbons (CtBP2, red). Both $\mathrm{WT}_{\mathrm{gt}}$ and $\mathrm{Bsn}^{\Delta \mathrm{Ex} 4 / 5}$ synapses retained the ring-like morphology of GluA2 clusters. However, in the absence of a presynaptic ribbon, the ring diameters appeared slightly smaller (Data from Drs. Mark Rutherford, Benjamin Harke).

To correlate the morphological differences to IHC physiology, in vitro cell physiology experiments were carried out by Dr. Thomas Frank. Both mutants had reduced whole cell $\mathrm{Ca}^{2+}$ currents (Figure 3.9A), consistent with the reduced Cav1.3 immunofluorescence as previously described (Figure 3.8A, B). The maximum $\mathrm{Ca}^{2+}$ current in $\mathrm{Bsn}^{\mathrm{gt}}$ was intermediate between $\mathrm{Bsn}^{\Delta \mathrm{Ex} 4 / 5}$ and WT IHCs. This is consistent with a milder physiological phenotype and function of synaptic ribbons in stabilizing presynaptic Cav1.3 clusters (Frank, Rutherford et al. 2010). The voltage-dependence of $\mathrm{Ca}^{2+}$ channels was not different among IHCs of the different genotypes. To probe the exocytosis of IHCs, capacitance measurements were carried out by depolarizing the IHC to the potential of maximum $\mathrm{Ca}^{2+}$ current for $20 \mathrm{~ms}$ (Figure 3.9B) and $100 \mathrm{~ms}$. The capacitance change during the first $20 \mathrm{~ms}$ can be interpreted as the size of readily releasable pool (Moser and Beutner 2000), while the capacitance changes between $100 \mathrm{~ms}$ and $20 \mathrm{~ms}$ reflected the sustained component of exocytosis. There was no difference between the RRP size between $\mathrm{Bsn}^{\mathrm{gt}}$ and $\mathrm{Bsn}^{\Delta \mathrm{Ex} 4 / 5}$. However, Bsn ${ }^{\mathrm{gt}} \mathrm{IHCs}$ had a significantly larger sustained component of exocytosis compared to that of $\mathrm{Bsn}^{\Delta \mathrm{Ex} 4 / 5} \mathrm{IHCs}$ (Figure 3.9C), suggesting that vesicle replenishment is better preserved. Intracellular application of the slowbinding $\mathrm{Ca}^{2+}$ chelator 5mM EGTA in whole-cell recordings did not cause a stronger relative suppression of exocytosis in either Bsn mutant, suggesting an unaltered spatial coupling between synaptic $\mathrm{Ca}^{2+}$ channels and release-ready vesicles (Figure 3.9C, D). The recovery of RRP upon depletion was probed using pair-pulse protocol, during which the first $20 \mathrm{~ms}$ or $100 \mathrm{~ms}$ depolarization depleted the pool. After various different intervals, another depolarization of the same duration was used to test the recovery of the RRP after depletion. The recovery rate in $\mathrm{Bsn}^{\mathrm{gt}}$ was again intermediate between $\mathrm{Bsn}^{\Delta \mathrm{Ex} 4 / 5}$ and $\mathrm{WT}$ IHCs. In summary, the cell physiology confirmed that Bsn ${ }^{\text {gt }}$ IHCs with their larger fraction of ribbon-occupied synapses show better preserved presynaptic function than $\mathrm{Bsn}^{\Delta \mathrm{Ex} 4 / 5} \mathrm{IHCs}$, which mostly lack synaptic ribbons. The residual looser anchored 
synaptic ribbons in $\mathrm{Bsn}^{\mathrm{gt}} \mathrm{IHCs}$ support better maximum whole cell $\mathrm{Ca}^{2+}$ current, sustained exocytosis and replenishment of RRP, but do not maintain a normal size of the RRP.
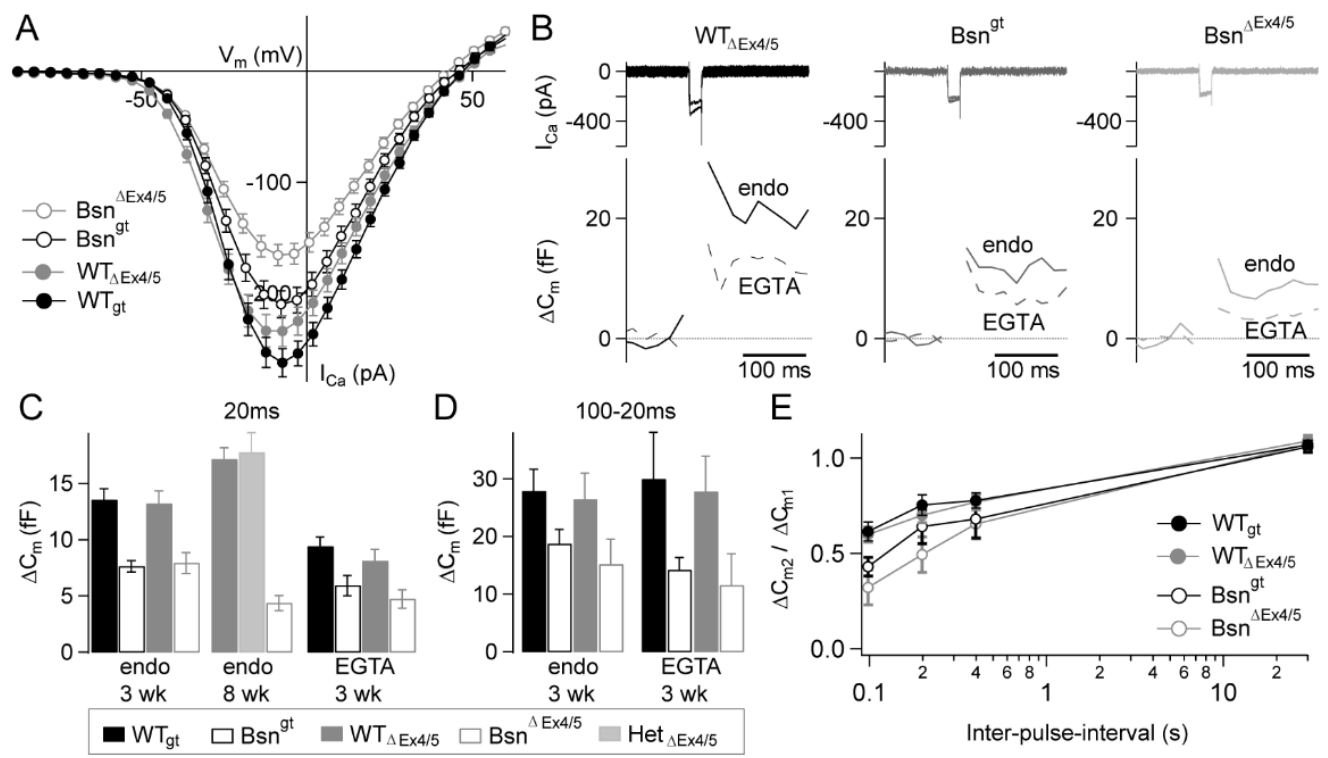

$\mathrm{E}$

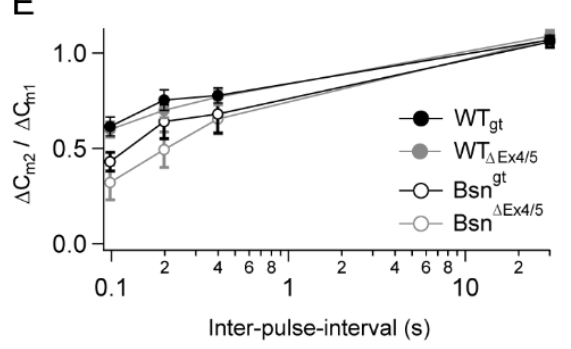

Figure 3.9: Effect of Bassoon disruption on synaptic functions. (A) $\mathrm{Ca}^{2+}$ currentvoltage relationships. $\mathrm{Bsn}^{\Delta \mathrm{Ex} 4 / 5} \mathrm{IHCs}$ had reduced peak $\mathrm{Ca}^{2+}$ current compared to their respective control $\left(80 \%, \mathrm{p}<0.01\right.$ for $\mathrm{Bsn}^{\mathrm{gt}} ; 71 \%, \mathrm{p}<0.001$ for $\mathrm{Bsn}^{\Delta \mathrm{Ex} 4 / 5} ; \mathrm{n}=24$ for $\mathrm{WT}_{\mathrm{gt}}$, $\mathrm{n}=26$ for $\mathrm{Bsn}^{\mathrm{gt}}, \mathrm{n}=43$ for $\mathrm{WT}_{\Delta \mathrm{Ex} 4 / 5}, \mathrm{n}=46$ for $\mathrm{Bsn}^{\Delta \mathrm{Ex} 4 / 5}$ ). (B) Representative traces of perforated patch clamp experiments on IHCs, illustrating a comparable reduction in capacitance jumps following $20 \mathrm{~ms}$ depolarization to the membrane potential eliciting the maximum $\mathrm{Ca}^{2+}$ current in Bsn mutants compared to wildtype IHCs (mean reduction of $40 \%$ in $\mathrm{Bsn}^{\Delta \mathrm{Ex} 4 / 5}$ and $44 \%$ in $\mathrm{Bsn}^{\mathrm{gt}}$ ). Replacing endogenous intracellular buffers by 5 $\mathrm{mM}[\mathrm{EGTA}]_{\mathrm{i}}$ led to a comparable reduction in exocytosis in all genotypes. (C-D) Average $\Delta \mathrm{C}_{\mathrm{m}}$ for indicated pulse durations. Only the sustained component $\left(\Delta \mathrm{C}_{\mathrm{m}} 100-\right.$ $\left.\Delta \mathrm{C}_{\mathrm{m}} 20\right)$ was significantly more affected in $\mathrm{Bsn}^{\Delta \mathrm{Ex} 4 / 5}$ than in $\mathrm{Bsn}^{\mathrm{gt}} \mathrm{IHC}$. Comparable relative reduction of exocytosis in the presence of $5 \mathrm{mM}$ [EGTA] $]_{\mathrm{i}}$ suggests comparable coupling of vesicle fusion to presynaptic $\mathrm{Ca}^{2+}$ influx in both mutants. ( $\mathrm{n}=24$ for $\mathrm{WT}_{\mathrm{gt}}$, $\mathrm{n}=25$ for $\mathrm{Bsn}^{\mathrm{gt}}, \mathrm{n}=38$ for $\mathrm{WT}_{\Delta \mathrm{Ex} 4 / 5}, \mathrm{n}=36$ for $\mathrm{Bsn}^{\Delta \mathrm{Ex} 4 / 5}$ ). (E) There was a tendency for more pronounced vesicle pool depletion in $\mathrm{Bsn}^{\Delta \mathrm{E} \times 4 / 5}$ compared to $\mathrm{Bsn}^{\mathrm{gt}}$ in paired pulse experiments ( $\mathrm{n} \geq 20$ for $\mathrm{WT}_{\mathrm{gt}}, \mathrm{n} \geq 13$ for $\mathrm{Bsn}^{\mathrm{gt}}, \mathrm{n} \geq 23$ for $\mathrm{WT}_{\Delta \mathrm{Ex} 4 / 5}, \mathrm{n} \geq 20$ for $\mathrm{Bsn}^{\Delta \mathrm{Ex} 4 / 5}$; number provides minimum available for all intervals). (Data from Dr. Thomas Frank)

\subsubsection{Sound encoding phenotypes in $\mathrm{Bsn}^{\mathrm{gt}}$ and $\mathrm{Bsn}^{\Delta \mathrm{Ex} 4 / 5}$ ANFs}

Having observed ribbon-dependent effects on Cav1.3 channel clusters and IHC cell physiology, it was interesting to test for ribbon-dependent effects on sound encoding in ANFs. The in vitro physiology data were obtained from whole IHCs, which summed the 
responses of 10-20 active zones and potential $\mathrm{Ca}^{2+}$ influx and exocytosis at the extrasynaptic membrane. This method is not able to address the ribbon dependent effect on sound encoding at the individual synapse level. Individual synapses can be studied by recording from postsynaptic boutons, where the afferent fibers make contact with IHCs (Glowatzki and Fuchs 2002, Grant, Yi et al. 2011). However, it is difficult to apply this technically challenging experiment to adult mice. Dr. Hideki Takago successfully recorded from $\mathrm{Bsn}^{\Delta \mathrm{Ex} 4 / 5}$ postsynaptic afferent boutons of type I SGNs at postnatal days 911. At that age, the fraction of ribbon-occupied synapses in IHCs was $\sim 47 \%$. Results were in agreement with the impaired exocytosis and vesicle replenishment in Bsn mutants. The EPSCs frequency were reduced at rest $\left(0.11 \pm 0.03 \mathrm{~Hz}\right.$ in $\mathrm{Bsn}^{\Delta \mathrm{Ex} 4 / 5}, \mathrm{n}=7 ; 0.79 \pm 0.26 \mathrm{~Hz}$ in $\left.\mathrm{WT}_{\Delta \mathrm{Ex} 4 / 5}, \mathrm{n}=5, \mathrm{p}<0.05\right)$ and during application of $40 \mathrm{mM} \mathrm{K}^{+}\left(0.59 \pm 0.34 \mathrm{~Hz}_{\text {in }} \mathrm{Bsn}^{\Delta \mathrm{Ex} 4 / 5}\right.$, $\mathrm{n}=5 ; 10.9 \pm 3.70 \mathrm{~Hz}$ in $\left.\mathrm{WT}_{\Delta \mathrm{Ex} 4 / 5}, \mathrm{n}=5, \mathrm{p}<0.05\right)$. Every $\mathrm{WT}_{\Delta \mathrm{Ex} 4 / 5}$ and $\mathrm{Bsn}^{\Delta \mathrm{Ex} 4 / 5}$ bouton had EPSCs with smooth rise and fall (monophasic) and more complex waveforms (multiphasic). The amplitude of both monophasic and multiphasic EPSCs were on average two fold smaller in $\mathrm{Bsn}^{\Delta \mathrm{Ex} 4 / 5}$ afferent, but showed unaltered kinetics. No segregation of postsynaptic responses into different groups was observed, but this analysis was hindered by the paucity of events.

\subsubsection{Spontaneous discharge activity}

An alternative method for single synapse analysis is to record directly from auditory nerve fibers. Here, single-unit recordings from ANFs were used to address the question above. Responses of ANFs from Bsn ${ }^{\mathrm{gt}}, \mathrm{Bsn}^{\Delta \mathrm{Ex} 4 / 5}, \mathrm{WT}_{\mathrm{gt}}, \mathrm{WT}_{\Delta \mathrm{Ex} 4 / 5}$ and $\mathrm{HZ}_{\Delta \mathrm{Ex} 4 / 5}$ (heterozygous) were compared under identical experimental conditions. Each ANF was first characterized by assessing its spontaneous discharge activity in the absence of sound stimuli. As previously reported (Buran, Strenzke et al. 2010), $\mathrm{Bsn}^{\Delta \mathrm{Ex} 4 / 5}$ ANFs lacked fibers with high spontaneous rate (Figure 3.9B2). Bsn ${ }^{\text {gt }}$ ANFs showed a similar phenotype (Figure 3.10B1). In both mutants, the spontaneous firing rate never exceeded 50 spike/s, while some WT fibers spontaneously fired at a rate over 100 spike/s.

The time pattern of spontaneous discharge activity is highly irregular. It can be described as a "Poisson process", where the interval between successive spikes is drawn randomly from an exponential function with a dead time (Kiang 1965). Representative inter-spike 
interval histogram with similar spontaneous rate from four genotypes were shown in Figure 3.10A. The irregular, Poissonian spike pattern was retained in both mutants. To quantify the regularity of spontaneous firing, the coefficient of variation of inter-spike interval was computed for each fiber with non-zero spontaneous firing rate. There was no difference between the CVs of $\mathrm{Bsn}^{\mathrm{gt}}$ and $\mathrm{Bsn}^{\Delta \mathrm{Ex} 4 / 5}$ ANFs $\left(0.99 \pm 0.03, \mathrm{n}=43\right.$ for Bsn ${ }^{\text {gt; }} ; 0.98$ $\pm 0.02, \mathrm{n}=31$ for $\left.\mathrm{Bsn}^{\Delta \mathrm{Ex} 4 / 5}\right)$, as well as between $\mathrm{WT}_{\mathrm{gt}}$ and $\mathrm{WT}_{\Delta \mathrm{Ex} 4 / 5} \mathrm{ANFs}(0.89 \pm 0.03$, $\mathrm{n}=29$ for $\mathrm{WT}_{\mathrm{gt}} ; 0.94 \pm 0.04, \mathrm{n}=23$ for $\left.\mathrm{WT}_{\Delta \mathrm{Ex} 4 / 5}\right)$.
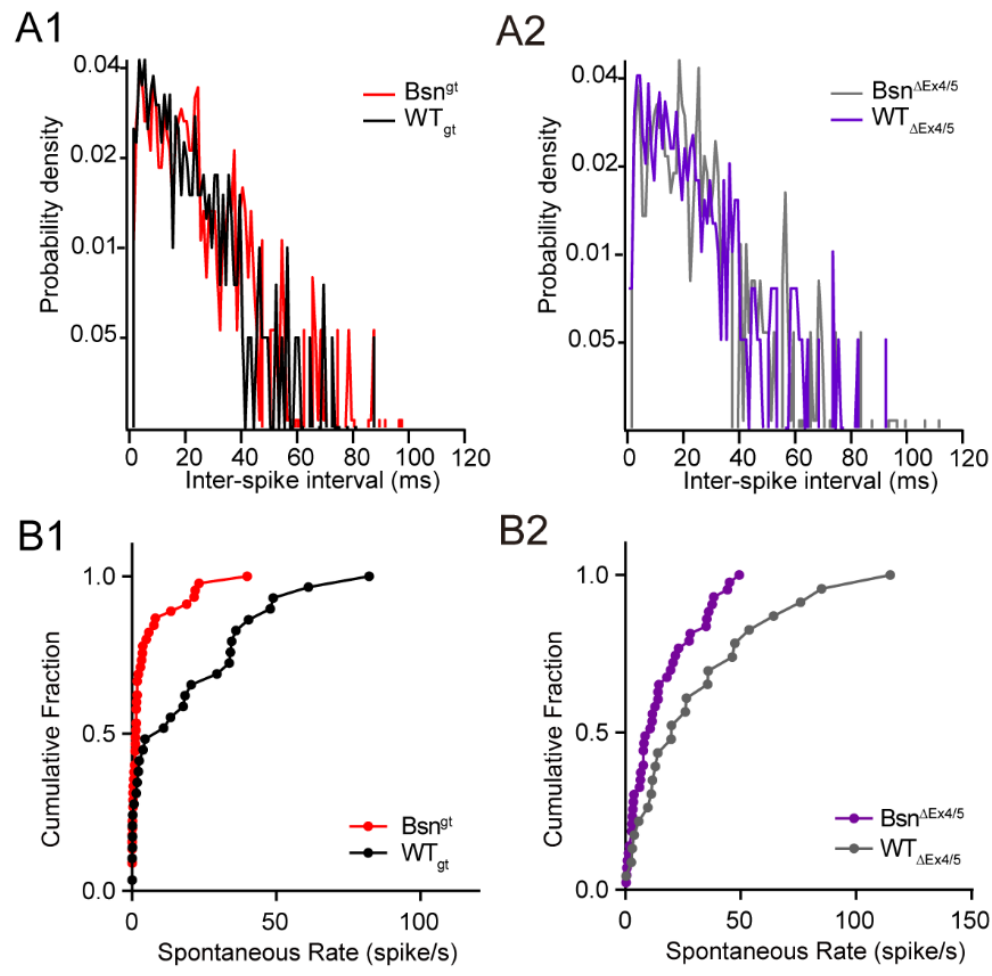

Figure 3.10: Time pattern of spontaneous activities and lack of high spontaneous rate fibers in Bsn mutants. (A1-A2) Representative inter-spike interval histogram of fibers with similar spontaneous rates $\left(\mathrm{WT}_{\mathrm{gt}}: 40.4\right.$ spikes/s, Bsn ${ }^{\mathrm{gt}}: 39.9$ spikes/s, $\mathrm{WT}_{\Delta \mathrm{Ex} 4 / 5}: 35.6$ spikes/s, Bsn ${ }^{\Delta \mathrm{Ex} 4 / 5}: 38.3$ spikes/s). (B1-B2) Cumulative histogram of spontaneous discharge rate across fibers indicated a lack of high spontaneous rate fibers in both mutants.

\subsubsection{Tuning curves and threshold}

Next, tuning curves of ANFs from Bsn wild-types and mutants were characterized. The hearing frequency range differed for the two mouse strains, presumably due to differences 
in the genetic background. Most ANFs from $\mathrm{Bsn}^{\mathrm{gt}}$ and $\mathrm{WT}_{\mathrm{gt}}$ mice had CFs ranging between 16 and $50 \mathrm{kHz}$, while most $\mathrm{ANFs}$ from $\mathrm{Bsn}^{\Delta \mathrm{Ex} 4 / 5}$ and $\mathrm{WT}_{\Delta \mathrm{Ex} 4 / 5}$ mice had CFs ranging between 8 and $30 \mathrm{kHz}$. Bsn ${ }^{\mathrm{gt}}$ and $\mathrm{Bsn}^{\Delta \mathrm{Ex} 4 / 5}$ had a similar range of CFs and normal thresholds when comparing to their respective littermate controls (Figure 3.11A1, B1).

The sharp frequency selectivity requires normal mechanical amplification function of OHCs. Distortion product otoacoustic emissions (DPOAE) showed no significant differences (Khimich, Nouvian et al. 2005, Buran, Strenzke et al. 2010), arguing against an amplification problem in Bsn mutants. Consistent with this observation, the sharpness of tuning for individual $\mathrm{Bsn}^{\mathrm{gt}}$ and $\mathrm{Bsn}^{\Delta \mathrm{Ex} 4 / 5}$ fibers, quantified as Q10 $\mathrm{dB}$, was comparable to that of their respective controls (Figure 3.11A2, B2).
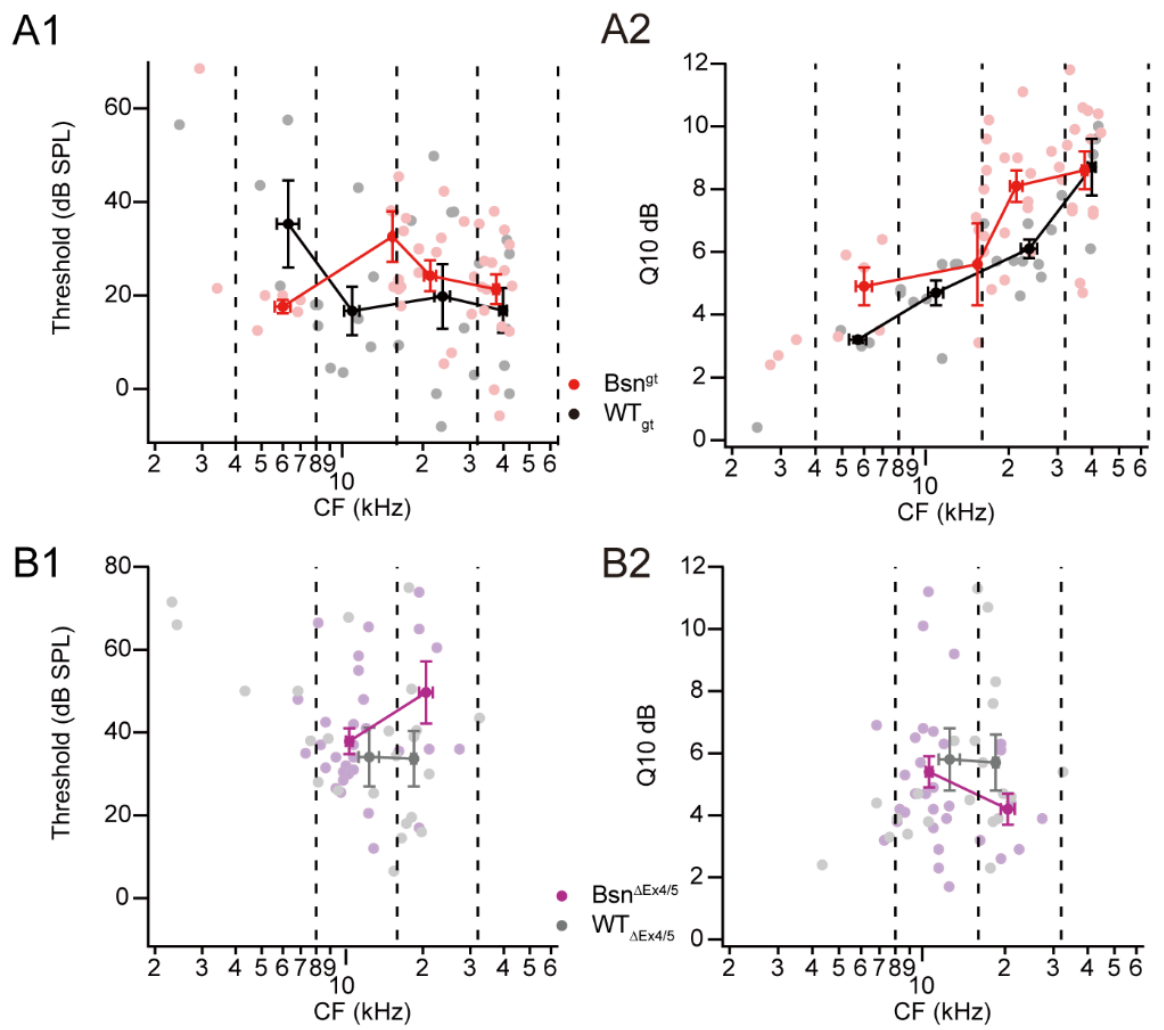

Figure 3.11: Normal auditory nerve fiber thresholds and sharpness of tuning of tuning curves. (A1, B1) Thresholds at CF for $45 \mathrm{Bsn}^{\mathrm{gt}}, 29 \mathrm{WT}_{\mathrm{gt}}(\mathrm{A} 1), 31 \mathrm{Bsn}^{\Delta \mathrm{Ex} 4 / 5}$ and 23 $\mathrm{WT}_{\Delta \mathrm{Ex} 4 / 5}$ (B1) fibers. The filled round circles and lines show the mean threshold as a function of CF. Data were binned according to CF into four groups (dotted vertical line). Mean thresholds for both mutants in each bin were not significantly different. Note more fibers of high CFs $(>32 \mathrm{kHz})$ presented in the sample in genetrap strain (A1) compared to the partial deletion strain (B1). (A2, B2) Sharpness of tuning as a function of $\mathrm{CF}$ for the same population of fibers in $\mathrm{A} 1$ and $\mathrm{B} 1$. Larger $\mathrm{Q} 10 \mathrm{~dB}$ indicates sharper 
tuning curves. Bsn ${ }^{\text {gt }}$ ANFs tuned between $16 \mathrm{kHz}$ and $32 \mathrm{kHz}$ on average had sharper tuning curves $(\mathrm{p}<0.01)$.

\subsubsection{Responses to tone burst stimulation}

If the remaining ribbons in $\mathrm{Bsn}^{\mathrm{gt}} \mathrm{IHCs}$ can support normal synaptic function in sound encoding, we might be able to separate the ANF responses into two groups, one from ribbon-occupied synapses, and the other from ribbonless synapses.

ANF responses in Bsn mutants to $50 \mathrm{~ms}$ tone bursts stimulation presented at $30 \mathrm{~dB}$ above threshold at $\mathrm{CF}$ showed typical high instantaneous firing rate at sound onset followed by adaptation (Figure 3.12A, B). The response latency was quantified as median first spike latency. In both Bsn mutants, the average median first spike latencies were longer (Figure $3.12 \mathrm{C}$ ), possibly due to failure of firing an action potential at stimulus onset. Adaptation was likely dominated by the depletion of the presynaptic RRP of vesicles (Moser and Beutner 2000, Goutman and Glowatzki 2007, Buran, Strenzke et al. 2010). Bsn ${ }^{\mathrm{gt}}$ and $\mathrm{Bsn}^{\Delta \mathrm{Ex} 4 / 5}$ showed similar rapid $\left(0.34 \pm 0.07 \mathrm{~ms}\right.$ for $\mathrm{Bsn}^{\text {gt. }} 0.36 \pm 0.08 \mathrm{~ms}$ for $\left.\mathrm{Bsn}^{\Delta \mathrm{Ex} 4 / 5}\right)$ and slow $\left(16.0 \pm 1.6 \mathrm{~ms}\right.$ for $\mathrm{Bsn}^{\mathrm{gt}} ; 13.1 \pm 1.9 \mathrm{~ms}$ for $\left.\mathrm{Bsn}^{\Delta \mathrm{Ex} 4 / 5}\right)$ adaptation time constants, which were not different from those of their respective controls (rapid component: 0.64 $\pm 0.19 \mathrm{~ms}$ for $\mathrm{WT}_{\mathrm{gt}} ; 0.37 \pm 0.07 \mathrm{~ms}$ for $\mathrm{WT}_{\Delta \mathrm{Ex} 4 / 5}$; slow component: $15.6 \pm 2.5 \mathrm{~ms}$ for $\mathrm{Bsn}^{\mathrm{gt}} ; 14.7 \pm 1.5 \mathrm{~ms}$ for $\mathrm{Bsn}^{\Delta \mathrm{Ex} 4 / 5}$ ). Both mutants also showed similar reduction in onset and adapted firing rate (Figure 3.12D). No obvious segregation into groups of $\mathrm{Bsn}^{\mathrm{gt}} \mathrm{ANFs}$ was observed, which would possibly indicate a difference between ribbon-occupied and ribbonless synapses. 

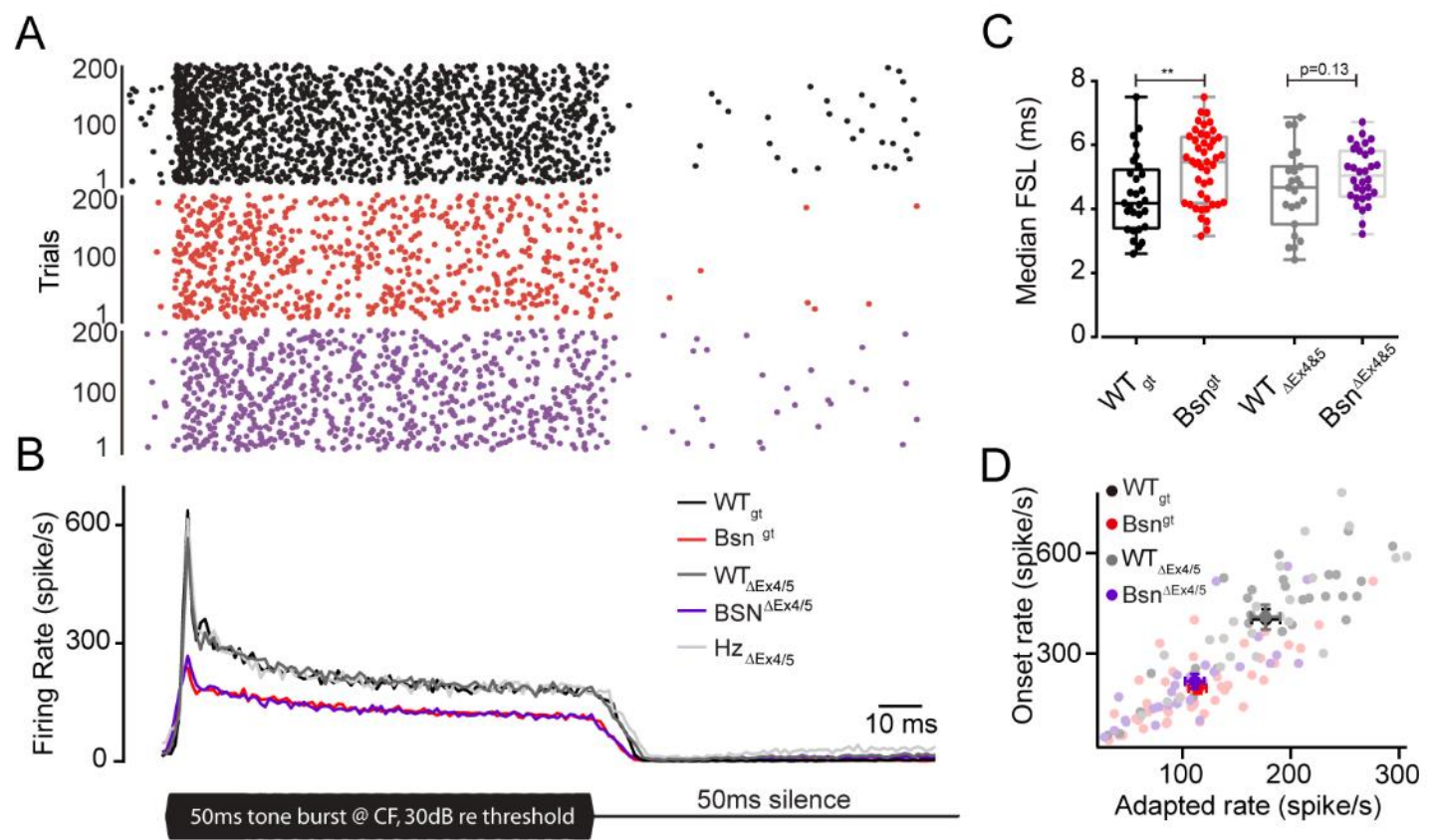

Figure 3.12: The sound encoding of tone bursts were affected to a similar extent for both Bsn mutants. (A) Representative ANF evoke response to a tone burst presented 30 $\mathrm{dB}$ above threshold at the CF. (B) Average post-stimulus-time histogram of Bsn mutants. Evoked firing rate were reduced to a similar extent in both mutants but with unaltered adaptation. Fibers were aligned to the bin of median first spike latency before averaging. (C) Measuring response latency by median first spike latency. Bsn ${ }^{\mathrm{gt}}$ fibers had significantly longer median first spike latency compared to $\mathrm{WT}_{\mathrm{gt}}(5.3 \pm 0.2 \mathrm{~ms}, \mathrm{n}=45$ for $\mathrm{Bsn}^{\mathrm{gt}}$ vs. $4.4 \pm 02 \mathrm{~ms}, \mathrm{n}=29$ for $\left.\mathrm{WT}_{\mathrm{gt}}, \mathrm{p}<0.01\right)$. There was also a tendency of longer median first spike latency for $\mathrm{Bsn}^{\Delta \mathrm{Ex} 4 / 5}$ fibers $\left(5.1 \pm 0.2 \mathrm{~ms} n=32\right.$ for $\mathrm{Bsn}^{\Delta \mathrm{Ex} 4 / 5} \mathrm{vs.} 4.6 \pm 0.3$ $\mathrm{ms}, \mathrm{n}=23$ for $\mathrm{WT}_{\Delta \mathrm{Ex} 4 / 5}, \mathrm{p}=0.13$ ). (D) Quantifying the firing rate for each individual unit indicated similar reduction in the onset $\left(401.7 \pm 30.2\right.$ spikes/s for $\mathrm{WT}_{\mathrm{gt}} \mathrm{vs} .408 .7 \pm 36.6$ spikes/s for $\mathrm{WT}_{\Delta \mathrm{Ex} 4 / 5} ; 195.9 \pm 15.6$ for $\mathrm{Bsn}^{\mathrm{gt}}$ vs. $216.4 \pm 22.0$ for $\left.\mathrm{Bsn}^{\Delta \mathrm{Ex} 4 / 5}\right)$ and adapted firing rate $\left(177.0 \pm 13.2\right.$ spikes/s for $\mathrm{WT}_{\mathrm{gt}}$ vs. $176.6 \pm 13.7$ spikes $/ \mathrm{s}$ for $\mathrm{WT}_{\Delta \mathrm{Ex} 4 / 5}$; 113.9 \pm 8.1 spikes/s for $\mathrm{Bsn}^{\text {gt }}$ vs. $111.3 \pm 8.7$ spikes/s for $\mathrm{Bsn}^{\Delta \mathrm{Ex} 4 / 5}$ ).

The recovery from adaptation likely reflects the replenishment of the RRP. Previously, paired-pulse experiments in whole cell physiology were used to probe the process (Figure 3.9E). Here, an in vivo forward-masking paradigm was applied. Similar to the pairedpulse protocol, a $100 \mathrm{~ms}$ tone burst was first presented, followed by another $15 \mathrm{~ms}$ probe tone separated by varying silent intervals. The probe evoked firing rate was normalized to adapted rate and masker evoked onset rate in same duration to assess the time course of recovery. According to the postulated role of Bassoon/synaptic ribbon in vesicle replenishment that was also indicated in previous capacitance recording, a slower 
recovery was expected, which might be more pronounced in Bsn ${ }^{\mathrm{gt}}$ ANFs. However, similar time courses of recovery in $\mathrm{Bsn}^{\mathrm{gt}}, \mathrm{Bsn}^{\Delta \mathrm{Ex} 4 / 5}$ and wildtype littermate controls were found, indicating that vesicle replenishment of the smaller RRP was sufficient following the more physiological stimulation in vivo.

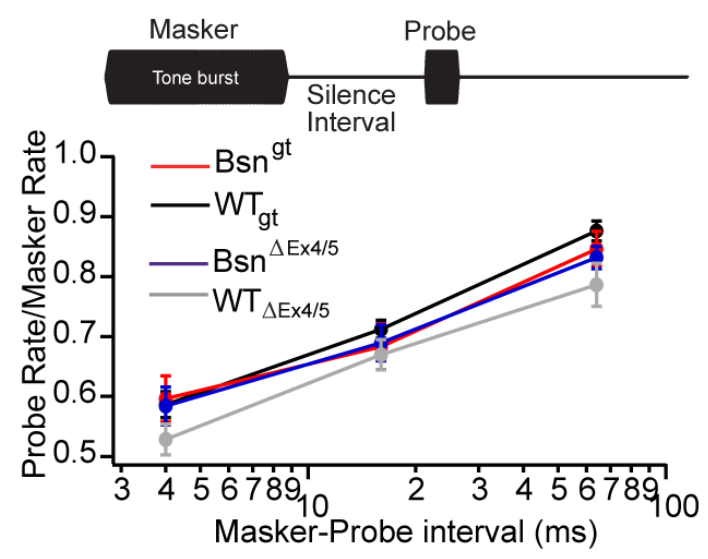

Figure 3.13: The time course of recovery from forward masking was similar in ANFs from all genotypes, indicating a normal time course of replenishment of the smaller synaptic vesicle pool. Top, illustration of the stimulus paradigm for the forward masking experiment: $100 \mathrm{~ms}$ masker tone burst and a $15 \mathrm{~ms}$ probe tone burst are separated by a silent interval of varying duration. Both tone bursts are presented at $\mathrm{CF}$, $30 \mathrm{~dB}$ above threshold. The duration of a complete cycle is $500 \mathrm{~ms}$. Bottom, the recovery time course of forward masking was presented as the ratio of firing rates in response to the $15 \mathrm{~ms}$ probe stimulus to the first $15 \mathrm{~ms}$ of the masker response. The time course of recovery from forward masking was similar in all genotypes $(n=29$ for $\mathrm{Bsn}^{\mathrm{gt}}, \mathrm{n}=16$ for $\mathrm{WT}_{\mathrm{gt}}, \mathrm{n}=17$ for $\mathrm{Bsn}^{\Delta \mathrm{Ex} 4 / 5}, \mathrm{n}=12$ for $\left.\mathrm{WT}_{\Delta \mathrm{Ex} 4 / 5}\right)$.

\subsubsection{Rate-level function and dynamic range}

Rate-intensity functions from both mutants are shown in Figure 3.14A. The reduced spike rate in both mutants was obvious also in this analysis. These rate-level functions had a shallower slope in both mutants and broader dynamic range. The increase in dynamic range was more pronounced in $\mathrm{Bsn}^{\mathrm{gt}}$, but a tendency was also found in $\mathrm{Bsn}^{\Delta \mathrm{Ex} 4 / 5}$ mice (Figure 3.14B). 

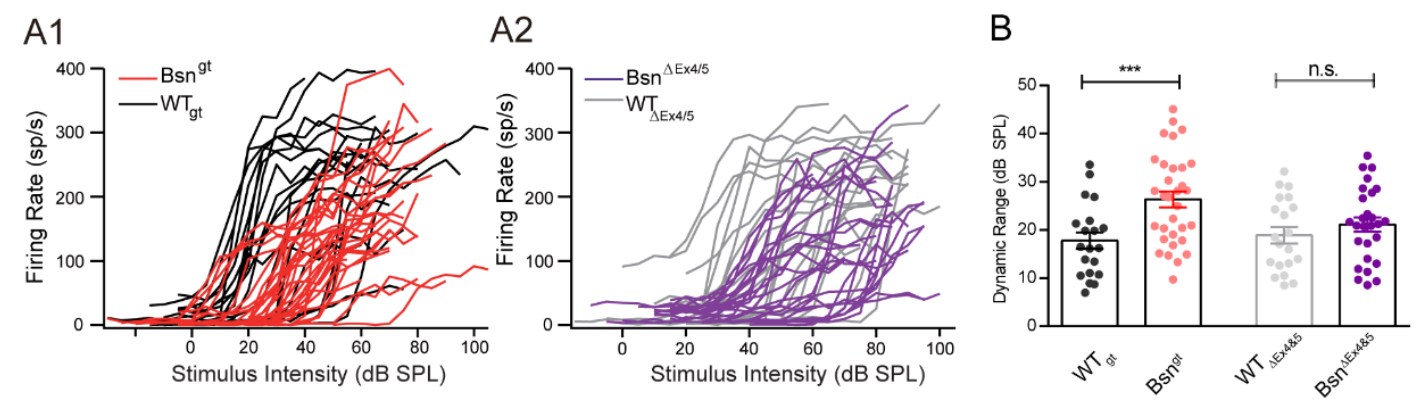

Figure 3.14: Rate-level function and dynamic range. (A) Rate-level functions of 20 $\mathrm{WT}_{\mathrm{gt}}$ (black), $32 \mathrm{Bsn}^{\mathrm{gt}}$ (red) (A1), $20 \mathrm{WT}_{\Delta \mathrm{Ex} 4 / 5}$ (grey) and $27 \mathrm{Bsn}^{\Delta \mathrm{Ex} 4 / 5}$ (violet) (A2) ANFs. (B) Dynamic ranges of $\mathrm{BSN}^{\mathrm{gt}}$ ANFs were broader compared to $\mathrm{WT}_{\mathrm{gt}}$ fibers $\left(26.3 \pm 1.7 \mathrm{~dB}\right.$ for $\mathrm{BSN}^{\mathrm{gt}} \mathrm{vs} .17 .8 \pm 1.7 \mathrm{~dB}$ for $\left.\mathrm{WT}_{\mathrm{gt}}, \mathrm{p}<0.001\right)$. A tendency was also found for $\mathrm{Bsn}^{\Delta \mathrm{Ex} 4 / 5}$ ANFs $\left(21.1 \pm 1.5 \mathrm{~dB}\right.$ for $\mathrm{Bsn}^{\Delta \mathrm{Ex} 4 / 5}$ vs. $18.9 \pm 1.7 \mathrm{~dB}$ for $\left.\mathrm{WT}_{\Delta \mathrm{Ex} 4 / 5}, \mathrm{p}=0.3\right)$.

\subsubsection{Phase locking to amplitude modulated tones}

Using amplitude modulated tones, the phase locking was measured using transposed CF tones, the amplitude of which is modulated by 125, 250, 500 and $1000 \mathrm{~Hz}$ at different amplitudes at $5 \mathrm{~dB}$ steps. Higher modulation frequencies cannot be followed because of the membrane time constant of IHCs low-pass filtering the response to the mechanoelectrical transducer current. Both mutants showed a similar ability to encode amplitude modulation when using SI as a measure of phase locking (Figure 3.15).

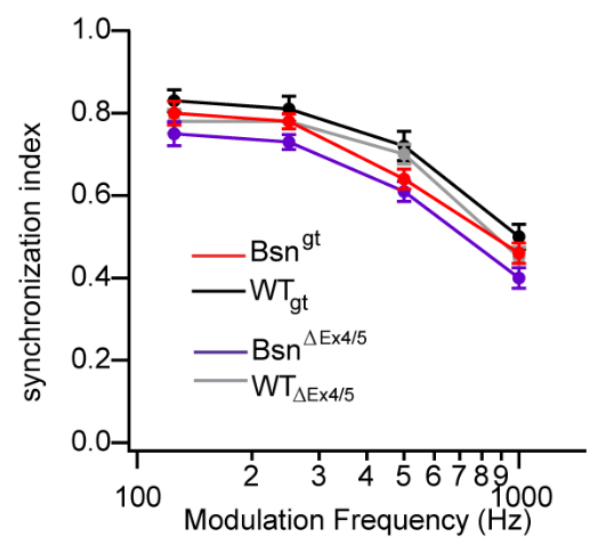

Figure 3.15: Average synchronization index as a function of modulation frequency. Both $\mathrm{Bsn}^{\mathrm{gt}}$ and $\mathrm{Bsn}^{\Delta \mathrm{Ex} 4 / 5}$ ANFs showed similar dependence for all four modulation frequencies without reaching significant. 
In summary, the single unit recording from ANFs did not support the segregation of responses in $\mathrm{Bsn}^{\mathrm{gt}}$ mice into two groups, which was otherwise predicted if the remaining looser anchored synaptic ribbon can support additional function in vivo.

\subsubsection{Auditory population responses in $\mathrm{Bsn}^{\mathrm{gt}}$ and $\mathrm{Bsn}^{\mathrm{AEx} 4 / 5}$ mice}

The single unit results above did not reveal a clear functional role of remaining Bsn ${ }^{\mathrm{gt}}$ ribbon-occupied synapses. However, it is still possible to reveal differences by assessing the population responses. We next analyzed auditory brainstem responses (ABR), of which wave I reflects the compound action potential of the ANFs (Melcher, Guinan et al. 1996, Melcher and Kiang 1996, Melcher, Knudson et al. 1996). The sound pressure levels evoking a reproducible threshold response were significantly elevated to a similar degree in both Bsn mutants when comparing to their respective littermate control (Figure 3.16A). $\mathrm{ABR}$ thresholds of $\mathrm{Bsn}^{\mathrm{gt}}$ mice were slightly elevated compared those of $\mathrm{Bsn}^{\Delta \mathrm{Ex} 4 / 5}$. This might have resulted from the different genetic backgrounds since a similar increase was also found for $\mathrm{WT}_{\mathrm{gt}}$ mice compared to $\mathrm{WT}_{\Delta \mathrm{Ex} 4 / 5}$ mice. In response to suprathreshold click stimulation ( $80 \mathrm{~dB}$ peak equivalent), the $\mathrm{ABR}$ waveforms were comparable in $\mathrm{Bsn}^{\mathrm{gt}}$ and $\mathrm{Bsn}^{\Delta \mathrm{Ex} 4 / 5}$ mutants (Figure 3.16B). Both waveforms showed a reduction of wave I amplitude, indicating a major reduction of synchronized activation of the ANFs. A small potential preceding $\mathrm{ABR}$ wave I, presumably representing the summating potential that primarily reflects the receptor potential of the IHCs, was unaffected in both mutants. We observed reduced amplitudes and a slight delay in latencies of all waves at all stimulus intensities. The amplitudes of central auditory responses (wave II and higher) were better

preserved, possibly reflecting some degree of compensation by convergence of auditory nerve fibers onto the neurons of the cochlear nucleus or homeostatic plasticity in the auditory brain stem nuclei. ABRs from all genotypes showed a comparable amplitude reduction when the click stimulus rate was increased from 20 to $100 \mathrm{~Hz}$ (data not shown). In summary, no significant differences were detected between two Bsn mutants at population response level as well. 

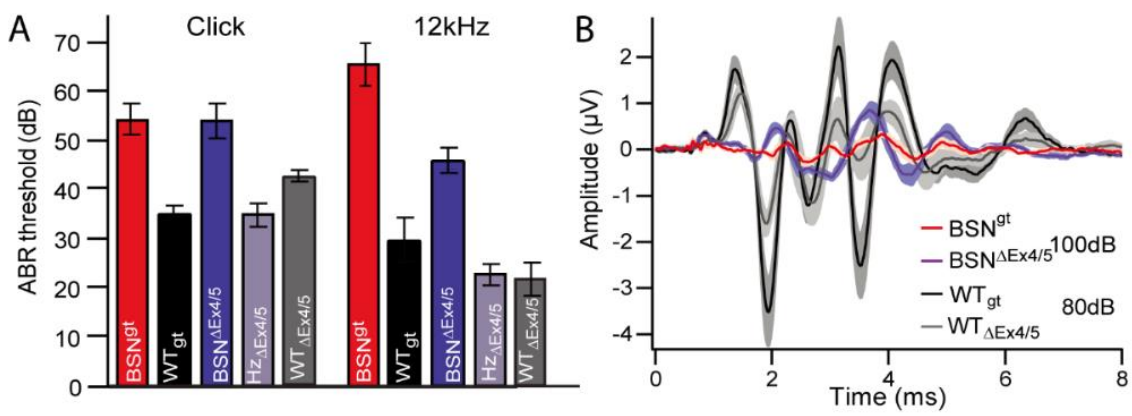

Figure 3.16: Auditory brainstem responses. (A) ABR thresholds for click and $12 \mathrm{kHz}$ tone burst stimuli were shifted to a comparable degree in $\mathrm{Bsn}^{\mathrm{gt}}$ and $\mathrm{Bsn}^{\Delta \mathrm{Ex} 4 / 5}$ animals compared to their respective littermate controls. The difference between $\mathrm{WT}_{\mathrm{gt}}$ and $\mathrm{WT}_{\triangle \mathrm{Ex} 4 / 5}$ was strain dependent. (B) Average of $\mathrm{ABR}$ waves in response to $100 \mathrm{~dB}$ (mutants) or $80 \mathrm{~dB}$ (wildtypes) click stimuli from $\mathrm{Bsn}^{\text {gt }}$ (red, $\mathrm{n}=7$ ) and $\mathrm{Bsn}^{\Delta \mathrm{Ex} 4 / 5}$ (purple, $\mathrm{n}=6$ ) mutants and $\mathrm{WT}_{\mathrm{gt}}$ (black, $\mathrm{n}=9$ ) and $\mathrm{WT}_{\Delta \mathrm{Ex} 4 / 5}$ (grey, $\mathrm{n}=3$ ) controls. The lack of ABR peak I wave may be explained by the reduced reliability of spiking activity. (B) The thresholds are elevated by $25 \mathrm{~dB}$ SPL in both mutants in response to $12 \mathrm{kHz}$ tone bursts and clicks. 


\subsection{Early onset progressive sensorineural hearing loss in Black Swiss Mice: auditory nerve responses in relation to underlying cochlear pathophysiology}

\subsubsection{Early onset hearing loss reflected in auditory population responses}

BLSW mice show early onset progressive hearing loss. To characterize the degradation of hearing, we first recorded the auditory brainstem responses from p14 and p21 BLSW as well as C57BL/6 control mice. Both p14 and p21 BLSW mice had significantly elevated thresholds (Figure 3.17A). If there was gain of function at p14 of ANF responses, we might be able to see that reflected in the amplitude of ABR wave I. When stimulated at high sound pressure levels, the wave I amplitude of BLSW mice grew steeper to a comparable size as in C57 control at p14, while the amplitude at p21 was much reduced (Figure 3.17B). A steeper amplitude growth function indicates abnormal recruitment of ANFs in the absence of compressive nonlinearity in the cochlea, for example, due to the lack of OHCs mechanical amplification. This observation suggested several possibilities in BLSW mice: (1) the dependence of discharge rate of ANFs on stimulus intensity is abnormally steep in at least p14 mice. (2) From p14 to p21, the synchronous spike activity and/or spike rate was reduced in ANFs. (3) The number of ANF was reduced.
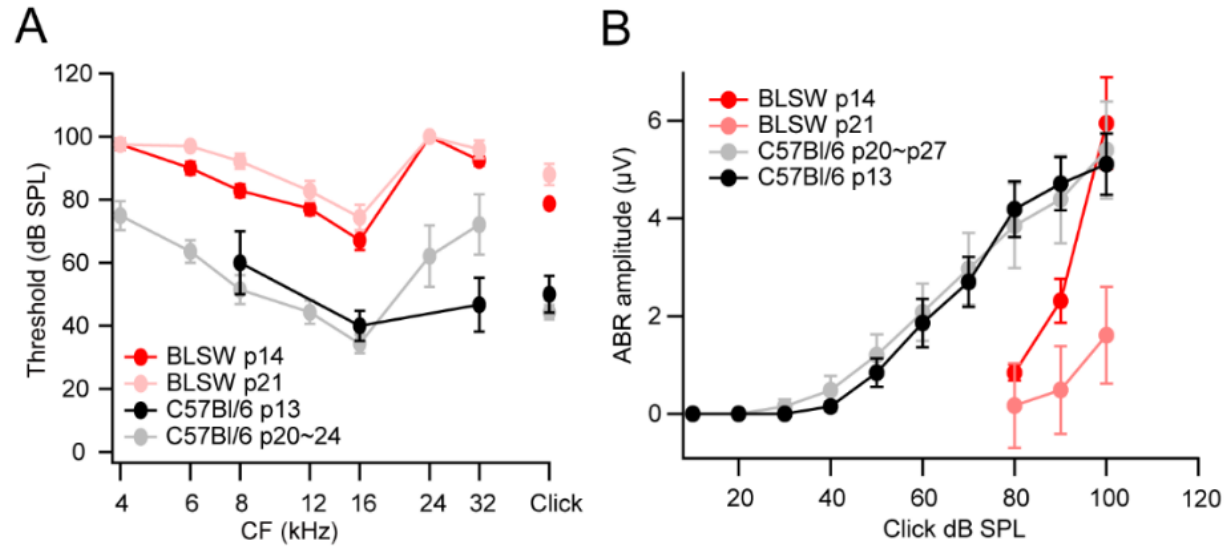

Figure 3.17: Auditory population responses in BLSW mice. (A) ABR thresholds for click and $12 \mathrm{kHz}$ tone burst stimuli were elevated in both p14 (n=9) and p21 (n=10) BLSW mice compared to C57BL/6 control ( $\mathrm{n}=4$ for $\mathrm{p} 13$; $\mathrm{n}=7$ for $\mathrm{p} 20-24)$. (B) Average ABR wave I amplitude as a function of click sound pressure level. In BLSW p14 mice $(n=11)$, higher click levels were needed to evoke a reproducible ABR wave I, but the amplitude of this wave can grow very steeply to a value which was comparable to C57BL/6 controls ( $n=4$ for $\mathrm{p} 13 ; \mathrm{n}=9$ for $\mathrm{p} 20-27)$. However, at $\mathrm{p} 21(\mathrm{n}=10)$, the wave I amplitude of BLSW mice did not reach p14 and control levels. 
To address the question if the synapse number is reduced in BLSW IHCs Dr. Sangyong Jung performed immunohistochemistry using antibodies against GluA2 and CtBP2 to mark postsynaptic and presynaptic structures, respectively. Synaptic ribbons were juxtaposed to GluA2 immunofluorescence clusters, suggesting a qualtitatively undisturbed ribbon anchorage in BLSW IHCs (Data not shown). Moreover, the number of synapses in the mid-apical region, measured by counting GluA2 immunofluorescence clusters, was comparable between BLSW and C57BL/6 IHCs in both p14 and p21 mice (Data not shown, Dr. Sangyong Jung). This observation is compatible with previous histological experiments, which revealed a normal morphology of the organ of Corti and the spiral ganglion neurons at 8-week-old BLSW mice, but a significant degenerating starting at the base and progressing towards the apex at 52-week-old mice (Charizopoulou, Lelli et al. 2011). Therefore, the reduction of ABR wave I amplitude at p21 in BLSW mice could not be attributed to degeneration of afferent innervation.

\subsubsection{ANF response properties and cochlear lesion in BLSW mice}

\subsubsection{Frequency tuning and threshold changes indicate an OHC amplification deficit in the BLSW cochlea}

Next, single ANF recordings were used to assess the ANF response properties in BLSW mice. A loss of amplification can lead to broader tuning curves and elevated threshold near CF (Kiang, Liberman et al. 1986). Figure 3.18A shows representative tuning curves of BLSW ANFs. Both p14-18 and p21-25 ANFs had significantly higher threshold at CF compared to C57BL/6 controls. There was an age dependent increase in the threshold from p14-18 to p21-25 (Figure 3.18B). Tuning curves were more broadly tuned compared to wildtype fibers. While there was an age dependent increase in the sharpness of tuning in C57BL/6 mice, the Q10 dB of tuning curves of BLSW fibers showed no age dependent change and was much smaller than that of controls. In summary, BLSW ANFs have higher single fiber threshold as well as lower frequency selectivity. The age dependent elevation in fiber threshold from p14-18 to p21-25 may explain the reduced ABR wave I amplitude because less fibers were recruited in response to the same stimulus level. 

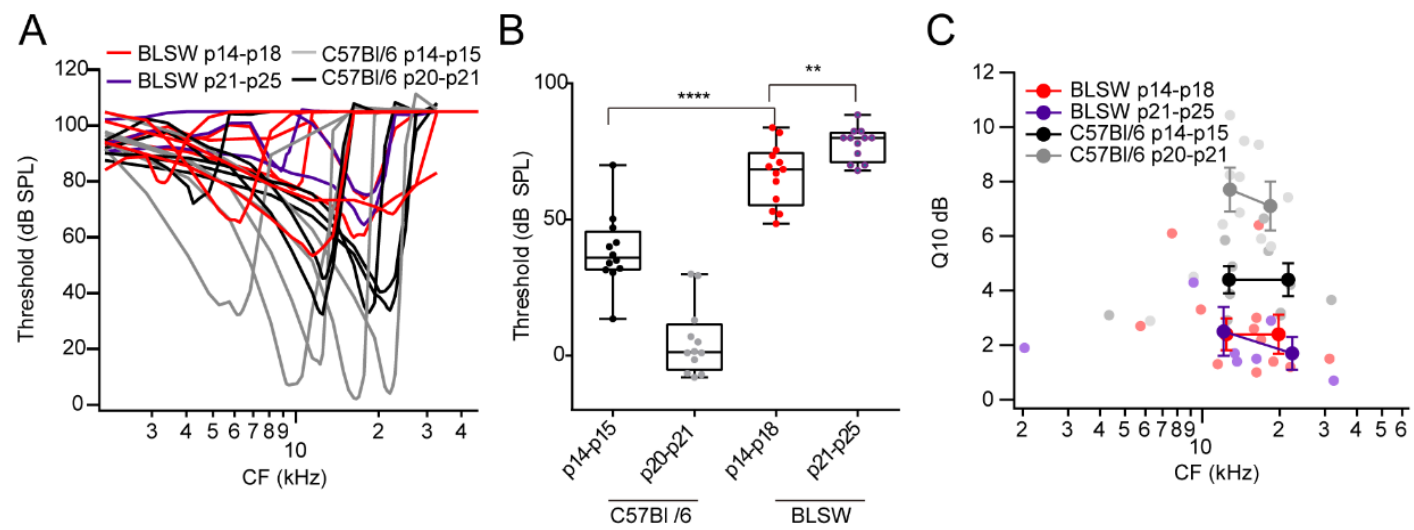

Figure 3.18: Tuning curve thresholds and frequency selectivity in BLSW ANFs. (A) Representative tuning curves of BLSW p14-18 (red) and p21-25 (blue) ANFs. (B) BLSW p14-18 (66.6 $\pm 3.1 \mathrm{~dB}, \mathrm{n}=13, \mathrm{p}<0.0001)$ and $\mathrm{p} 21-25(77.8 \pm 1.8 \mathrm{~dB}, \mathrm{n}=12)$ ANFs have significantly elevated thresholds at CF compared to C57BL/6 p14-15 (38.5 \pm 3.9 $\mathrm{dB}, \mathrm{n}=12)$ and $\mathrm{p} 20-21(5.4 \pm 3.7 \mathrm{~dB}, \mathrm{n}=12)$ fibers. (C) Sharpness of tuning was significantly broader in BLSW ANFs $(2.7 \pm 0.5, \mathrm{n}=12$ for BLSW p14-18 v.s. $4.3 \pm 0.4$, $\mathrm{n}=12$ for C57BL/6 p14-15, $\mathrm{p}<0.05 ; 2.1 \pm 0.5, \mathrm{n}=7$ for BLSW p21-25 v.s. $7.1 \pm 0.6$, $\mathrm{n}=12$ for $\mathrm{C} 57 \mathrm{BL} / 6 \mathrm{p} 20-21, \mathrm{p}<0.0001)$.

\subsubsection{Spontaneous discharge activity}

In the absence of sound stimuli, ANFs spontaneously discharge action potentials (Kiang 1965). The time pattern was highly irregular, Poisson-like events. Figure 3.19A shows representative inter-spike interval distribution from BLSW and C57BL/6 control ANFs. The inter-spike interval histogram in BLSW fibers showed a Poisson-like distribution, which was indistinguishable from that observed in wildtype control ANF when matched for the spontaneous discharge rate (Figure5.3A).

It has been shown that IHCs in BLSW cochlea have higher resting membrane potential (Charizopoulou, Lelli et al. 2011), which might lead to an increase in the spontaneous discharge rate. Consistent with this prediction, the spontaneous discharge rate distribution for fibers sampled in BLSW mice was shifted to larger rate compared to that of wildtype controls (Figure 3.19B). The elevation of single fiber threshold complicates the interpretation of this observation (Figure 3.18), because a subset of high threshold fibers may not be activated by up to $100 \mathrm{~dB}$ noise bursts. This might lead to an undersampling of a population of high threshold fibers with low spontaneous firing rates. 

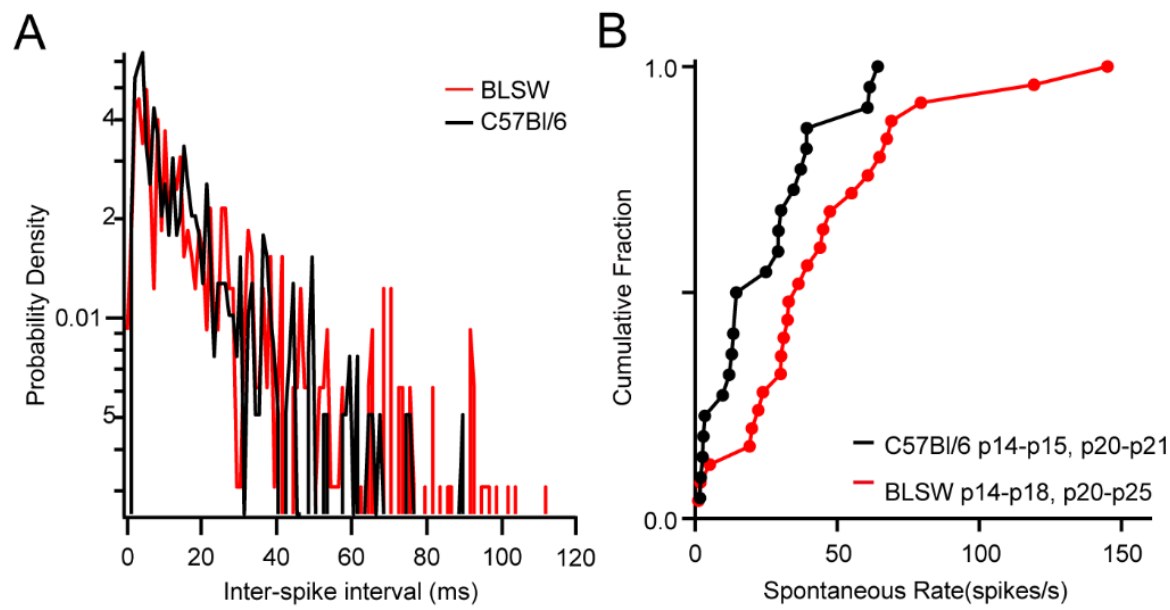

Figure 3.19: Spontaneous activity in BLSW ANFs. (A) Inter-spike interval distribution of two representative p15 ANFs with similar spontaneous firing rate from BLSW (red, $32.6 \mathrm{~Hz}$ ) and C57BL/6 (black, $40.9 \mathrm{~Hz}$ ). The typical Poisson-like distribution was retained in BLSW ANFs. (B) Cumulative histogram of spontaneous rate distribution. On average, ANFs of BLSW mice had significantly higher spontaneous discharge rate $(45.0 \pm 6.7 \mathrm{~Hz}, \mathrm{n}=25$ for BLSW p14-18, p20-25 pool; 23.5 $\pm 4.0 \mathrm{~Hz}, \mathrm{n}=24$ for $\mathrm{C} 57 \mathrm{BL} / 6 \mathrm{p} 14-15$, p20-21 pool; $\mathrm{p}<0.01$ )

\subsubsection{Rate-level functions and dynamic range in BLSW ANFs}

The mechanical amplification of OHCs enables a broad dynamic range of ANFs by nonlinearly compression of the input signal into more gradually basilar membrane vibrations (Yates 1990, Yates, Winter et al. 1990). Lack of amplification might lead to a loss of this compressive nonlinearity, so that the rate-level functions of ANFs might raise more steeply than those of control ANFs.

Representative rate-level functions of BLSW and C57BL/6 ANFs are illustrated in Figure 3.20A. Rate-level functions were constructed from average rates in response to tone bursts of different levels at CF. The elevated threshold at CF was also obvious. Ratelevel functions were fitted with a five parameters models (Chapter 2) to better approximate dynamic range. As shown in Figure 3.20B, dynamic ranges in BLSW fibers were significantly narrower than those in C57BL/6. In summary, rate-level functions of BLSW ANFs have much steeper growth slope compared to that of wildtype controls. This is consistent with an abnormal OHCs mechanical amplification function as early as p14. 

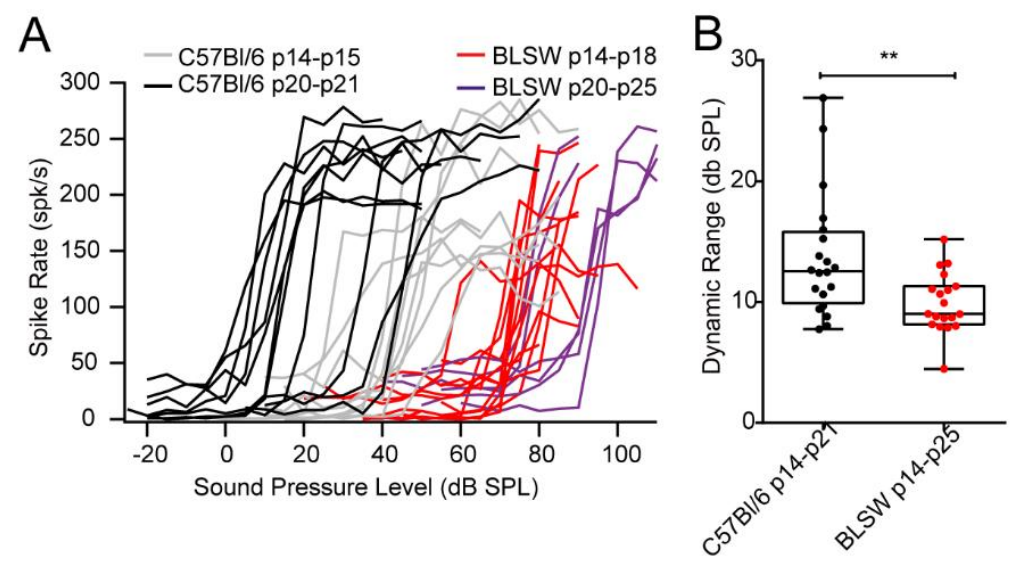

Figure 3.20: Rate-level functions and dynamic range for BLSW and C57BL/6 ANFs. (A) Representative rate-level functions of different age groups. (B) Narrower overall dynamic range in BLSW ANFs compared to those in C57BL/6 $(9.9 \pm 0.6 \mathrm{~dB}, \mathrm{n}=19$ for BLSW ANFs; $13.7 \pm 1.1 \mathrm{~dB}, \mathrm{n}=20$ for $\mathrm{C} 57 \mathrm{BL} / 6, \mathrm{p}<0.01$ ).

\subsubsection{Discharge rate and adaptation to suprathreshold tone bursts}

The maximum ABR wave I amplitude in p14 BLSW mice can be as large as wildtype level, but not in p21 mice (Figure 3.17B). BLSW mice showed high susceptibility to audiogenic seizures at p14, which was suggested to be correlated with an increased wave I amplitude at that age (Charizopoulou, Lelli et al. 2011). Therefore, the evoked responses from animals aged around p14 and p21 were compared. BSLWs ANFs tended to have shorter median first spike latency at p14-18 (Figure 3.20B) without reaching significance. The onset rate was significantly larger in BLSW ANFs (Figure 3.20C) and the adapted rate showed a tendency to be increased but did not differ significantly from that of C57BL/6 fibers. At the age of p21-25, BLSW ANFs fired at a rate and latency comparable to that of both BLSW p14-18 and C57BL/6 ANFs, arguing against the reduced amplitude at p21 BLSW mice resulted from reduced spike rate and spike synchrony (Figure 3.21 C, D).

To assess the adaptation time course, which most likely reflect depletion of presynaptic RRP of vesicles (Moser and Beutner 2000, Goutman and Glowatzki 2007) a double exponential function was fitted to the PSTH (Westerman and Smith 1984). Both BLSW and C57BL/6 ANFs showed virtually identical rapid $(0.32 \pm 0.065 \mathrm{~ms}, \mathrm{n}=25$ for BLSW ANFs; $0.28 \pm 0.062 \mathrm{~ms}, \mathrm{n}=24$ for $\mathrm{C} 57 \mathrm{BL} / 6 \mathrm{ANFs}$ ) and short term adaptation time 
constants $(10.3 \pm 1.1 \mathrm{~ms}, \mathrm{n}=25$ for BLSW ANFs; $11.8 \pm 1.1 \mathrm{~ms}, \mathrm{n}=24$ for C57BL/6 ANFs $)$, with no age dependence (data not shown).
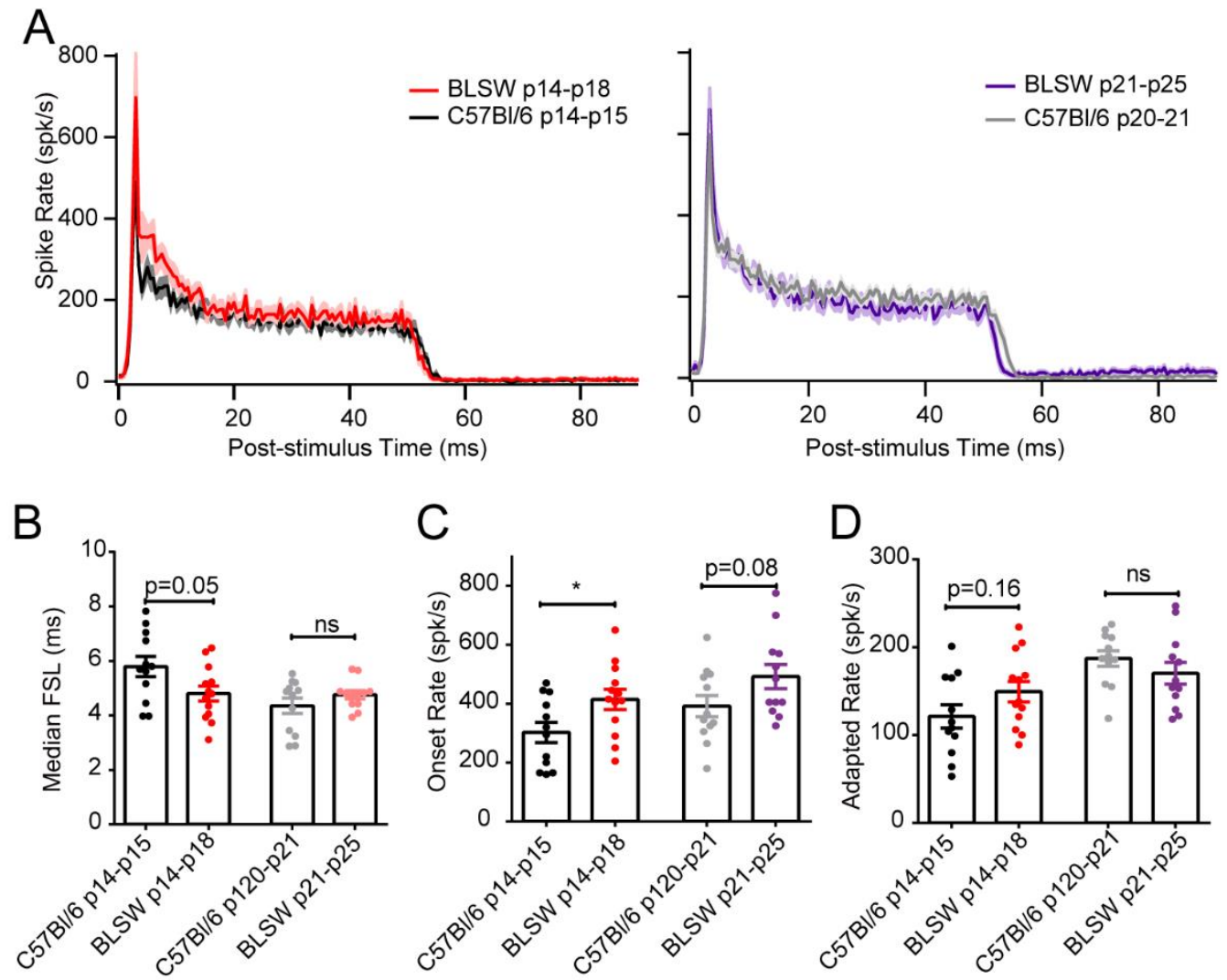

Figure 3.21: Evoked responses of BLSW and C57BL/6 ANFs to $50 \mathrm{~ms}$ superathreshold tone bursts at CF. (A) Average post-stimulus time histogram of 13 BLSW p14-18 (red, left), 12 C57BL/6 p14-15 (black, left), 12 BLSW p21-25 (purple, right) and 12 C57BL/6 p20-21 (gray, right) ANFs. PSTHs were aligned to the bins of their respective median first spike latency before averaging. (B) Median first spike latencies of the fibers in (A). BLSW p14-18 fibers tended to have shorter latency in comparison to p14-15 C57BL/6 fibers. However, the difference did not reach significance. (C) Significantly larger onset firing rate (average firing rate over $1 \mathrm{~ms}$ window after median first spike latency) for BLSW p14-18 ANFs compared to C57BL/6 p14-15 (414.2 $\pm 33.9 \mathrm{~Hz}$ for BLSW p14-18; $302.5 \pm 34.2 \mathrm{~Hz}$ for C57BL/6 $\mathrm{p} 14-15, \mathrm{p}<0.05)$. There was a tendency for larger onset rates of BLSW ANFs at 3-4 weeks $(491.7 \pm 40.9 \mathrm{~Hz}$ for BLSW p21-25; $391.7 \pm 36.2 \mathrm{~Hz}$ for C57BL/6 p20-21, $\mathrm{p}=0.06$ ). (D) Analysis of adapted rate (average firing rate over the last $5 \mathrm{~ms}$ window of tone bursts after median first spike latency). Adapted firing rates did not differ significantly in both age groups. 
Next, the recovery time course from adaptation was studied using a forward masking paradigm. The masker elicits rapid adaptation, which can be attributed to depletion of presynaptic RRP of vesicles. The recovery was revealed by using a probe stimulus of identical intensity following masker tone with different intervals (Figure 3.22A). A similar time course of recovery was found in BLSW and C57BL/6 fibers, indicating that vesicle replenishment was not affected in BLSW IHCs.

A

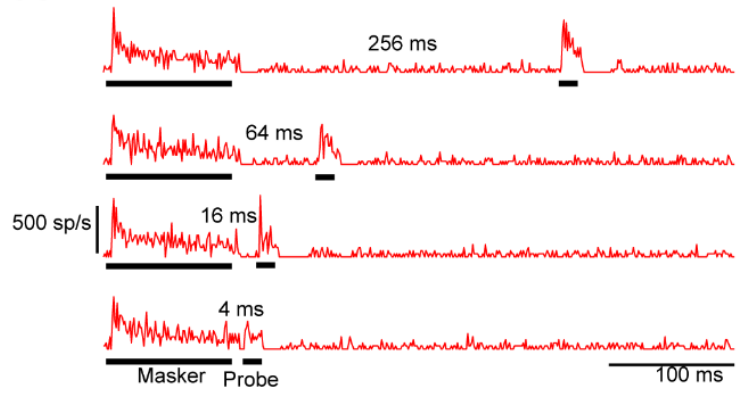

B

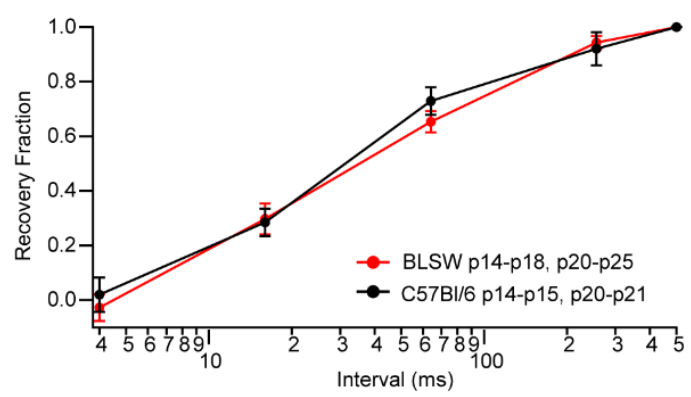

Figure 3.22: Recovery from adaptation assessed using a forward-masking paradigm. (A) Representative responses of a BLSW ANF (p22) to the experimental paradigm. (B) Similar time course of recovery from forward masking in BLSW $(n=9)$ and C57BL/6 $(\mathrm{n}=8)$ ANFs. Firing rates in response to $15 \mathrm{~ms}$ probe stimulus was normalized to the last $15 \mathrm{~ms}$ (0\% recovery) and first $15 \mathrm{~ms}$ of masker response (100\% recovery).

\subsubsection{Unaltered phase locking to amplitude-modulated tones}

Transposed tones ( $\mathrm{CF}$ carrier frequency continuous tones which were amplitudemodulated by a half wave rectified modulator sinusoid) with modulation frequencies of $125 \mathrm{~Hz}, 250 \mathrm{~Hz}, 500 \mathrm{~Hz}$ and $1 \mathrm{kHz}$ were used to measure the ability of ANFs to encode amplitude modulated tones. The receptor potential of IHCs was modulated using this paradigm. As the modulation frequency increases, the membrane potentials cannot follow the modulation because of membrane time constant, which leads to decreased ability of phase locking to amplitude modulation. BLSW ANFs showed similar ability to synchrony with those amplitude-modulated tones (Figure 3.23). This result suggests that BLSW IHCs can still encode temporal information using graded membrane potential. Therefore, IHCs retained the ability to transduce mechanical vibration into proper membrane 
potential even with the presence of abnormal stereocilia morphology (Charizopoulou, Lelli et al. 2011). This, as well as the shorter first spike latency also argues against an increased membrane time constant as it might be expected given the reported reduction of the IHC potassium conductance.

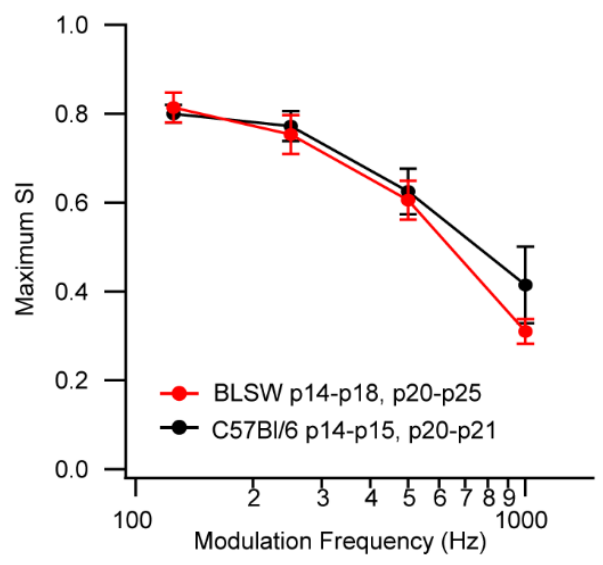

Figure 3.23: Average maximum synchronization index (SI) as a function of modulation frequency of $15 \mathrm{BLSW}$ and 7 C57BL/6 ANFs.

\subsubsection{IHC cell physiology in BLSW mice}

Previous results from single fiber recordings support the notion of a lack of OHC mechanical amplification (Charizopoulou, Lelli et al. 2011), which possibily results from a reduction in maximum transduction current amplitude in OHCs. Moreover, a similar reduction in maximum transduction current was also observed at p12 in BLSW IHCs (Charizopoulou, Lelli et al. 2011). A reduced maximum transduction current in IHC would predict a smaller receptor potential, therefore a smaller voltage dependent neurotransmitter release. However, single ANF recording did not show signs of reduced neurotransmitter release. To further understand ths discrepancy, additional cell physilogy experiments were performed by Dr. Sangyong Jung. BLSW IHCs aged between p14 and p21 showed significantly larger maximum $\mathrm{Ca}^{2+}$ current, and a hyperpolarizing shift of the voltage-dependence of $\mathrm{Ca}^{2+}$ channels compared to wildtype IHCs. In response to depolarization that evoked maximum $\mathrm{Ca}^{2+}$ current of different durations, BLSW IHCs showed significantly larger fast and sustained exocytosis. The fast component of exocytosis was interpreted as the size of readily releasable pool (RRP), while the sustained component mainly reflects vesicle replenishment. 
The larger sustained exocytosis in vitro suggests a possible gain of neurotransmitter release in vivo, thus a gain of sustained spike rate in ANFs. Indeed, previous single finber recording results showed significantly larger onset response and a tendency of larger adpated response in p14-18 ANFs. These observations could be explained by (1) reduction in maximum transduction currents in vivo condition, and (2) a gain of function of $\mathrm{Ca}^{2+}$ influx upon smaller transduction current, triggered comparable or even larger neurotransmitter release. The larger sustained exocytosis may reflect an abnormal recycling of cell membrane or exocytosis that happens outside of AZs. 


\subsection{Optical stimulation of ChR2 expressing ANFs: endeavor towards rescuing hearing by light}

\subsubsection{Optogenetic stimulation of ChR2 mice cochlea}

In collaboration with Dr. Victor Hernandez, we first studied the expression pattern of ChR2 in cochlea of the transgenic mice. Here, ChR2 is coupled to YFP and the expression is controlled under the Thy-1.2 promoter. ChR2-YFP signal was detected in the somata as well as in the peripheral and central axons of most spiral ganglion neurons (SGN), but not in hair cells. 4-10-week-old ChR2 mice were used for the subsequent studies as the expression of ChR2 started from the end of the $3^{\text {rd }}$ postnatal week (data not shown).

A $473 \mathrm{~nm}$ continuous wave laser coupled via a $250 \mu \mathrm{m}$ optical fiber was inserted into the cochlea via a small cochleostomy. Figure 3.24 shows an example traces of optical auditory brainstem responses (oABR) evoked by laser stimulation. The oABR reflect optical evoked population responses of neurons, which can be inhibited by tetrodotoxin application to the round window. To better understanding the optical evoke response of individual ANF and cochlear nucleus neurons, single unit recordings were subsequently performed. In three successful experiments, nine neurons responded to optogenetic stimulation (Figure 3.24). Because acoustically evoked responses were not available for those neurons, an unambiguous classification of unit into ANF or cochlear nucleus could not be performed. These neurons most likely represent a mixture of ANFs and cochlear nucleus neurons. Light responsive neurons typically fired one or two action potential(s) in response to $5 / 10 \mathrm{~ms}$ laser pulses, and only two neurons fires multiple spikes using 10 $\mathrm{ms} / 30 \mathrm{~ms}$ laser pulse stimulation. One of the neurons fired spontaneously. Six neurons responded with a relatively short first spike latency at stimulus onset $(4.8 \pm 0.3 \mathrm{~ms}$ from light onset, excluding one neuron with spontaneous firing: $4.6 \pm 0.3 \mathrm{~ms}$ ). Given such short latency, it is tempting to suggest these neurons most likely represent ANFs or globular bushy cells, which receive calyx type innervation from ANF. The trial-to trial variance of the first spike timing was very small for this subpopulation $\left(0.08 \pm 0.06 \mathrm{~ms}^{2}\right.$ without vs. $0.40 \pm 0.03 \mathrm{~ms}$ with inclusion of the spontaneously firing neuron). For comparison, responses of ANFs to acoustic clicks obtained in another set of experiment on C57BL/6 mice (From Dr. Nicola Strenzke) showed an average first spike latency of $3.2 \pm 0.9 \mathrm{~ms}$ ( $p<0.01$ compared to optogenetic stimulation, including the spontaneously firing neuron), 
and a mean trial-to-trial variance of $0.07 \pm 1.00 \mathrm{~ms}^{2}$ ( $\mathrm{p}<0.02$ compared to optogenetic stimulation, including the spontaneously firing neuron). Three other neurons had a longer $(13.2 \pm 1.6 \mathrm{~ms})$ and more variable $\left(2.40 \pm 0.90 \mathrm{~ms}^{2}\right)$ first spike latency and likely represent cochlear nucleus neurons.
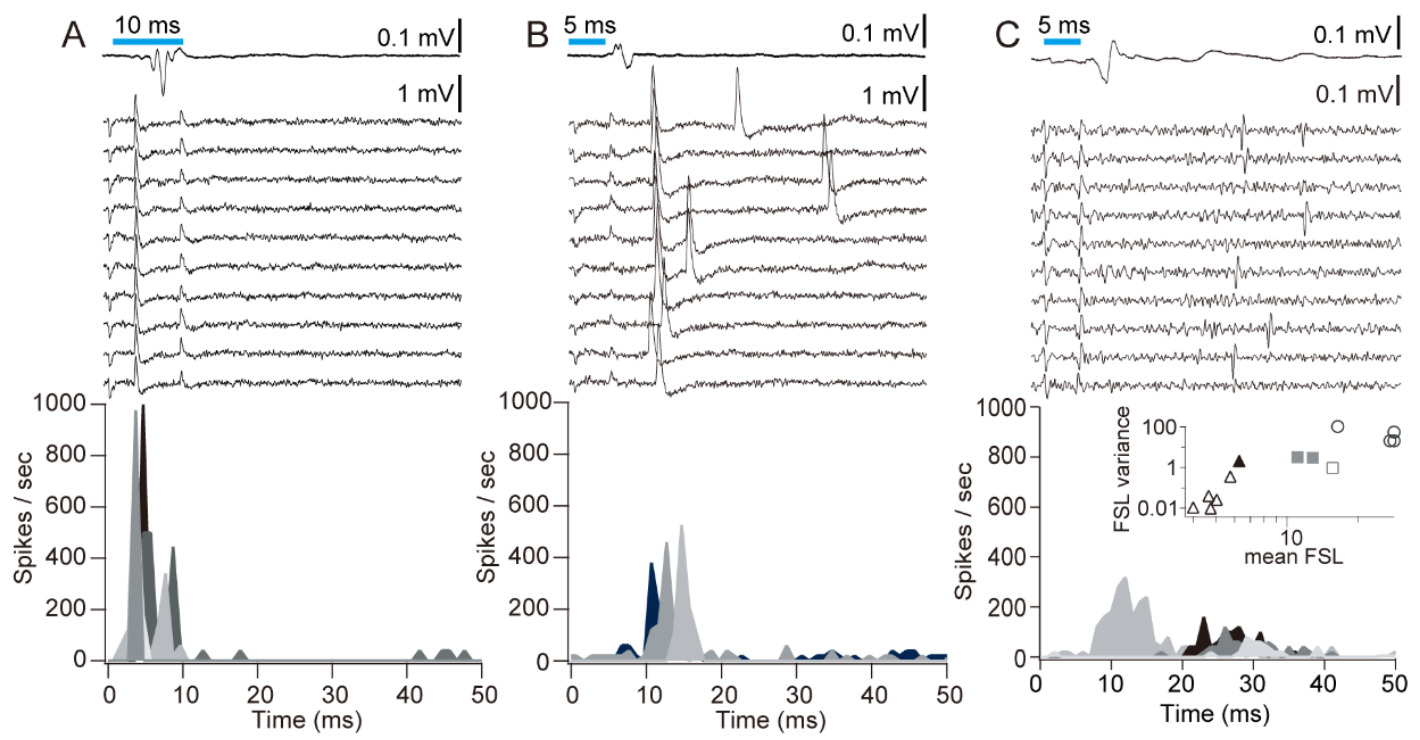

Figure 3.24: Microelectrode recordings from individual neurons of the auditory pathway in response to optogenetic stimulation. (A) Upper: simultaneously recorded oABR; Middle: relatively short latencies and remarkably high reliability and temporal precision of single neuron spiking near the internal auditory canal, presumably representing ANFs in response to intracochlear laser light stimulation; Bottom: poststimulus-time histogram (PSTH) of several neurons, distinguished by greyscale values. (B) Same for a different population of single neurons encountered within the cochlear nucleus, showing slightly longer latencies, lower reliability and increased jitter. (C) Same for a representative tungsten microelectrode recording from a neuron of the central nucleus of the inferior colliculus (IC), demonstrating delayed responses with reduced temporal precision. Insect: variance and average of the first spike latency of the neurons analyzed in a-c. Filled symbols represent units with spontaneous activity, typically associated with an increased variance of the first spike latency (IC recordings were performed by Dr. Victor H. Hernandez). 


\subsubsection{AAV6 mediated expression of Catch variant in SGNs and light-evoked responses}

The application of optogenetic stimulation requires expression of $\mathrm{ChR} 2$ in spiral ganglions (SGNs). One promising method is to use virus-mediated transduction of SGNs. Adeno-associated viruses (AAV) have been shown to transduce murine SGNs without compromising hearing. In collaboration with Dr.Victor H. Hernandez and Dr. Kristen Reuter, we performed transuterine AAV6 virus injections into the left otocyst of 8-14 embryos at 11.5 days post coitum amd achieved reliable and selective transduction of SGNs. Another ChR2 variant CatCh was chosen for this study because it shows higher $\mathrm{Ca}^{2+}$ permeability and was reported to provide for a 70 fold higher light sensitivity of neurons compared to wild-type ChR2 (Kleinlogel, Feldbauer et al. 2011). CatCh was fused to YFP. Immunocytochemistry data showed that CatCh-YFP was expressed in the membrane of SGN somata as well as their neurites contacting IHCs. Expression persisted at least up to postnatal day 59 with no obvious decay of expression level, and the expression was most abundant in the base of the cochlea (data not shown).

Next, single unit recording was performed using injected mice. Here, the optical fiber was inserted into the round window mainly because of the expression pattern of Catch in the base of the cochlea. Fourteen light responsive neurons were detected in two successful experiments (one neuron was excluded from analysis because the small spike amplitude hindered precise analysis of spike rate and timing). The short recording duration (10 ms) during those two experiments did not allow recording from neurons with long response latencies (>9 ms, Figure 3.25A). Firing of CatCh-expressing neurons required less laser power than needed in ChR2 transgenic mice (data not shown). The mean first spike latency in response to maximum laser power was found to be shorter than those of putative ANFs/bushy cells in the ChR2 mice $(2.8 \pm 0.2 \mathrm{~ms}, \mathrm{n}=9$ in the CatCh mice vs. 4.8 $\pm 0.3 \mathrm{~ms}, \mathrm{n}=6$ in ChR2 mice, $\mathrm{p}<0.001)$ and with very small trial-to trial variance $(0.02 \pm$ $0.01 \mathrm{~ms}^{2}$ ). The response latency and precision depended on stimulus intensity (Figure 3.25B). The evoked spikes were more reliable and precise with shorter latency when light power was increased (Figure 3.25B). 
A
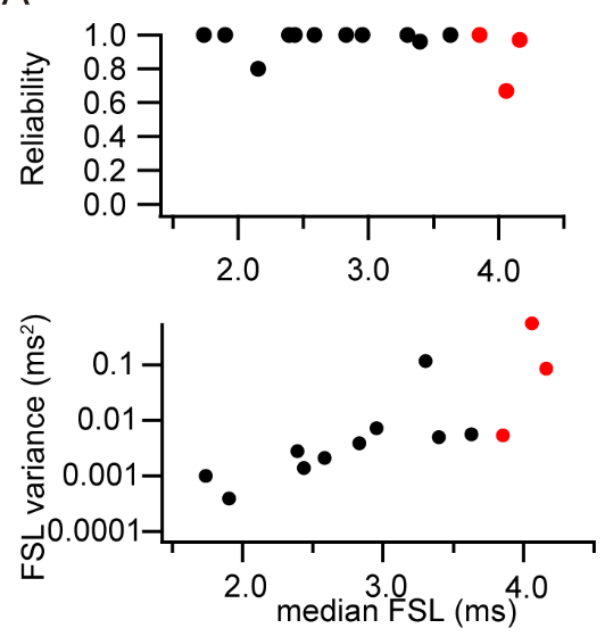

B
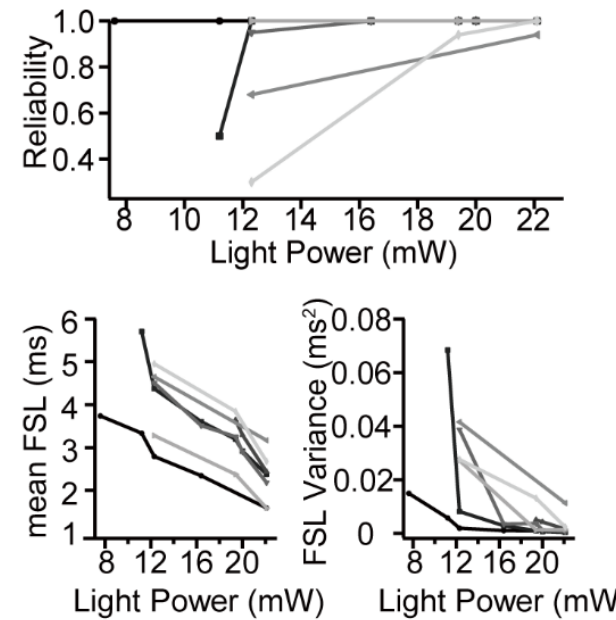

Figure 3.25: Analysis of responses from single neurons in Catch-transfected mice by optogenetic stimulation. (A) Reliability and precision of first spikes as a function of median first spike latency (FSL) in response to 5-10 ms maximum laser power stimulation. Red dots indicate three neurons, on which additional acoustic stimulation experiments were performed (Figure 3.26) .Top: Reliability is the proportion of trials eliciting a spike during the $1 \mathrm{~ms}$ bin centered at median FSL. Bottom: FSL variance in response to the same stimuli. (B) Quantification of the first spike latency (indicated by arrow in (A): Top: reliability of spiking (proportion of trials eliciting a spike during the time of window of stimulation); Bottom left: mean FSLs; Bottom right: FSL variance in response to 5 10 ms flashes at different light intensities. Greyscale values indicate 7 different light-responsive neurons near the internal auditory canal.

To test if the recorded neurons are from auditory nerve/cochlear nucleus, additional acoustic stimulations were applied to four units. Three of them showed responses to both noise/tone bursts (Figure 3.25 red dots). Figure 3.26A,B shows acoustic and optical evoked responses of two neurons. Both neurons have $\mathrm{CF}$ between 10 to $16 \mathrm{kHz}$ (Figure 3.26C, D). The short recording duration of $10 \mathrm{~ms}$ did not allow PSTH based unit classification. However, the neuron in Figure 3.26 A, C have a precise onset response followed by a silent interval about $1 \mathrm{~ms}$, which is a typical pattern seen from globular bushy cell in the cochlear nucleus (Blackburn and Sachs 1989, Taberner and Liberman 2005). Another neuron showed less clear response pattern for robust classification (Figure 3.26B), probably because $125 \mathrm{~dB}$ tone bursts failed to drive the neuron to maximum firing rate due to cochlear damage after cochleostomy. 
A Optical Stimulation $\stackrel{\Xi}{\longleftarrow}$

Acoustic Stimulation

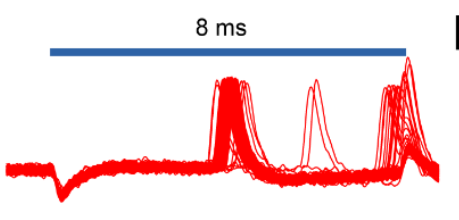

B
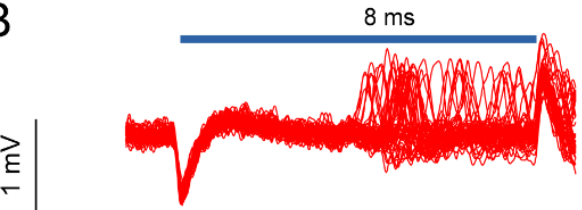

$125 \mathrm{~dB}$ Tone bursts

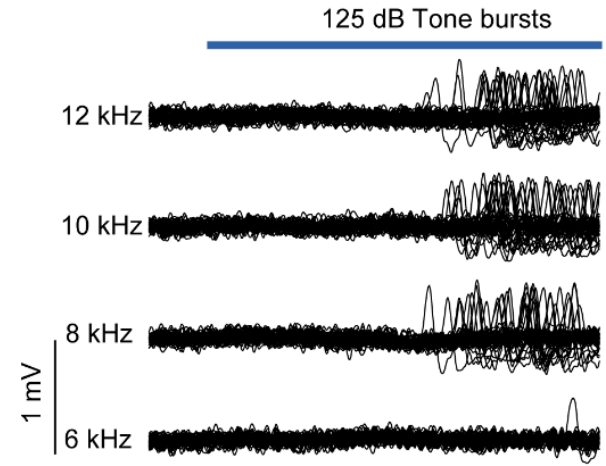

C
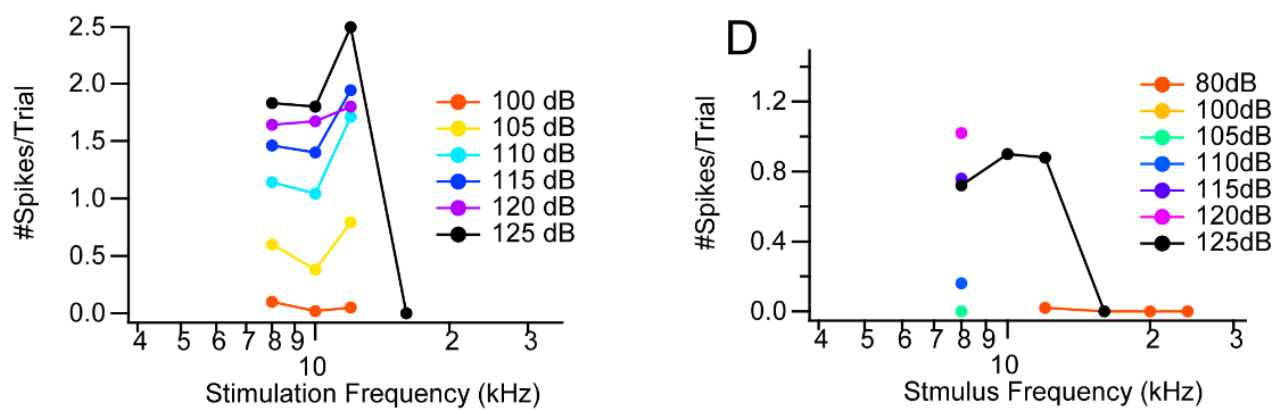

Figure 3.26: Acoustic and optogenetic stimulation confirmed the activation of neurons in the auditory pathway. (A, B) Top: Representative traces showing two neurons' responses to optical stimulation. Bottom: corresponding acoustic evoked response to $125 \mathrm{~dB}$ tone bursts at different stimulus frequencies. (C, D) Responses of neurons corresponding to (A, B) to tones of different frequencies and SPLs. For the neuron in (A), $100 \mathrm{~dB}$ tone bursts at $8-, 10-$ and $16 \mathrm{kHz}$ failed to elicit a change in number of evoked spikes, while the same $125 \mathrm{~dB}$ tone evoked a larger response at 12 $\mathrm{kHz}$. A similar pattern was observed in another neuron (B).

ANFs are capable of firing at a sustained rate at about $300 \mathrm{~Hz}$ during stimulation (Kiang 1965). The next question is if optogenetic stimulation can drive a fiber firing at that rate. Here, two different experimental paradigms were used. In the first paradigm, a 5-8 ms laser pulse in $10 \mathrm{~ms}$ trial was applied to each single fiber at different stimulus rate. Figure 3.27A shows a representative fiber's responses at different stimulation rates. This fiber was capable to follow stimulation at least up to $60 \mathrm{~Hz}$. As the repetition rate increased, the response latencies as well as the variance of FSL increased (Figure 3.27B left and middle). Only two units did not show any spikes during $1 \mathrm{~ms}$ bin centered at median FSL 
when stimulated with a repetition rate above 20 and $40 \mathrm{~Hz}$, respectively (Figure 3.27B right). For technical reasons, the paradigm described above cannot stimulate at a rate above $100 \mathrm{~Hz}$. Therefore, another pulse train protocol was designed to drive the laser pulse at a higher rate. Two light responding neurons were identified in additional two CatCh-AAV injected animals. Figure 3.27C shows one neuron's response to $3 \mathrm{~ms}$ pulse train stimulation. At stimulus rate of $125-$ and $200 \mathrm{~Hz}$, the neuron fired evoked spikes only in the first few pulses (Figure 3.27C). Another neurons, which responded with long median first spike latency $(13 \mathrm{~ms})$, fired only one spike in response to the first $5 \mathrm{~ms}$ light pulse in each trial at stimulus rate of $100 \mathrm{~Hz}$. Thus, 3 or $5 \mathrm{~ms}$ maximum laser power stimulation failed to continuously drive those two neurons to a firing rate above $100 \mathrm{~Hz}$. Future works are needed to address the question whether shorter latency or reduced laser power might be able to drive ANFs or cochlear nucleus neurons to fire at a higher sustained rates.

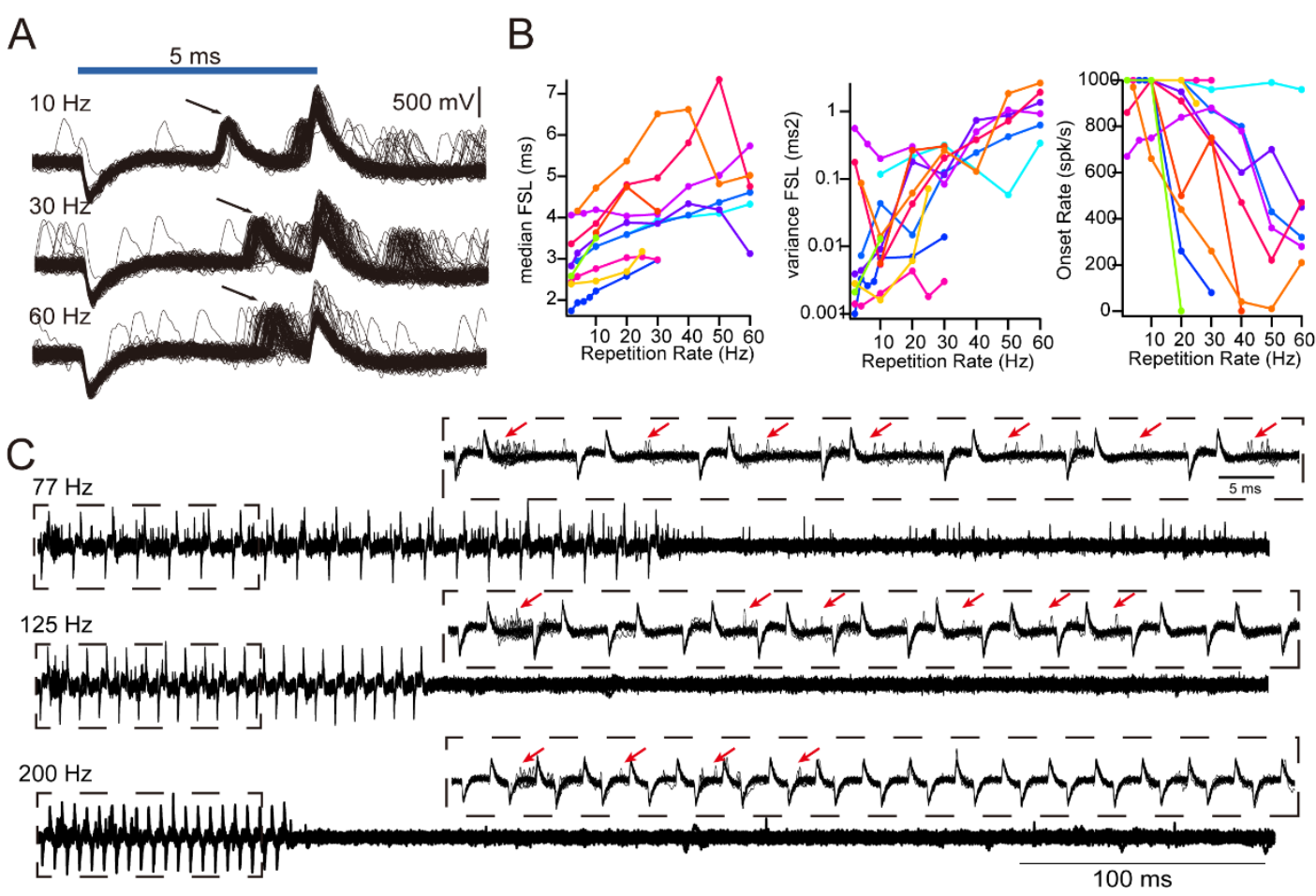

Figure 3.27: Responses of neurons to different stimulus rates. (A) Stimulation with 5 ms light pulses elicits precise and reliable spiking in a representative ANF in a CatChtransfected mouse at stimulus rate up to at least $60 \mathrm{~Hz}$. (B) Quantification of response properties as a function of repetition rate of ten light responding neurons to 5-8 $\mathrm{ms}$ long laser stimulation. Left: median first spike latency; Middle: first spike latency variance; Right: Onset rate in $1 \mathrm{~ms}$ bin centered at median first spike latency. (C) Responses to 3 
ms light pulse train separated by different intervals $(10 \mathrm{~ms}, 5 \mathrm{~ms}$ and $2 \mathrm{~ms}$ for $77 \mathrm{~Hz}$, $125 \mathrm{~Hz}$ and $200 \mathrm{~Hz}$, respectively) in another neuron. Each trial lasts $500 \mathrm{~ms}$, and contains 20 light pulses. Responses of 20 trials were plotted on top of each other. Red arrow indicate cluster of spikes in response to light pulse. The number of cycles having evoked spikes decreased as stimulus rate increased. 


\section{Discussions}

\subsection{Auditory nerve fiber response properties during early postnatal development}

During normal postnatal development in mice, several development changes occur in the peripheral auditory system. In this study, single fiber responding properties were characterized during the period of early postnatal age in mice. Before hearing onset (p12), spontaneous bursting activity was recorded from ANFs/cochlear nucleus neurons, which were still not responsive to tone or noise stimuli. After onset of hearing, several properties were found to further develop from p14-15 to p20-21 mice, including threshold, frequency selectivity and maximum evoked discharge rate to tone bursts at $\mathrm{CF}$.

\subsubsection{Development of spontaneous activity}

\subsubsection{Presynaptic mechanisms}

Spontaneous activity has been observed during the development of several mammalian sensory systems (Blankenship and Feller 2010). This activity in the absence of sensory input is thought to be important for the development of sensory neurons and their synaptic connections (Blankenship and Feller 2010, Bulankina and Moser 2012). Bursting activity has been observed for developing auditory system of several species (Eggermont, Smith et al. 1993, Gummer and Mark 1994, Lippe 1994, Tritsch, Yi et al. 2007). The patterned pre-sensory activity has been attributed to the pulsatile $\mathrm{Ca}^{2+}$ action potential (AP) generated by IHCs, triggering a burst of glutamate release (Beutner and Moser 2001), which in turn drives a burst of action potentials in ANF (Tritsch, Yi et al. 2007, Tritsch, Rodriguez-Contreras et al. 2010, Johnson, Kennedy et al. 2012). It has been shown that both purinergic signaling from supporting cells (Tritsch, Yi et al. 2007, Tritsch, Rodriguez-Contreras et al. 2010) and IHC transduction current (Johnson, Kennedy et al. 2012) may underlie the generation of IHC spontaneous action potentials.

$\mathrm{Ca}^{2+}$ influx through Cav1.3 channels is essential for neurotransmission between IHCs and afferent fibers (Platzer, Engel et al. 2000, Brandt, Striessnig et al. 2003). Recently, robust synaptic $\mathrm{Ca}^{2+}$ influx was observed using $\mathrm{Ca}^{2+}$ imaging techniques before hearing onset 
(Wong et al., 2013). During postnatal development before hearing onset, the efficiency of $\mathrm{Ca}^{2+}$ triggered exocytosis increases (Beutner and Moser 2001, Johnson, Marcotti et al. 2005). Two mechanisms are likely underlying this development in $\mathrm{Ca}^{2+}$ dependent exocytosis. First, it has been proposed that different sets of molecules are involved in driving exocytosis before and after the onset of hearing. For instance, IHCs may switches from an otoferlin-independent to an otoferlin-dependent mechanism during early postnatal days (Beurg, Michalski et al. 2010). Second, Cav1.3 channels undergo developmental refinement. It has recently been shown that there is an emergence of AZs with stronger synaptic $\mathrm{Ca}^{2+}$ influx and Cav1.3 cluster immunofluorescence, which was interpreted as an increase in the number of Cav1.3 channels, after the onset of hearing (Wong, Jing et al. 2013). Given the essential role of presynaptic $\mathrm{Ca}^{2+}$ in neurotransmitter release, reorganization of presynaptic Cav1.3 channels or a change in $\mathrm{Ca}^{2+}$ signaling may be accompanied with the change in firing properties. Interestingly, we indeed observed the emerging of ANFs with higher spontaneous rate (Figure 3.2) in mice around the onset of hearing, which coincide with the period when a larger Cav1.3 channel complement and stronger presynaptic $\mathrm{Ca}^{2+}$ signaling appear at a subset of synapses.

\subsubsection{Alternative mechanisms}

Evidences suggest that the ANF spontaneous discharge activity is controlled by presynaptic $\mathrm{Ca}^{2+}$ influx before and after hearing onset (Robertson and Paki 2002). It is unlikely that the spontaneous activity is generated by intrinsic activity of ANF, because manipulation cochlear perilymph with solution high in $\mathrm{Mg}^{2+}$ and low in $\mathrm{Ca}^{2+}$, which affects neurotransmitter release, led ANFs to fall silent (Siegel and Relkin 1987). Several other possible mechanisms exist to explain the emerging of high spontaneous rate fibers after the onset of hearing. First, the sensitivity of each fiber to glutamate release might increase after hearing onset. This may come about with postsynaptic GluA receptor upscaling or a change in intrinsic excitability, both of which may lead to change in the efficiency of action potential generation for a given similar level of glutamate input. However, in vivo and in vitro electrical physiology recordings suggest that ANFs have a very high sensitivity to presynaptic events, that nearly any synaptic event can generate an action potential (Russell and Sellick 1978, Siegel 1992, Rutherford, Chapochnikov et al. 2012). Therefore, this mechanisms might play a role in at least some fibers. 
Alternatively, the amount of neurotransmitter released spontaneously is controlled by the resting membrane potential, which can be regulated by several mechanisms. It has been shown that around the onset of hearing, the up-regulation of potassium conductance, voltage-activated $\mathrm{K}^{+}$channels of large conductance $(\mathrm{BK})$ enable a large hyperpolarizing conductance to maintain the resting membrane potential from generating action potential in response to transduction current. Thus it is unlikely that an increase in the resting membrane potential enables more neurotransmitter release at rest.

Another possibility is a change in endocochlear potential (EP) around onset of hearing. Pharmacological manipulation of EP by injection of furosemide was found to decrease both the EP and the spontaneous activity (Sewell 1984). Recordings showed the EP in mice reached mature level at around p11, increased during p12-16, and then recovered to its mature level again at p20 (Sadanaga and Morimitsu 1995). Therefore, the development of EP in infant mice may regulate the resting neurotransmitter release by affecting the driving force for the depolarization of membrane potential. However, no significant difference in SR was observed between p14-15 and p20-21 ANFs. Alternatively, a developmental change in ionic composition of endolymph, for example, potassium concentration, may affect the depolarizing electrical current in the HCs through mechanotransduction channels (Anniko and Wroblewski 1986).

\subsubsection{Emerging of ANF response heterogeneity}

After hearing onset, the transmission is controlled by graded membrane potential in IHC. One IHC connects to 10-30 afferent fibers, each with distinct firing properties in the absence of sound and stimulus dependency. Currently, it is hypothesized that single IHC decompose the auditory information contained in the membrane potential into functionally diverse ANFs via synaptic mechanisms (Frank, Khimich et al. 2009, Grant, Yi et al. 2010, Liberman, Wang et al. 2011). However, it is not entirely clear which mechanisms contribute most to this heterogeneity. Here, the result that the emergence of stronger presynaptic $\mathrm{Ca}^{2+}$ signal coincides with that of higher SR fibers supports presynaptic $\mathrm{Ca}^{2+}$ signaling heterogeneity as a likely candidate mechanism. For example, a variation in the number of channels would relate to ANF heterogeneity (Frank, Khimich et al. 2009). A computational model assuming difference in maximum presynaptic $\mathrm{Ca}^{2+}$ 
conductance is able to reproduce fibers with different SR as well as sensitivity to suprathreshold tone bursts (Sumner, Lopez-Poveda et al. 2002, Wong, Jing et al. 2013).

The heterogeneity of SR has been shown to correlate with fiber threshold and dynamic range in cat (Liberman 1978). One drawback of using mice to reproduce this observation is that it is difficult to record a large number of ANFs from a single animal. Between animal hearing threshold variation could mask this dependency. Despite the technique difficulties, Taberner and colleagues found indications of a weak correlation between spontaneous rate and fiber threshold (Taberner and Liberman 2005).

Certain presynaptic and postsynaptic morphological features have also been shown to be related to SR heterogeneity. Early morphological studies using electron and light microscopy combined with single unit labeling showed that ANFs with different spontaneous rate correlate with a number of morphological features. (1) ANFs innervate distinct location around IHCs with different presynaptic ribbon synapse complexity. Low spontaneous rate fibers connect with synapses from the modiolar face/basal pole of IHCs, which have larger ribbons and smaller receptor patches than synapses located in the opposite area of the cell (Liberman 1982, Liberman, Wang et al. 2011). (2) Low spontaneous rate fibers have also been shown to have a smaller postsynaptic bouton size and contain less mitochondria compared to that of high spontaneous fibers (Liberman 1980). (3) Low spontaneous rate fibers tend to have smaller fiber diameters (Liberman and Oliver 1984, Merchan-Perez and Liberman 1996). However, no direct experimental evidence is available to prove causality.

\subsubsection{Development of tuning properties}

Immature ANFs in mice showed higher tuning threshold and smaller tuning curve tip sensitivity (Figure 3.2). An improvement in threshold may result from the maturation of the external ear and middle ear, which perform much efficient transduction of acoustic stimuli to the inner ear. The broader tuning frequency selectivity in p14-15 mice is reminiscent of tuning curves from fibers after applying ototoxic drugs or acoustic trauma which damage OHCs, leading to reduced mechanical amplification. Similar observations in developing mice suggests during early onset of hearing, the OHCs mechanical amplification function does not yet reach mature level. Mechanical amplification of 
OHCs requires transduction currents. The EP difference between two and three weeksold mice cannot explain the immature active amplification. Although the light microscopic morphology of HCs and tectorial membrane have adult appearance and configuration by p8 (Mikaelian and Ruben 1965), hair bundles and transduction channels might not be fully developed at p14-15. Alternatively, the expression of motor proteins, prestin, may not reach adult levels to perform efficient amplification (Abe, Kakehata et al. 2007).

An elevation in fiber threshold can also be observed in rate-level functions, characterized by a shift to higher intensities. A pure lack of OHCs amplification will predict a loss of basilar membrane nonlinearity, which in turn, leads to steeper rate-level functions. In contrast to this prediction, single ANFs have similar slopes in p14-15 mice compared to that in p20-21. Similar observations were obtained when ototoxic drugs or acoustic trauma induced combined IHCs and OHCs damage (Heinz, Colburn et al. 2002, Heinz and Young 2004). This suggests that both OHC and IHC function did not reach mature levels in p14-15 mice.

\subsubsection{Development of responses to tone bursts}

In response to suprathreshold tone bursts at CF, ANFs in p14-15 and p20-21 mice showed typical temporal response patterns. The comparable adaptation time course indicated that stimulus driven neurotransmitter release kinetics reached mature level as early as p14, albeit with lower average onset and adapted discharge rates (Figure 3.3). In addition, the responses latency became shorter during maturation. Those single fiber properties in response to suprathreshold tone bursts were in general consistent with results obtained using indirect measurement, like auditory brainstem responses (ABRs), which reflect synchronous activity of ANFs. Both the latency and amplitude of ABR wave I undergo further developmental changes after hearing onset (Walsh, McGee et al. 1986, Walsh, McGee et al. 1986, Walsh, McGee et al. 1986). The shorter latency and larger wave I amplitude of ABR wave I in p20-21 mice are consistent with direct measurements from single fiber recordings.

Single fiber recordings revealed a decrease in response latency of $1.5 \mathrm{~ms}$ during maturation from p14-15 to p20-21. The longer latency in p14-15 might be due to 
immature middle ear conduction, synaptic delay, spiral ganglion neuron spike generation or conducting velocity. Middle ear conduction deficits have been found to lead to ABR latency increases. However, the latency increases might be due to the attenuation of sound intensity arriving in the cochlea. Therefore, the immature conduction by external and middle ear could introduce a delay in the response. Basilar membrane properties are also not likely to introduce a delay long enough to account for single fiber latency, given the length of basilar membrane and conduction velocity of mechanical vibration in the cochlear fluid. A synaptic delay, spike generation and spiral ganglion conduction velocity may account for most part of the delay in p14-15 ANFs. An analysis of ABR wave I latencies in kitten suggested nerve conduction time during first postnatal week can account for $0.3 \mathrm{~ms}$ total latency delay, leaving a synaptic delay of about $1.4 \mathrm{~ms}$. Another possible source of the improvement in response latencies with maturation may be due to the emerging of higher fraction of large, "monophasic" EPSC waveforms (Grant, Yi et al. 2010). A difference in EPSC waveforms has been suggested to be able to shape the spike latency (Rutherford, Chapochnikov et al. 2012). It is also possible that during maturation, an increase in fiber diameters and myelination shortens the single fiber response latencies in p20-21 ANFs.

The smaller average onset and adapted discharge rate in p14-15 ANFs is most likely a result of events downstream of the endocochlear potential (EP) because EP reach mature level at around p11 in mice (Sadanaga and Morimitsu 1995). For example, p14-15 IHCs might have less transduction channels on its hair bundles, or IHCs may have smaller readily releasable pool size and slower neurotransmitter replenishment rate. 


\subsection{Bassoon and synaptic ribbon in sound encoding}

IHCs use graded receptor potential to transmit continuously time-varying signals. The IHC ribbon synapses are remarkable in their capability to sustain high rate of transmission for relatively long period of time and still preserve submillisecond precision of sound encoding (Geisler 1998). How the ribbon synapses support such function is still not well understood. In this study, we took advantage of two available mouse lines with genetic manipulations that disrupt presynaptic scaffold protein Bassoon to different extent to better differentiate ribbon-dependent effects. We propose that leaky expression of Bassoon in IHCs of $\mathrm{Bsn}^{\mathrm{gt}}$ mice supported better but still loose anchorage of synaptic ribbons. Bsn ${ }^{\mathrm{gt}}$ IHCs ribbon-occupied AZs had better preserved $\mathrm{Ca}^{2+}$ channel clusters compared to ribbonless AZs. Regarding IHC cell physiology, Bsn ${ }^{\text {gt }}$ IHCs showed an intermediate reduction of peak $\mathrm{Ca}^{2+}$ currents and sustained exocytosis compared to that of $\mathrm{Bsn}^{\Delta \mathrm{Ex} 4 / 5} \mathrm{IHCs}$. However, IHC fast exocytosis as well as single ANF responses showed almost identical response properties in both mutants. If the ribbon-occupied synapses of mature $\mathrm{Bsn}^{\mathrm{gt}}(44 \%)$ or $\mathrm{Bsn}^{\Delta \mathrm{Ex} 4 / 5}(12 \%)$ mice were fully functional, we would expect a subpopulation of ANFs with WT-like firing responses in both mutants and on average better responses in Bsn ${ }^{\mathrm{gt}}$ ANF. However, neither expectation was fulfilled. We conclude that these remaining anchored ribbons in Bsn mutant IHCs are functionally inadequate due to looser anchorage, or there was a ribbon-independent effect of the bassoon disruption on synaptic function.

\subsubsection{Ribbon-occupied vs. ribbonless synapses}

At least two functions of synaptic ribbon have been suggested. First, the ribbon promotes large complements of synaptic vesicles and $\mathrm{Ca}^{2+}$ channels to maintain a sizable readily releasable pool. Second, the ribbon might accelerate the refilling of vesicles after depletion of the fast component of exocytosis. The differential level of ribbon loss in $\mathrm{Bsn}^{\Delta \mathrm{Ex} 4 / 5}$ and $\mathrm{Bsn}^{\mathrm{gt}}$ allowed us to explicitly study the effect of ribbon loss on synaptic phenotypes.

The deficit of ribbon anchorage in $\mathrm{Bsn}^{\Delta \mathrm{Ex} 4 / 5}$ was firstly reported by Dick et al. (2003) in photoreceptor ribbon synapses. They demonstrated the absence of attached ribbons 
throughout postnatal retinal development and argued for a role of Bassoon in synaptogenesis. This is different from what we observed in IHCs. In $\mathrm{Bsn}^{\Delta \mathrm{Ex} 4 / 5} \mathrm{IHCs}$, there was a gradual decrease of ribbon-occupied synapses from $47 \%$ at onset of hearing to $22 \%$ at 3 weeks of age (Frank, Rutherford et al. 2010) to $12 \%$ at 10 weeks of age (Khimich, Nouvian et al. 2005). It is more likely that in $\mathrm{Bsn}^{\Delta \mathrm{Ex} 4 / 5} \mathrm{IHCs}$, ribbons are initially present at most active zones, but cannot be stabilized in the absence of Bassoon.

$\mathrm{Bsn}^{\mathrm{gt}} \mathrm{IHCs}$ maintained a higher fraction of ribbons (44\%) throughout development. No Bassoon protein was detected in the brain of $\mathrm{Bsn}^{\mathrm{gt}}$ mice by western blot (Hallermann, Fejtova et al. 2010). Our results of immunostaining showed weak bassoon immunofluorescence at a few Bsn ${ }^{\text {gt }}$ synapses (Figure 3.7) and $3 \%$ bassoon mRNA in realtime PCR experiments revealed a weak leaky expression of Bassoon, which might support the long term anchorage of a fraction of ribbons. Alternatively, the $180 \mathrm{kD}$ protein fragment expressed in $\mathrm{Bsn}^{\Delta \mathrm{Ex} 4 / 5}$ may interfere with long term stabilization of synaptic ribbons by interfering protein interactions at AZs. This possibility is less plausible for two reasons. First, the fragment does not localize to synapses (Altrock, tom Dieck et al. 2003, Frank, Rutherford et al. 2010). Second, a dominant negative effect of the fragment is unlikely because $\mathrm{Bsn}^{\Delta \mathrm{Ex} 4 / 5}$ heterozygous mice had normal IHC exocytosis and ANF spike discharge rates as well as ABRs.

Despite the difference in the fraction of ribbon-occupied synapses, ribbon-occupied synapses of both Bsn mutants showed abnormal but stronger Cav1.3 immunofluorescence than ribbonless synapses (Figure 3.8) (Frank, Rutherford et al. 2010). Functionally, Bsn ${ }^{\text {gt }}$ IHCs had lesser reduction of whole-cell $\mathrm{Ca}^{2+}$ current than in $\mathrm{Bsn}^{\Delta \mathrm{Ex} 4 / 5} \mathrm{IHCs}$. Ribbonoccupied synapses in both $\mathrm{Bsn}^{\Delta \mathrm{Ex} 4 / 5}$ and $\mathrm{Bsn}^{\mathrm{gt}} \mathrm{IHCs}$ also tend to have more membrane proximate vesicles compared to that of ribbonless synapses, but still much smaller compared to WT synapses (Frank, Rutherford et al. 2010). These results are consistent with a role of synaptic ribbon in stabilizing presynaptic Cav1.3 channels and readily releasable pool of vesicles. However, ribbons, due to the looser anchorage, failed to exert the full power. As a consequence, both mutants showed similar fast exocytosis in vitro and evoked spike rates when assessing the sound encoding at individual synapse level by single unit recording from ANFs. 


\subsubsection{Discrepancy between cell physiology and single unit results}

In vitro physiology data showed intermediate phenotypes in $\mathrm{Bsn}^{\mathrm{gt}} \mathrm{IHCs}$ in aspects of $\mathrm{Ca}^{2+}$ current, sustained exocytosis and vesicle replenishment, but not sound encoding in vivo. This discrepancy is partly due to the experimental approaches. The in vitro cell physiology experiments assess the behavior of a whole IHC, while single fiber recording samples responses from the output of an individual synapse. The better preserved whole cell $\mathrm{Ca}^{2+}$ current in $\mathrm{Bsn}^{\text {gt }}$ compared to that in $\mathrm{Bsn}^{\Delta \mathrm{Ex} 4 / 5}$ IHCs does not necessarily mean that there were more Cav1.3 channels at individual synapse to increase the number of docked vesicles. There could be more extra-synaptic $\mathrm{Ca}^{2+}$ channels in $\mathrm{Bsn}^{\mathrm{gt}} \mathrm{IHCs}$. Indeed, there was a tendency for a greater proportion of extrasynaptic Cav1.3 immunofluorescence in IHCs lacking bassoon in both mutants (Frank et al). Although the number of membrane-proximal vesicles of ribbon-occupied synapses tended to be slightly larger than that of ribbonless synapses in IHCs of both mutants, their number did not reach WT levels (Frank, Rutherford et al. 2010).

Better vesicle replenishment in $\mathrm{Bsn}^{\mathrm{gt}}$ mutant was only observed in vitro but not in vivo. This could be partly explained by different experimental conditions. The recovery time constant in vitro is about $140 \mathrm{~ms}$ for the fast and largest component of recovery (Moser and Beutner 2000), which is much longer than that observed in vivo (47 ms, Figure 3.13). Different temperatures between these two experiments may play a major role in such discrepancy (in vitro: room temperature vs. in vivo: $35^{\circ} \mathrm{C}$ ). The age of the mice used for the two experiments also differ (in vitro: $3-4$ weeks vs. in vivo: 5-11 weeks). However, this unlikely made a major contribution because preliminary data showed no indication of age-dependent changes in RRP recovery time course (data not shown). In addition, the IHC membrane potential was held at $-87 \mathrm{mV}$ during the in vitro experiment. In contrast, the receptor potential in vivo may be more depolarized and may lead to calciumdependent enhancement of vesicle replenishment to the synapses. Thus, the experimental conditions in vitro might have favored the detection of subtle differences 


\subsubsection{Direct consequences of Bassoon disruption on active zone function or effect of looser ribbon anchorage?}

At the individual synapse level, the mere presence or absence of a synaptic ribbon seems not to improve the sound encoding in ANFs. This can be explained by (1) disruption of Bassoon causes ribbon-independent perturbations of the active zone organization and interfere with the synaptic transmission which may occlude a possible ribbon effect. (2) The remaining ribbons are functionally inadequate to support normal function.

Bassoon is thought to not be directly involved vesicle exocytosis but rather to play a role in synaptic vesicle clustering in conventional synapses of cultured hippocampus neurons (Mukherjee, Yang et al. 2010) and vesicle replenishment in cerebellar mossy fiber to granule cell synapses (Hallermann, Fejtova et al. 2010). In IHCs, a bassoon disruption causes a reduction of synaptic $\mathrm{Ca}^{2+}$ channels, membrane proximal vesicles and their replenishment, while the unaltered EGTA sensitivity of exocytosis in Bsn mutant IHCs suggested that the coupling of $\mathrm{Ca}^{2+}$ influx and exocytosis is not strongly effected (Figure 3.9C). Moreover, $\mathrm{Ca}^{2+}$ uncaging revealed normal exocytic responses arguing against a negative effect on $\mathrm{Ca}^{2+}$ dependent fusion. The quest of how much of this is due to direct effects of bassoon deficiency or to the absence of the ribbon triggered this study. However, we cannot provide a definitive answer, because the remaining ribbons in BGT inner hair cells may not be fully functional. 3D imaging analysis of the center of mass distance between ribbons and Cav1.3 or GluA2 receptor clusters revealed an enlarged distance in mutant ribbon-occupied synapses. This result provides quantitative support to the notion of a looser anchorage at ribbon-occupied synapses in BSN ${ }^{\mathrm{gt}}$ IHCs. It is less unlikely that the larger distance would reflect an increase size of synaptic ribbons or the presence of multiple ribbons at many synapses in $\mathrm{BSN}^{\mathrm{gt}}$ IHCs, as RIBEYE immunofluorescence intensity for ribbon-occupied synapses in $\mathrm{BSN}^{\mathrm{gt}}$ was not increased compared to that in wild-type IHCs.

Given the fact that Bassoon locates at the base of photoreceptor ribbon synapses and a synaptic ribbon anchorage deficit in Bsn mutants, it is more likely that Bassoon plays role in ribbon anchorage. IHC ribbon synapses are tethered to presynaptic membrane by a single arcuate density structure (Sobkowicz, Rose et al. 1982). The molecular composition of this arcuate density is unknown, but Bassoon is a potential candidate. The 
direct physical interaction between Bassoon and RIBEYE makes it possible to perform such function (tom Dieck, Altrock et al. 2005). Therefore, we favor the hypothesis that upon genetic disruption of Basson, the anchorage of ribbons is looser at the ribbonoccupied synapses of $\mathrm{BSN}^{\mathrm{gt}} \mathrm{IHCs}$. An increased distance to the AZ could impair a putative function of the ribbon in promoting a large number of $\mathrm{Ca}^{2+}$ channels and docking sites at the AZ and facilitating vesicle replenishment. This could come about due to the reduced effect of ribbon-associated proteins with functions in scaffolding or regulation of docking and priming.

Future experiments are needed to better distinguish the scenarios. Emerging methods like acute photoablation of synaptic ribbons (Snellman, Mehta et al. 2011) may help reveal the role of remaining ribbons in $\mathrm{BSN}^{\mathrm{gt}} \mathrm{IHCs}$. However, the small average number of ribbon-occupied synapses, and low RRP size make this experiment very challenging. Postsynaptic bouton imaging/patch could also be used for this purpose. But it is difficult to apply this techniques on old mice after p14. The availability of more genetically modified animals (Sheets, Trapani et al. 2011) made it possible to test function(s) of ribbon-associated proteins. All those various approaches ultimately may help revealing the functional roles of synaptic ribbons $\mathrm{BSN}^{\mathrm{gt}} \mathrm{IHCs}$.

\subsubsection{Alternative possible functions of Bassoon in ribbon synapses and conventional synapses}

How does the disruption of Bassoon leads to disorganized active zone in both ribbon types and conventional synapses is still unclear. The large molecular size of Bassoon has hindered the study of its biochemical properties. However, studies of active zone organization in normal and pathological conditions have provided us several potential pathways underlying the abnormal phenotype. At conventional synapses, Bassoon might have a role in normal synaptogenesis (Lanore, Blanchet et al. 2010) because recruitment of Bassoon is one early step in the development of synapses (Zhai, Olias et al. 2000). During early synaptogenesis, Bassoon, Piccolo and RIM containing transport vesicles function as preassembled $\mathrm{AZ}$ precursor vesicles, carrying a set of $\mathrm{AZ}$ proteins to initialize further development of AZs (Shapira, Zhai et al. 2003) and postsynaptic differentiation (Friedman, Bresler et al. 2000). Precursors have also been reported in the assembly of 
photoreceptor ribbon synapse (Regus-Leidig, Tom Dieck et al. 2009). The transportation of several cytomatrix proteins Bassoon, Piccolo, RIBEYE and RIM1 in precursor spheres to the presynaptic sites precedes the appearance of other presynaptic proteins like Munc13, CAST1, RIM2 and L-type $\mathrm{Ca}^{2+}$ channels alpha1 subunits (Regus-Leidig, Tom Dieck et al. 2009). The interaction of Bassoon with other cytomatrix proteins and early appearance at presynaptic AZs suggest that Bassoon is involved in early steps of synaptogenesis. Indeed, Bassoon mutation in photoreceptor leads to delayed transport of precursor spheres and degradation of RIBEYE and Piccolo protein levels (Regus-Leidig, tom Dieck et al. 2010). Recently, Bassoon has been shown to be able to interact with E3 ubiquitin ligase Siah1, through which involved in regulating presynaptic proteostasis (Waites, Leal-Ortiz et al. 2013). In inner hair cells lacking functional Bassoon $\left(\mathrm{Bsn}^{\Delta \mathrm{Ex} 4 / 5}\right)$, an accumulation of tubular and cisternal structures has been observed near the synapses (Khimich, Nouvian et al. 2005). An interesting hypothesis is that the accumulation of these tubular and cisternal structures may indicate the presence of abnormal presynaptic protein recycling dynamics.

\subsubsection{ANF sound coding deficit in Bsn mutants}

A directly consequence of disorganized AZs by bassoon disruption in IHC ribbon synapses is the reduction of spontaneous and evoked firing rates. The presence of synapse-anchored ribbons in $\mathrm{Bsn}^{\mathrm{gt}}$ mutant IHCs improved $\mathrm{Ca}^{2+}$ channel clustering and sustained exocytosis. However, the readily releasable pool size was equally disrupted regardless of the presence or absence of synaptic ribbon, and release machinery was not affected in the absence of functional bassoon and synaptic ribbon in mutant IHCs. Computational models assuming a readily releasable pool to hold certain amount of neurotransmitters, which is released and replenishment by diffusion process can reproduce the reduction in vesicle fusion as well as account the reduction of firing rate in vivo (Buran, Strenzke et al. 2010, Frank, Rutherford et al. 2010). Although so far only the mean response across ANFs has been modeled, it faithfully reproduce the behavior of fibers to suprathreshold tone bursts in Bsn mutants.

It has been shown that $\mathrm{OHC}$ and IHC lesions can produce different changes in the discharge behavior of ANFs to sound stimuli. Damage of OHCs lead to an absence of 
compressive nonlinearity. The function of basilar membrane vibration and input sound pressure levels will be shifted to higher SPLs and become linear. As a consequence, the slope of rate-level function will be steeper, and the tuning curve tip will be broadened. This is not observed in Bsn mutation. Normal DPOAE (Khimich, Nouvian et al. 2005) confirmed the presence of normal mechanical amplification. Thus, the phenotype in ANFs firing rate is mainly a result of IHC lesions. One interesting finding is that reduced firing rate and shallow rate-level function can also be observed if IHCs are damaged by acoustic trauma (Liberman and Dodds 1984) or ototoxic drugs, which compromises cochlear transduction and lower the firing rate of ANFs, more specifically, the disruption of presynaptic neurotransmission. Lesions in IHCs induced by ototoxic drugs can produce a shallower rate-level function, similar to the observations we made in single fiber recordings.

The sound encoding phenotypes might also reflect a change in efferent connection of IHC-SGN synaptic complex. Lateral olivorcochlear efferent (LOC) fibers originating from later superior olivary nucleus (LSO) neurons form Bassoon-containing synapses with the postsynaptic boutons of SGNs below IHCs (Liberman, Dodds et al. 1990). It remains unclear if efferent synaptic transmission is abnormal in Bsn mutant mice, although we did not have indication of the contribution of efferent connections. The functional role of efferent connections is not yet entirely clear: they probably modulate the functional state of the postsynaptic bouton of SGN. In cat ANFs, the spontaneous rate can be partly regulated by the efferent innervation (Guinan and Gifford 1988). It is possible that part of the reduction in firing rate is from reduced efferent input. Further experiments are needed to clarify the efferent function. 


\subsection{Auditory nerve fiber responses in BLSW mice}

\subsubsection{Sound encoding phenotypes in BLSW cochlea}

The normal hearing process requires proper function of a series of peripheral mechanisms eventually leading to sound encoding. Consequently, mutations affecting any stage between hair cells and SNGs, as well as a disruption of general cochlear morphology and function, may potentially lead to SNHL. This is one reason why the genetic basis underlying SNHL shows large heterogeneity. The present study shows that ANFs of BLSW mice had abnormal tuning threshold and sharpness of tuning, which is consistent with the previous finding of OHC dysfunction. Detailed analysis showed BLSW ANFs have elevated spontaneous discharge activity compared with controls. At the level of individual synapses, the IHC-afferent transmission was largely unaffected. P14-18 ANFs of BLSW mice even showed significantly larger onset responses and a tendency for shorter response latencies albeit the $\sim 60 \%$ reduction in maximum IHC transduction currents (Charizopoulou, Lelli et al. 2011). Those observations are consistent with the hypothesis that the reduced maximum IHC transduction currents lead to greater depolarization due to lack of large conductance $\mathrm{K}^{+}$current, therefore, compensate the smaller transduction currents. However, a lack of functional large conductance voltageand $\mathrm{Ca}^{2+}$ activated $\mathrm{K}^{+}$channels $\left(\mathrm{BK}_{\mathrm{Ca}}\right)$ has been shown to degrade precision of spike timing (Oliver, Taberner et al. 2006). The preserved spike timing of BLSW ANFs indicates that there might be other compensatory changes in IHCs, for example, upregulation of $\mathrm{Ca}^{2+}$ channels, which maintain the membrane time constant and precision of spike timing. Indeed, cell physiology experiments indicated that BLSW IHCs have larger $\mathrm{Ca}^{2+}$ peak current, larger RRP and sustained exocytosis compared with control IHCs.

The ANF sound encoding phenotypes confirmed the age-related hearing loss (ARHL) phenotype in BLSW mice. Beside BLSW mice, other inbred mouse strains also exhibit ARHL, with distinct onset time and pathological changes. For example, C57BL/6J mice exhibit significant hearing loss for high frequencies already at 6 months, and low frequency hearing loss at 12 months. CBA/J mice show minimal hearing loss by 18 months. The elevated ABR threshold is usually accompanied with degeneration of the 
organ of Corti or ANFs. Genetic linkage analysis identified heterogeneity in the locus underlying ARHL in different mouse strains. It has been identified that the gene encoding cadherin 23 (Cdh23) is underlying the SNHL in C57BL/6J mice. Cdh23 has been shown to be one component of the tip link of stereocilia, and mutations of $\mathrm{Cdh} 23$ lead to hair cell deficits. BLSW mice exhibit hearing loss much earlier than C57BL/6 mice, which were used as controls for this study.

\subsubsection{Age dependent ABR threshold changes}

Previous ABR data show a high wave I amplitude at 2 weeks of age. However, the mean ABR wave I amplitude of BLSW mice was comparable to that of C57BL/6 mice at 2 weeks of age in our study. At 3 weeks of age, the amplitude of BLSW mice is significantly smaller than that at 2 weeks of age. Immunostaining did not show signs of synapse loss between 2 and 3 weeks of age. Single fiber recordings revealed a threshold elevation between 2 and 3 weeks of age but similar maximum discharge rates in response to suprathreshold stimuli. Those results lead us to the hypothesis that a smaller number of ANFs is recruited given the same level of sound stimuli, therefore leading to a smaller ABR wave I amplitude of 3 week-old compared to that of 2 week-old BLSW mice

\subsubsection{ANF sound encoding and hair cell transduction}

It has previously been shown that BLSW IHCs and OHCs lack large conductance $\mathrm{K}^{+}$ currents at p12. Consequently, a smaller transduction current can lead to a larger depolarization. This would also predict an elevated resting membrane potential. The observation that BLSW ANFs have higher mean spontaneous discharge rates compared to control mice is consistent with this prediction. However, we cannot exclude the possibility that there is a population of low spontaneous rate fibers, which fail to be detected using 90-100 dB noise bursts due to the elevated hearing single fiber threshold. This would lead to an overestimation of mean spontaneous discharge rate in BLSW ANFs. On the other hand, the smaller maximum hair cell transduction currents lead to a much smaller range of sound intensities that the hair cell can respond to, which can also cause 
steeper rate-level functions. However, the reduced transduction currents in BLSW IHCs may still depolarize membrane potential to a level comparable to that in normal IHCs. At p14, BLSW ANFs discharged with a significantly larger onset rate compared to that of control ANFs, which is consistent with the prediction of larger depolarization. Our in vitro studies suggested that BLSW IHCs have larger maximum $\mathrm{Ca}^{2+}$ currents, larger size of RRP and sustained exocytosis. Larger maximum $\mathrm{Ca}^{2+}$ currents might be due to more $\mathrm{Ca}^{2+}$ channels. It is possible that the enlarged RRP may be related to a homeostatic change in IHCs given smaller transduction current and altered membrane potential.

Furthermore, BLSW ANFs can still encode amplitude modulation tones. The ability of IHC membrane potential in encoding amplitude modulation is limited by the membrane time constant. Normal IHCs exhibit large rapidly activating potassium currents $\left(\mathrm{I}_{\mathrm{k}, \mathrm{f}}\right)$, which lower the membrane time constant to below $1 \mathrm{~ms}$. The over $50 \%$ reduction in potassium current in BLSW IHCs would lead to a higher membrane time constant, therefore, a degraded ability to encode high frequency amplitude modulation. Indeed, BLSW ANFs showed a tendency of degraded ability to encode amplitude modulation at a frequency of $1 \mathrm{kHz}$ without reaching significant (Figure 5.7). $\mathrm{I}_{\mathrm{k}, \mathrm{f}}$ has been shown to be abolished in the absence of BK channels (Oliver, Taberner et al. 2006). Different from BK channel knockout mice with nearly a complete absence of fast component of potassium currents, BLSW IHCs have both fast and slow component, albeit with a much reduced amplitude. Interestingly, the BK channels knockout IHCs have similar voltage responses to current injection compared to BLSW IHCs. However, the ANF spike rates were smaller in BK channels knockout in comparison with controls. The larger depolarization of membrane potential did not lead to higher spike rate in vivo.

\subsubsection{Mechanisms underlying GIPC3 mutation on cochlear functions}

The molecular pathways that GIPC3 affecting the SNHL in BLSW mice remain unknown. The PDZ domain of GIPC3 shares $71 \%$ and $66 \%$ sequence identity to GIPC1 and GIPC2, respectively. The capability of GIPC protein family to bind multiple receptors in various cellular signaling pathway suggests that GIPC3 could act as an adaptor protein. Currently, little is known about the interacting partner of GIPC protein in vivo. However, GIPC protein can interact with GAIP, a membrane-anchored GTPase activating protein for Gai3 
subunits, which locate to clathrin-coated vesicles in the Golgi region. Those results implicate that GIPC may participate in sorting proteins for vesicular trafficking. Here, possible pathways that GIPC3 which might participate in given the available experimental data will be discussed.

\subsubsection{Hair bundle development}

Using mouse mutants have helped identifying important molecules involved in sound transduction. This process requires proper structure and function of stereocilia. Currently, several classes of molecules have been identified underlying the development and function of the stereocilia bundles. One of the important molecule classes is myosins in stereocilia. Mutation in different myosins lead to hearing as well as vestibular deficit. In the spontaneously arisen mouse mutant shaker 2, mutations in myosin 15 lead to abnormally short and irregular stereocilia bundle morphology (Probst, Fridell et al. 1998). In another study, the mouse shaker-1 locus, which encodes myosin VIIA, was found to be required for hair cell stereocilia bundle organization and has a role in the function of cochlear hair cells (Self, Mahony et al. 1998). Those studies have revealed a complex protein network involved in maintaining structure and function of stereocilia bundle functions. BLSW mice show disoriented OHC bundles, less rigid and sparse IHC bundles already at postnatal day 3 . Abnormal $\mathrm{OHC}$ bundles are expected to lead to a mechanical amplification deficit. This was indeed observed in single fiber responses, which showed elevated thresholds, loss of sharp tuning and steep rate-level functions. Similar single fiber property changes can also be observed in noise traumatized animals or after tha application of ototoxic drugs. Therefore, it is likely that in BLSW mice, a mutation in GIPC3 leads to bundle maturation deficits due to impaired transporting of key molecules important for the maintenance of hair bundle morphology and function.

One important class of protein involved in vesicular trafficking are Myosins, which function as molecular motors. They produce movement alone the actin filament by interacting with F-actin microfilaments using a conserved domain and hydrolyzing ATP. Different classes of Myosins have distinct C-terminals. It has been hypothesized that the unique C-terminus targets different Mysoin to distinct cargo and subcellular regions. The similarity of GIPC3 PDZ domain with GIPC1 implicate that both protein may share similar binding partners. Different C-terminal of GIPC1 and GIPC3 may bind to different 
types of Myosins to target cargo proteins to different cellular locations. It has been shown that GIPC1 can interact with Myosin VI and the myosin VI-GIPC1 protein complex. GIPC1 can regulate stereociliary bundles and cell polarization by involving in the trafficking of Van Gogh-like 2 (Vang12), which can mediate planar cell polarity signaling (Giese, Ezan et al. 2012). Both reduction and overexpression of GIPC1 in HCs lead to disrupted hair bundle morphology.

\subsubsection{Potassium channel membrane trafficking}

Another role of GIPC3 might be the trafficking of potassium channels to the membrane. BLSW IHCs exhibit normal potassium currents at postnatal day 6. However, at p12/13, both the amplitude of potassium current is much reduced compared with that of controls (Charizopoulou, Lelli et al. 2011). Both fast component and slow component are still present. Therefore, the reduced potassium currents might be due to a reduced number of potassium channels, for example BK channels, on the IHC membrane. A direct interaction of BK channels with GIPC3 has not bben described. Further biochemical and morphological studies are needed to verify possible role of GIPC3 in BK channel trafficking.

\subsubsection{Exocytosis}

Within IHCs GIPC3 is distributed similar to Vglut3 and Myosin VI (Charizopoulou, Lelli et al. 2011), both of which are involved in synaptic transmission. The location of Myosin VI near active zones overlaps with tubular structures that have been considered to be endocytotic structure, suggesting a role of Myosin VI in synaptic vesicle recycling (Heidrych, Zimmermann et al. 2009, Roux, Hosie et al. 2009). Our capacitance measurements suggested larger Cav1.3 currents yet similar exocytosis efficacy. The neurotransmission is minimally disturbed as single ANF responses have comparable maximum discharge rates and and adaptation time courses. It is possible that the remaining small amount of GIPC3 protein in BLSW IHCs is enough for performing efficient vesicle recycling. However, when challenged with continuous stimulation, the GIPC3 dependent protein/vesicle recycling might suffice to catch up with the neurotransmitter release rate, and lead to a slower sustained neurotransmitter release during long stimuli. Further work is needed to test this possibility. 
GIPC3 is not only expressed at IHCs but also in spiral ganglion neurons (Charizopoulou, Lelli et al. 2011). No experimental data is currently available on the role of GIPC3 on spiral ganglion neuron function. However, the BLSW exhibit autogenic seizures, suggesting a more general disruption of higher brain function in this mouse line. The mechanism of susceptibility is not well understood. Given the fact that the PDZ domain of GIPC3 can potentially interact with wide range of different proteins, reduction of GIPC3 protein level or loss of function might interfere with multiple cellular pathways. Identifying the interaction partner of GIPC3 may ultimately help reveal important molecules for $\mathrm{HC}$ transduction. 


\subsection{Optogenetic stimulation of mouse cochlea}

In the last part of the thesis, light-evoked responses of single ANF/cochlear nucleus neuron were characterized in ChR2 transgenic mice and mice expressing the ChR2variant CatCh. This is the first study to demonstrate the utilization of optogenetics tools in the cochlea. More specifically, this study focused on single unit response properties upon optogenetic stimulation. Neurons in the region of auditory nerve, cochlear nucleus as well as inferior colliculus fire optically driven spikes with different latencies and precisions. To achieve specific stimulation of ANFs, ChR2-variant CatCh was expressed in SGNs by virus-mediated transduction. The activation of auditory neurons was confirmed in CatCh mice by demonstrating acoustically evoked responses of light responsive neurons. In comparison with $\mathrm{ChR} 2$ transgenic mice, neurons in CatCh mice showed optically evoked spikes with shorter latencies and better temporal precison.In addition, neurons in CatCh mice required less laser energy to achieve excitation. Those results are consistent with the previously reported ultra light-sensitive and fast neuronal excitation properties of CatCh (Kleinlogel, Feldbauer et al. 2011).

\subsubsection{Optogenetic stimulation of auditory neurons}

Two different methods were used to achieve ChR2 expression in ANFs. In transgenic mice, ChR2 expression was driven by Thy 1.2 promoter. Selective expression of ChR 2 in different cells of the mouse nervous system has been reported (Arenkiel, Peca et al. 2007). The ChR2 was found to be expressed throughout the spiral ganglion, the cochlear nucleus and MNTB, but not in hair cells and supporting cells of the organ of Corti by immunohistochemistry. Within the cochlea, $\mathrm{ChR} 2$ was restricted to the somata, peripheral as well as central axons of most afferent neurons. Therefore, optogenetic activation of afferent neurons can be attributed to afferent neurons but not hair cell stimulation. It is not clear if spikes were generated on somata or peripheral neurites of afferent neurons. Further patch clamp analysis of response properties of afferent neurons upon optogenetic stimulus was hindered by late expression of ChR2 (after $3^{\text {rd }}$ postnatal weeks) and challenging experiment to record from mature afferent neurons. AAV6-mediated expression of $\mathrm{CatCh}$ resulted in restricted expression of $\mathrm{CatCh}$ in spiral ganglion neurons. Unlike the transgenic ChR2 expression, there was a strong basoapical gradient for AAV6- 
mediated CatCh expression. Therefore, a laser fiber was inserted into the round window to stimulate afferent neurons in the basal part of the organ of Corti.

Robust single unit activity could be evoked by illumination with blue light. It cannot be excluded that in transgenic $\mathrm{ChR} 2$ mice, neurons other than afferent nerves being excited by light. However, the observation of neurons in the region of ANFs, cochlear nucleus and inferior colliculus confirmed the activation of auditory pathway. Similar optically evoked spiking patterns were observed from neurons in CatCh mice. The response of light-sensitive neurons to acoustic stimuli confirmed the activation of auditory neurons. Mapping the frequency selectivity of the neurons indicated CFs around 10-16 kHz, which is the middle turn of organ of Corti. Considering the basoapical gradient of CatCh expression in the organ of Corti, the expression level at $10-16 \mathrm{kHz}$ region might be sufficient for activation.

Another method for optical stimulation of ANFs is to use infrared irradiation (Littlefield, Vujanovic et al. 2010). Different from the transcochlear stimulation by cochleostomy and round window insertion, they placed a laser fiber against round window membrane without insertion. Successful stimulation of ANFs have been achieved by orienting the laser fiber towards SGNs. Possible mechanisms by infrared laser excitation including a transient increase in tissue temperature via energy absorption by water. The response latency in their study is $2.5 \mathrm{~ms}$ to $4.0 \mathrm{~ms}$, which is in the similar range of latencies of ANF spiking evoked by optogenetic stimulation. A direct interaction of $473 \mathrm{~nm}$ laser light with the tissue seems to be unlikely because no optically evoked potentials could be recorded in animals without $\mathrm{ChR} 2$ expression. Although the stimulation rate in both cases degraded at $100 \mathrm{~Hz}$, small pulse rate may be sufficient for transmitting information for speech. Further studies are needed to establish the maximum rate at which ANFs can follow optogenetic stimulation.

\subsubsection{Outlooks}

This study provide new possibility to use optogenetics in auditory research and potentially an alternative strategy for cochlear stimulation. The activation of auditory neurons by blue light was first characterized. However, several questions need to be addressed for the application of this technique. First, how and where are light-evoked spikes generated 
in the ANFs? Because ChR2 is expressed on both the neurites and cell body, illumination of the whole neuron may activate multiple sites. It is possible that one of the node of Ranvier where the highest ChR2 is expressed will dominate the pattern of evoked spikes. Further modeling works and in vitro experiments are needed to address this question. Another question is how the neuronal sensitivity to light related to the expression level of ChR2 in SGNs. The activation of auditory neurons of CF about 10-16 kHz (Figure 6.3) and basoapical gradient of CatCh expression along the organ of Corti suggested that relatively low amount of ChR2 might be sufficient for optical stimulation. Future works integrating morphological and physiological studies are needed to advance this emerging field. Towards a clinical translation of optogenetic CIs, a safe method for transgenic ChR2 expression in human SGNs and the development of multichannel optical stimulation technologies are still necessary. 


\section{Summary}

Afferent synapses between inner hair cells (IHCs) and spiral ganglion neurons in the cochlea transfer sound information into a discrete spikes code. The spiking responses provide us a window to directly observe the output of the cochlea. The availability of mutant strains with genetic hearing impairment make mouse a valuable species to investigate the molecular mechanisms of sound encoding in the cochlea. The main focus of this work is to use the mouse as a model species to study the cochlear sound encoding by recording single unit activities from auditory nerve fibers (ANFs) in vivo.

First, developmental changes of ANF responses before and after hearing onset were characterized as an introduction on how normal ANF responses mature during the early postnatal age. Mouse inner ears undergo a series of developmental changes, but previously nothing was known about single fiber response properties. In this chapter, the response properties of ANFs before (p10-11) and after hearing onset (p14-15, p20-21) were characterized for the first time. It was found that mice ANF responses during early postnatal development share many properties observed in other mammalian species. Spontaneous bursting activity from ANFs/cochlear nucleus neurons was observed before hearing onset. The concurrent emergence of high spontaneous rate ANFs around hearing onset and stronger presynaptic $\mathrm{Ca}^{2+}$ signaling and larger $\mathrm{Cav} 1.3$ channel complement was first demonstrated. This observation leads to a hypothesis that presynaptic mechanisms and, more specifically, $\mathrm{Ca}^{2+}$ channel organization and signaling, contribute to the establishment of heterogeneity in the population of ANFs. After hearing onset, single fiber responses gradually reach maturation from p14-15 to p20-21. The improvement of tuning properties and frequency selectivity suggested an immature functioning of OHCs in p14-15 mice. Rate-level functions indicated a possible immature IHCs function at p1415, which was consistent with longer response latencies and smaller maximum evoked discharge rate in response to suprathreshold tone bursts at CF. Those results demonstrated how single fiber recording allows us to gain important new insights into the development of the cochlea.

To gain insight into the role of synaptic organization in cochlear and ANF function, mice carrying targeted mutations of presynaptic scaffold protein Bassoon were analyzed. Inner 
hair cells (IHC) of mice that are deficient of the central portion of the presynaptic scaffold protein Bassoon $\left(\mathrm{BSN}^{\Delta \mathrm{Ex} 4 / 5}\right)$ were previously shown to mostly lack synaptic ribbons and to have a smaller readily releasable pool of synaptic vesicles and reduced exocytosis, resulting in lower firing rates of ANFs. To distinguish better between the effects of the Bassoon mutation and those of the loss of the synaptic ribbon, the $\mathrm{BSN}^{\Delta \mathrm{Ex} 4 / 5}$ phenotype was compared with that of a newly generated gene trap mutant $\left(\mathrm{BSN}^{\mathrm{gt}}\right)$, which has an intermediate phenotype in terms of the fraction of ribbon occupied active zones, presumably due to leaky expression of a small amount of Bassoon protein. The mean distance between the remaining ribbons and the active zone was greater in $\mathrm{BSN}^{\mathrm{gt}}$ than in wildtype and the synaptic calcium channel clusters had reduced immunostaining reactivity. The $\mathrm{BSN}^{\mathrm{gt}} \mathrm{IHCs}$ showed a slightly less severe reduction of peak $\mathrm{Ca}^{2+}$ currents and sustained exocytosis compared to $\mathrm{BSN}^{\Delta \mathrm{Ex} 4 / 5}$. However, IHC fast exocytosis and single unit responses of ANFs showed almost identical response properties between the two mutants. These data suggest that it is not the physical presence or absence of a synaptic ribbon but rather the disruption of presynaptic ultrastructure (e.g. abnormal calcium channel clustering, looser ribbon anchorage) that mainly determines the synaptic phenotype of Bassoon mutants.

Certain mouse strain suffer from sensorineuronal hearing loss. The ANF responses of Black Swiss mice (BLSW) were characterized. BLSW mice have inherited early onset hearing loss and susceptibility to audiogenic seizures. BLSW ANFs showed higher tuning thresholds and broader frequency selectivity, indicating the presence of $\mathrm{OHC}$ lesions. This observation is consistent with previous data. The unaffected suprathreshold responses to tone bursts suggested that neurotransmission release dynamics is minimally affected in BLSW IHCs. Interestingly, BLSW ANFs have elevated spontaneous discharge activity. Previous results showed that a mutation in the Gipc3 gene is underlying the BLSW phenotypes. The ANF sound encoding deficits in BLSW mice suggest that Gipc3 is a key molecular player for normal IHC and OHC function.

Upon hearing loss due to $\mathrm{HC}$ dysfunction, the remaining ANFs can be electrically stimulated to restore the sense of hearing. The number of useful frequency channels using electrical stimulation is limited by the spread of current. Recently, optical stimulation showed more selective activation of ANFs compared to electrical stimulation. Genetic manipulation in mice makes it possible to express transgenes in certain cell types. ChR2 
and the ChR2-variant CatCh were expressed in spiral ganglion neurons (SGNs) and the optical evoked responses were characterized. It was found that neurons in the auditory pathway can be activated by optogenetic stimulation of mouse cochlea. Neurons expressing CatCh responded to light with more precise spikes and shorter latencies compared to $\mathrm{ChR} 2$ expressing neurons, which is consistent with the published channel properties. 


\section{References}

Abe, T., S. Kakehata, R. Kitani, S. Maruya, D. Navaratnam, J. Santos-Sacchi and H. Shinkawa (2007). "Developmental expression of the outer hair cell motor prestin in the mouse." J Membr Biol 215(1): 49-56.

Alford, B. R. and R. J. Ruben (1963). "Physiological, behavioral and anatomical correlates of the development of hearing in the mouse." Ann Otol Rhinol Laryngol 72: 237-247.

Altrock, W. D., S. tom Dieck, M. Sokolov, A. C. Meyer, A. Sigler, C. Brakebusch, R. Fassler, K. Richter, T. M. Boeckers, H. Potschka, C. Brandt, W. Loscher, D. Grimberg, T. Dresbach, A. Hempelmann, H. Hassan, D. Balschun, J. U. Frey, J. H. Brandstatter, C. C. Garner, C.

Rosenmund and E. D. Gundelfinger (2003). "Functional inactivation of a fraction of excitatory synapses in mice deficient for the active zone protein bassoon." Neuron 37(5): 787-800.

Anniko, M. and R. Wroblewski (1986). "Ionic environment of cochlear hair cells." Hear Res 22: 279-293.

Arenkiel, B. R., J. Peca, I. G. Davison, C. Feliciano, K. Deisseroth, G. J. Augustine, M. D. Ehlers and G. Feng (2007). "In vivo light-induced activation of neural circuitry in transgenic mice expressing channelrhodopsin-2." Neuron 54(2): 205-218.

Ashmore, J. (2008). "Cochlear outer hair cell motility." Physiol Rev 88(1): 173-210.

Berry, M. J., 2nd and M. Meister (1998). "Refractoriness and neural precision." $\underline{\text { J Neurosci }}$ 18(6): 2200-2211.

Beurg, M., N. Michalski, S. Safieddine, Y. Bouleau, R. Schneggenburger, E. R. Chapman, C. Petit and D. Dulon (2010). "Control of exocytosis by synaptotagmins and otoferlin in auditory hair cells." J Neurosci 30(40): 13281-13290.

Beutner, D. and T. Moser (2001). "The presynaptic function of mouse cochlear inner hair cells during development of hearing." J Neurosci 21(13): 4593-4599.

Beutner, D. and T. Moser (2001). "The Presynaptic Function of Mouse Cochlear Inner Hair Cells during Development of Hearing." The Journal of Neuroscience 21(13): 4593-4599.

Blackburn, C. C. and M. B. Sachs (1989). "Classification of unit types in the anteroventral cochlear nucleus: PST histograms and regularity analysis." J Neurophysiol 62(6): 1303-1329.

Blankenship, A. G. and M. B. Feller (2010). "Mechanisms underlying spontaneous patterned activity in developing neural circuits." Nat Rev Neurosci 11(1): 18-29.

Borg, E., B. Engström, G. Linde and K. Marklund (1988). "Eighth nerve fiber firing features in normal-hearing rabbits." Hearing Research 36(2-3): 191-201.

Brandstatter, J. H., E. L. Fletcher, C. C. Garner, E. D. Gundelfinger and H. Wassle (1999). "Differential expression of the presynaptic cytomatrix protein bassoon among ribbon synapses in the mammalian retina." Eur J Neurosci 11(10): 3683-3693.

Brandt, A., D. Khimich and T. Moser (2005). "Few CaV1.3 channels regulate the exocytosis of a synaptic vesicle at the hair cell ribbon synapse." J Neurosci 25(50): 11577-11585. 
Brandt, A., J. Striessnig and T. Moser (2003). "CaV1.3 channels are essential for development and presynaptic activity of cochlear inner hair cells." J Neurosci 23(34): 10832-10840.

Bregman, A. S. (1990). Auditory scene analysis : the perceptual organization of sound. Cambridge, Mass., MIT Press.

Brigande, J. V., S. P. Gubbels, D. W. Woessner, J. J. Jungwirth and C. S. Bresee (2009). "Electroporation-mediated gene transfer to the developing mouse inner ear." Methods Mol Biol 493: $125-139$.

Bulankina, A. V. and T. Moser (2012). "Neural circuit development in the mammalian cochlea." Physiology (Bethesda) 27(2): 100-112.

Buran, B. N., N. Strenzke, A. Neef, E. D. Gundelfinger, T. Moser and M. C. Liberman (2010). "Onset coding is degraded in auditory nerve fibers from mutant mice lacking synaptic ribbons." J Neurosci 30(22): 7587-7597.

Buunen, T. J. and W. S. Rhode (1978). "Responses of fibers in the cat's auditory nerve to the cubic difference tone." J Acoust Soc Am 64(3): 772-781.

Cai, S., W.-L. Ma and E. Young (2009). "Encoding Intensity in Ventral Cochlear Nucleus Following Acoustic Trauma: Implications for Loudness Recruitment." Journal of the Association for Research in Otolaryngology 10(1): 5-22.

Charizopoulou, N., A. Lelli, M. Schraders, K. Ray, M. S. Hildebrand, A. Ramesh, C. R. Srisailapathy, J. Oostrik, R. J. Admiraal, H. R. Neely, J. R. Latoche, R. J. Smith, J. K. Northup, H. Kremer, J. R. Holt and K. Noben-Trauth (2011). "Gipc3 mutations associated with audiogenic seizures and sensorineural hearing loss in mouse and human." Nat Commun 2: 201.

Ciuman, R. R. (2011). "Auditory and vestibular hair cell stereocilia: relationship between functionality and inner ear disease." J Laryngol Otol 125(10): 991-1003.

Clapcote, S. J., N. L. Lazar, A. R. Bechard, G. A. Wood and J. C. Roder (2005). "NIH Swiss and Black Swiss mice have retinal degeneration and performance deficits in cognitive tests." Comp Med 55(4): 310-316.

Dallos, P., X. Wu, M. A. Cheatham, J. Gao, J. Zheng, C. T. Anderson, S. Jia, X. Wang, W. H. Cheng, S. Sengupta, D. Z. He and J. Zuo (2008). "Prestin-based outer hair cell motility is necessary for mammalian cochlear amplification." Neuron 58(3): 333-339.

Dick, O., I. Hack, W. D. Altrock, C. C. Garner, E. D. Gundelfinger and J. H. Brandstatter (2001). "Localization of the presynaptic cytomatrix protein Piccolo at ribbon and conventional synapses in the rat retina: comparison with Bassoon." J Comp Neurol 439(2): 224-234.

Dick, O., S. tom Dieck, W. D. Altrock, J. Ammermuller, R. Weiler, C. C. Garner, E. D. Gundelfinger and J. H. Brandstatter (2003). "The presynaptic active zone protein bassoon is essential for photoreceptor ribbon synapse formation in the retina." Neuron 37(5): 775-786.

Dieck, S. and J. Brandstätter (2006). "Ribbon synapses of the retina." Cell and Tissue Research 326(2): 339-346.

Drayton, M. and K. Noben-Trauth (2006). "Mapping quantitative trait loci for hearing loss in Black Swiss mice." Hear Res 212(1-2): 128-139. 
Eggermont, J. J., G. M. Smith and D. Bowman (1993). "Spontaneous burst firing in cat primary auditory cortex: age and depth dependence and its effect on neural interaction measures." $\mathbf{J}$ Neurophysiol 69(4): 1292-1313.

el Barbary, A. (1991). "Auditory nerve of the normal and jaundiced rat. I. Spontaneous discharge rate and cochlear nerve histology." Hear Res 54(1): 75-90.

el Barbary, A. (1991). "Auditory nerve of the normal and jaundiced rat. II. Frequency selectivity and two-tone rate suppression." Hear Res 54(1): 91-104.

Evans, E. F. (1972). "The frequency response and other properties of single fibres in the guineapig cochlear nerve." J Physiol 226(1): 263-287.

Fenster, S. D., W. J. Chung, R. Zhai, C. Cases-Langhoff, B. Voss, A. M. Garner, U. Kaempf, S. Kindler, E. D. Gundelfinger and C. C. Garner (2000). "Piccolo, a presynaptic zinc finger protein structurally related to bassoon." Neuron 25(1): 203-214.

Frank, T., D. Khimich, A. Neef and T. Moser (2009). "Mechanisms contributing to synaptic $\mathrm{Ca} 2+$ signals and their heterogeneity in hair cells." Proc Natl Acad Sci U S A 106(11): 44834488 .

Frank, T., M. A. Rutherford, N. Strenzke, A. Neef, T. Pangrsic, D. Khimich, A. Fejtova, E. D. Gundelfinger, M. C. Liberman, B. Harke, K. E. Bryan, A. Lee, A. Egner, D. Riedel and T. Moser (2010). "Bassoon and the synaptic ribbon organize $\mathrm{Ca}(2)+$ channels and vesicles to add release sites and promote refilling." Neuron 68(4): 724-738.

Friedman, H. V., T. Bresler, C. C. Garner and N. E. Ziv (2000). "Assembly of new individual excitatory synapses: time course and temporal order of synaptic molecule recruitment." Neuron 27(1): 57-69.

Fuchs, P. A., E. Glowatzki and T. Moser (2003). "The afferent synapse of cochlear hair cells." Curr Opin Neurobiol 13(4): 452-458.

Furukawa, T. and S. Matsuura (1978). "Adaptive rundown of excitatory post-synaptic potentials at synapses between hair cells and eight nerve fibres in the goldfish." J Physiol 276: 193-209.

Geisler, C. D. (1998). From sound to synapse : physiology of the mammalian ear. New York, Oxford University Press.

Giese, A. P., J. Ezan, L. Wang, L. Lasvaux, F. Lembo, C. Mazzocco, E. Richard, J. Reboul, J.P. Borg, M. W. Kelley, N. Sans, J. Brigande and M. Montcouquiol (2012). "Gipc1 has a dual role in Vangl2 trafficking and hair bundle integrity in the inner ear." Development 139(20): 3775-3785.

Glowatzki, E. and P. A. Fuchs (2002). "Transmitter release at the hair cell ribbon synapse." Nat Neurosci 5(2): 147-154.

Goldberg, J. M. and P. B. Brown (1969). "Response of binaural neurons of dog superior olivary complex to dichotic tonal stimuli: some physiological mechanisms of sound localization." J Neurophysiol 32(4): 613-636.

Goutman, J. D. and E. Glowatzki (2007). "Time course and calcium dependence of transmitter release at a single ribbon synapse." Proc Natl Acad Sci U S A 104(41): 16341-16346. 
Grant, L., E. Yi and E. Glowatzki (2010). "Two modes of release shape the postsynaptic response at the inner hair cell ribbon synapse." J Neurosci 30(12): 4210-4220.

Grant, L., E. Yi, J. D. Goutman and E. Glowatzki (2011). "Postsynaptic recordings at afferent dendrites contacting cochlear inner hair cells: monitoring multivesicular release at a ribbon synapse." J Vis Exp(48).

Guinan, J. J., Jr. and M. L. Gifford (1988). "Effects of electrical stimulation of efferent olivocochlear neurons on cat auditory-nerve fibers. II. Spontaneous rate." Hear Res 33(2): 115127.

Gummer, A. W. and R. F. Mark (1994). "Patterned neural activity in brain stem auditory areas of a prehearing mammal, the tammar wallaby (Macropus eugenii)." Neuroreport 5(6): 685-688.

Hallermann, S., A. Fejtova, H. Schmidt, A. Weyhersmuller, R. A. Silver, E. D. Gundelfinger and J. Eilers (2010). "Bassoon speeds vesicle reloading at a central excitatory synapse." Neuron 68(4): $710-723$.

Harrison, R. V. (1981). "Rate-versus-intensity functions and related AP responses in normal and pathological guinea pig and human cochleas." J Acoust Soc Am 70(4): 1036-1044.

Heidrych, P., U. Zimmermann, S. Kuhn, C. Franz, J. Engel, S. V. Duncker, B. Hirt, C. M. Pusch, P. Ruth, M. Pfister, W. Marcotti, N. Blin and M. Knipper (2009). "Otoferlin interacts with myosin VI: implications for maintenance of the basolateral synaptic structure of the inner hair cell." Hum Mol Genet 18(15): 2779-2790.

Heinz, M. G., H. S. Colburn and L. H. Carney (2002). "Quantifying the implications of nonlinear cochlear tuning for auditory-filter estimates." J Acoust Soc Am 111(2): 996-1011.

Heinz, M. G. and E. D. Young (2004). "Response growth with sound level in auditory-nerve fibers after noise-induced hearing loss." J Neurophysiol 91(2): 784-795.

Huttenbrink, K. B. (1989). "[Movement of the ear ossicles by middle ear muscle contraction]." Laryngorhinootologie 68(11): 614-621.

Jing, Z., M. A. Rutherford, H. Takago, T. Frank, A. Fejtova, D. Khimich, T. Moser and N. Strenzke (2013). "Disruption of the Presynaptic Cytomatrix Protein Bassoon Degrades Ribbon Anchorage, Multiquantal Release, and Sound Encoding at the Hair Cell Afferent Synapse." J Neurosci 33(10): 4456-4467.

Johnson, S. L., H. J. Kennedy, M. C. Holley, R. Fettiplace and W. Marcotti (2012). "The Resting Transducer Current Drives Spontaneous Activity in Prehearing Mammalian Cochlear Inner Hair Cells." The Journal of Neuroscience 32(31): 10479-10483.

Johnson, S. L., W. Marcotti and C. J. Kros (2005). "Increase in efficiency and reduction in Ca2+ dependence of exocytosis during development of mouse inner hair cells." J Physiol 563(Pt 1): 177-191.

Jones, T. A., P. A. Leake, R. L. Snyder, O. Stakhovskaya and B. Bonham (2007). "Spontaneous discharge patterns in cochlear spiral ganglion cells before the onset of hearing in cats." J Neurophysiol 98(4): 1898-1908.

Joris, P. X. (2009). "Recruitment of neurons and loudness. Commentary on "Encoding intensity in ventral cochlear nucleus following acoustic trauma: implications for loudness recruitment" by 
Cai et al. J. Assoc. Res. Otolaryngol. DOI: 10.1007/s10162-008-0142-y." J Assoc Res Otolaryngol 10(1): 1-4.

Katoh, M. (2002). "GIPC gene family (Review)." Int J Mol Med 9(6): 585-589.

Khimich, D., R. Nouvian, R. Pujol, S. Tom Dieck, A. Egner, E. D. Gundelfinger and T. Moser (2005). "Hair cell synaptic ribbons are essential for synchronous auditory signalling." Nature 434(7035): 889-894.

Kiang, N. Y.-s. (1965). Discharge patterns of single fibers in the cat's auditory nerve. Cambridge, Mass.,, M.I.T. Press.

Kiang, N. Y., M. C. Liberman, W. F. Sewell and J. J. Guinan (1986). "Single unit clues to cochlear mechanisms." Hear Res 22: 171-182.

Kleinlogel, S., K. Feldbauer, R. E. Dempski, H. Fotis, P. G. Wood, C. Bamann and E. Bamberg (2011). "Ultra light-sensitive and fast neuronal activation with the $\mathrm{Ca}(2)+$-permeable channelrhodopsin CatCh." Nat Neurosci 14(4): 513-518.

Lagali, P. S., D. Balya, G. B. Awatramani, T. A. Munch, D. S. Kim, V. Busskamp, C. L. Cepko and B. Roska (2008). "Light-activated channels targeted to ON bipolar cells restore visual function in retinal degeneration." Nat Neurosci 11(6): 667-675.

Lanore, F., C. Blanchet, A. Fejtova, P. Pinheiro, K. Richter, D. Balschun, E. Gundelfinger and C. Mulle (2010). "Impaired development of hippocampal mossy fibre synapses in mouse mutants for the presynaptic scaffold protein Bassoon." J Physiol 588(Pt 12): 2133-2145.

Liberman, L. D., H. Wang and M. C. Liberman (2011). "Opposing gradients of ribbon size and AMPA receptor expression underlie sensitivity differences among cochlear-nerve/hair-cell synapses." J Neurosci 31(3): 801-808.

Liberman, M. C. (1978). "Auditory-nerve response from cats raised in a low-noise chamber." $\underline{\mathrm{J}}$ Acoust Soc Am 63(2): 442-455.

Liberman, M. C. (1980). "Morphological differences among radial afferent fibers in the cat cochlea: an electron-microscopic study of serial sections." Hear Res 3(1): 45-63.

Liberman, M. C. (1982). "Single-neuron labeling in the cat auditory nerve." Science 216(4551): 1239-1241.

Liberman, M. C. and L. W. Dodds (1984). "Single-neuron labeling and chronic cochlear pathology. III. Stereocilia damage and alterations of threshold tuning curves." Hear Res 16(1): $55-74$.

Liberman, M. C., L. W. Dodds and S. Pierce (1990). "Afferent and efferent innervation of the cat cochlea: quantitative analysis with light and electron microscopy." J Comp Neurol 301(3): 443-460.

Liberman, M. C., J. Gao, D. Z. He, X. Wu, S. Jia and J. Zuo (2002). "Prestin is required for electromotility of the outer hair cell and for the cochlear amplifier." Nature 419(6904): 300-304.

Liberman, M. C. and M. E. Oliver (1984). "Morphometry of intracellularly labeled neurons of the auditory nerve: correlations with functional properties." J Comp Neurol 223(2): 163-176. 
Lippe, W. R. (1994). "Rhythmic spontaneous activity in the developing avian auditory system." J Neurosci 14(3 Pt 2): 1486-1495.

Littlefield, P. D., I. Vujanovic, J. Mundi, A. I. Matic and C. P. Richter (2010). "Laser stimulation of single auditory nerve fibers." Laryngoscope 120(10): 2071-2082.

Møller, A. R. (2006). Hearing : anatomy, physiology, and disorders of the auditory system. Amsterdam ; Boston, Academic Press.

Mardia, K. V. and P. E. Jupp (2000). Directional statistics. Chichester ; New York, J. Wiley.

Melcher, J. R., J. J. Guinan, Jr., I. M. Knudson and N. Y. Kiang (1996). "Generators of the brainstem auditory evoked potential in cat. II. Correlating lesion sites with waveform changes." Hear Res 93(1-2): 28-51.

Melcher, J. R. and N. Y. Kiang (1996). "Generators of the brainstem auditory evoked potential in cat. III: Identified cell populations." Hear Res 93(1-2): 52-71.

Melcher, J. R., I. M. Knudson, B. C. Fullerton, J. J. Guinan, B. E. Norris and N. Y. S. Kiang (1996). "Generators of the brainstem auditory evoked potential in cat .1. An experimental approach to their identification." Hearing Research 93(1-2): 1-27.

Merchan-Perez, A. and M. C. Liberman (1996). "Ultrastructural differences among afferent synapses on cochlear hair cells: correlations with spontaneous discharge rate." J Comp Neurol 371(2): 208-221.

Mikaelian, D., B. R. Alford and R. J. Ruben (1965). "Cochlear Potentials and 8 Nerve Action Potentials in Normal and Genetically Deaf Mice." Ann Otol Rhinol Laryngol 74: 146-157.

Mikaelian, D. and R. J. Ruben (1965). "Development of Hearing in the Normal Cba-J Mouse: Correlation of Physiological Observations with Behavioral Responses and with Cochlear Anatomy." Acta Oto-laryngologica 59(2-6): 451-461.

Misawa, H., E. H. Sherr, D. J. Lee, D. M. Chetkovich, A. Tan, C. E. Schreiner and D. S. Bredt (2002). "Identification of a monogenic locus (jams1) causing juvenile audiogenic seizures in mice." J Neurosci 22(23): 10088-10093.

Moser, T. and D. Beutner (2000). "Kinetics of exocytosis and endocytosis at the cochlear inner hair cell afferent synapse of the mouse." Proc Natl Acad Sci U S A 97(2): 883-888.

Moser, T., A. Neef and D. Khimich (2006). "Mechanisms underlying the temporal precision of sound coding at the inner hair cell ribbon synapse." J Physiol 576(Pt 1): 55-62.

Mukherjee, K., X. Yang, S. H. Gerber, H. B. Kwon, A. Ho, P. E. Castillo, X. Liu and T. C. Sudhof (2010). "Piccolo and bassoon maintain synaptic vesicle clustering without directly participating in vesicle exocytosis." Proc Natl Acad Sci U S A 107(14): 6504-6509.

Muresan, V., A. Lyass and B. J. Schnapp (1999). "The Kinesin Motor KIF3A Is a Component of the Presynaptic Ribbon in Vertebrate Photoreceptors." The Journal of Neuroscience 19(3): $1027-1037$.

Nouvian, R., D. Beutner, T. D. Parsons and T. Moser (2006). "Structure and function of the hair cell ribbon synapse." J Membr Biol 209(2-3): 153-165. 
Oliver, D., A. M. Taberner, H. Thurm, M. Sausbier, C. Arntz, P. Ruth, B. Fakler and M. C. Liberman (2006). "The role of BKCa channels in electrical signal encoding in the mammalian auditory periphery." J Neurosci 26(23): 6181-6189.

Olson, E. S., H. Duifhuis and C. R. Steele (2012). "Von Bekesy and cochlear mechanics." Hear Res 293(1-2): 31-43.

Parsons, T. D. (2006). "Neurobiology: auditory fidelity." Nature 444(7122): 1013-1014.

Parsons, T. D., D. Lenzi, W. Almers and W. M. Roberts (1994). "Calcium-triggered exocytosis and endocytosis in an isolated presynaptic cell: capacitance measurements in saccular hair cells." Neuron 13(4): 875-883.

Patuzzi, R. and P. M. Sellick (1983). "A comparison between basilar membrane and inner hair cell receptor potential input-output functions in the guinea pig cochlea." J Acoust Soc Am 74(6): 1734-1741.

Piatigorsky, J. (2001). "Dual use of the transcriptional repressor (CtBP2)/ribbon synapse (RIBEYE) gene: how prevalent are multifunctional genes?" Trends Neurosci 24(10): 555-557.

Plack, C. J. (2005). The sense of hearing. Mahwah, N.J., Lawrence Erlbaum Associates.

Platzer, J., J. Engel, A. Schrott-Fischer, K. Stephan, S. Bova, H. Chen, H. Zheng and J. Striessnig (2000). "Congenital deafness and sinoatrial node dysfunction in mice lacking class D L-type Ca2+ channels." Cell 102(1): 89-97.

Probst, F. J., R. A. Fridell, Y. Raphael, T. L. Saunders, A. Wang, Y. Liang, R. J. Morell, J. W. Touchman, R. H. Lyons, K. Noben-Trauth, T. B. Friedman and S. A. Camper (1998). "Correction of deafness in shaker-2 mice by an unconventional myosin in a BAC transgene." Science 280(5368): 1444-1447.

Quiroga, R. Q., Z. Nadasdy and Y. Ben-Shaul (2004). "Unsupervised spike detection and sorting with wavelets and superparamagnetic clustering." Neural Comput 16(8): 1661-1687.

Regus-Leidig, H., S. tom Dieck and J. H. Brandstatter (2010). "Absence of functional active zone protein Bassoon affects assembly and transport of ribbon precursors during early steps of photoreceptor synaptogenesis." Eur J Cell Biol 89(6): 468-475.

Regus-Leidig, H., S. Tom Dieck, D. Specht, L. Meyer and J. H. Brandstatter (2009). "Early steps in the assembly of photoreceptor ribbon synapses in the mouse retina: the involvement of precursor spheres." J Comp Neurol 512(6): 814-824.

Rehman, A. U., K. Gul, R. J. Morell, K. Lee, Z. M. Ahmed, S. Riazuddin, R. A. Ali, M. Shahzad, A. U. Jaleel, P. B. Andrade, S. N. Khan, S. Khan, C. C. Brewer, W. Ahmad, S. M. Leal, S. Riazuddin and T. B. Friedman (2011). "Mutations of GIPC3 cause nonsyndromic hearing loss DFNB72 but not DFNB81 that also maps to chromosome 19p." Hum Genet 130(6): 759-765.

Reisinger, E., C. Bresee, J. Neef, R. Nair, K. Reuter, A. Bulankina, R. Nouvian, M. Koch, J. Buckers, L. Kastrup, I. Roux, C. Petit, S. W. Hell, N. Brose, J. S. Rhee, S. Kugler, J. V. Brigande and T. Moser (2011). "Probing the functional equivalence of otoferlin and synaptotagmin 1 in exocytosis." J Neurosci 31(13): 4886-4895. 
Richter, K., K. Langnaese, M. R. Kreutz, G. Olias, R. Zhai, H. Scheich, C. C. Garner and E. D. Gundelfinger (1999). "Presynaptic cytomatrix protein bassoon is localized at both excitatory and inhibitory synapses of rat brain." J Comp Neurol 408(3): 437-448.

Robertson, D. and B. Paki (2002). "Role of L-type Ca2+ channels in transmitter release from mammalian inner hair cells. II. Single-neuron activity." J Neurophysiol 87(6): 2734-2740.

Robles, L. and M. A. Ruggero (2001). "Mechanics of the mammalian cochlea." Physiol Rev 81(3): 1305-1352.

Romand, R. (1983). "Development in the frequency selectivity of auditory nerve fibers in the kitten." Neurosci Lett 35(3): 271-276.

Romand, R. (1984). "Functional properties of auditory-nerve fibers during postnatal development in the kitten." Exp Brain Res 56(3): 395-402.

Roux, I., S. Hosie, S. L. Johnson, A. Bahloul, N. Cayet, S. Nouaille, C. J. Kros, C. Petit and S. Safieddine (2009). "Myosin VI is required for the proper maturation and function of inner hair cell ribbon synapses." Hum Mol Genet 18(23): 4615-4628.

Russell, I. J. and P. M. Sellick (1978). "Intracellular studies of hair cells in the mammalian cochlea." J Physiol 284: 261-290.

Rutherford, M. A., N. M. Chapochnikov and T. Moser (2012). "Spike encoding of neurotransmitter release timing by spiral ganglion neurons of the cochlea." J Neurosci 32(14): 4773-4789.

Sachs, M. B. and P. J. Abbas (1974). "Rate versus level functions for auditory-nerve fibers in cats: tone-burst stimuli." J Acoust Soc Am 56(6): 1835-1847.

Sachs, M. B., R. L. Winslow and B. H. Sokolowski (1989). "A computational model for ratelevel functions from cat auditory-nerve fibers." Hear Res 41(1): 61-69.

Sadanaga, M. and T. Morimitsu (1995). "Development of endocochlear potential and its negative component in mouse cochlea." Hear Res 89(1-2): 155-161.

Saunders, J. C., K. G. Dolgin and L. D. Lowry (1980). "The maturation of frequency selectivity in C57BL/6J mice studied with auditory evoked response tuning curves." Brain Res 187(1): 6979.

Schmitz, F. (2009). "The making of synaptic ribbons: how they are built and what they do." Neuroscientist 15(6): 611-624.

Schnupp, J., I. Nelken and A. King (2011). Auditory neuroscience : making sense of sound. Cambridge, Mass., MIT Press.

Self, T., M. Mahony, J. Fleming, J. Walsh, S. D. Brown and K. P. Steel (1998). "Shaker-1 mutations reveal roles for myosin VIIA in both development and function of cochlear hair cells." Development 125(4): 557-566.

Sewell, W. F. (1984). "The relation between the endocochlear potential and spontaneous activity in auditory nerve fibres of the cat." J Physiol 347: 685-696. 
Shapira, M., R. G. Zhai, T. Dresbach, T. Bresler, V. I. Torres, E. D. Gundelfinger, N. E. Ziv and C. C. Garner (2003). "Unitary assembly of presynaptic active zones from Piccolo-Bassoon transport vesicles." Neuron 38(2): 237-252.

Sheets, L., J. G. Trapani, W. Mo, N. Obholzer and T. Nicolson (2011). "Ribeye is required for presynaptic $\mathrm{Ca}(\mathrm{V}) 1.3$ a channel localization and afferent innervation of sensory hair cells." Development 138(7): 1309-1319.

Siegel, J. H. (1992). "Spontaneous synaptic potentials from afferent terminals in the guinea pig cochlea." Hear Res 59(1): 85-92.

Siegel, J. H. and E. M. Relkin (1987). "Antagonistic effects of perilymphatic calcium and magnesium on the activity of single cochlear afferent neurons." Hear Res 28(2-3): 131-147.

Singer, J. H. (2007). "Multivesicular release and saturation of glutamatergic signalling at retinal ribbon synapses." J Physiol 580(Pt 1): 23-29.

Singer, J. H. and J. S. Diamond (2006). "Vesicle depletion and synaptic depression at a mammalian ribbon synapse." J Neurophysiol 95(5): 3191-3198.

Singer, J. H., L. Lassova, N. Vardi and J. S. Diamond (2004). "Coordinated multivesicular release at a mammalian ribbon synapse." Nat Neurosci 7(8): 826-833.

Snellman, J., B. Mehta, N. Babai, T. M. Bartoletti, W. Akmentin, A. Francis, G. Matthews, W. Thoreson and D. Zenisek (2011). "Acute destruction of the synaptic ribbon reveals a role for the ribbon in vesicle priming." Nat Neurosci 14(9): 1135-1141.

Sobkowicz, H. M., J. E. Rose, G. E. Scott and S. M. Slapnick (1982). "Ribbon synapses in the developing intact and cultured organ of Corti in the mouse." J Neurosci 2(7): 942-957.

Song, L., J. McGee and E. J. Walsh (2006). "Frequency- and level-dependent changes in auditory brainstem responses (ABRS) in developing mice." J Acoust Soc Am 119(4): 2242 2257.

Song, L., J. McGee and E. J. Walsh (2008). "Development of cochlear amplification, frequency tuning, and two-tone suppression in the mouse." J Neurophysiol 99(1): 344-355.

Spassova, M. A., M. Avissar, A. C. Furman, M. A. Crumling, J. C. Saunders and T. D. Parsons (2004). "Evidence that rapid vesicle replenishment of the synaptic ribbon mediates recovery from short-term adaptation at the hair cell afferent synapse." J Assoc Res Otolaryngol 5(4): 376390.

Steel, K. P. (1991). "Similarities between mice and humans with hereditary deafness." Ann N Y Acad Sci 630: 68-79.

Steel, K. P. (1995). "Inherited hearing defects in mice." Annu Rev Genet 29: 675-701.

Stillman, J. A., J. J. Zwislocki, M. Zhang and L. K. Cefaratti (1993). "Intensity just-noticeable differences at equal-loudness levels in normal and pathological ears." J Acoust Soc Am 93(1): 425-434.

Strenzke, N., S. Chanda, C. Kopp-Scheinpflug, D. Khimich, K. Reim, A. V. Bulankina, A. Neef, F. Wolf, N. Brose, M. A. Xu-Friedman and T. Moser (2009). "Complexin-I is required for high-fidelity transmission at the endbulb of held auditory synapse." J Neurosci 29(25): 79918004 . 
Sumner, C. J., E. A. Lopez-Poveda, L. P. O'Mard and R. Meddis (2002). "A revised model of the inner-hair cell and auditory-nerve complex." J Acoust Soc Am 111(5 Pt 1): 2178-2188.

Sumner, C. J., E. A. Lopez-Poveda, L. P. O'Mard and R. Meddis (2003). "Adaptation in a revised inner-hair cell model." J Acoust Soc Am 113(2): 893-901.

Taberner, A. M. and M. C. Liberman (2005). "Response properties of single auditory nerve fibers in the mouse." J Neurophysiol 93(1): 557-569.

tom Dieck, S., W. D. Altrock, M. M. Kessels, B. Qualmann, H. Regus, D. Brauner, A. Fejtova, O. Bracko, E. D. Gundelfinger and J. H. Brandstatter (2005). "Molecular dissection of the photoreceptor ribbon synapse: physical interaction of Bassoon and RIBEYE is essential for the assembly of the ribbon complex." J Cell Biol 168(5): 825-836.

tom Dieck, S., L. Sanmarti-Vila, K. Langnaese, K. Richter, S. Kindler, A. Soyke, H. Wex, K. H. Smalla, U. Kampf, J. T. Franzer, M. Stumm, C. C. Garner and E. D. Gundelfinger (1998). "Bassoon, a novel zinc-finger CAG/glutamine-repeat protein selectively localized at the active zone of presynaptic nerve terminals." J Cell Biol 142(2): 499-509.

Tomita, H., E. Sugano, H. Isago, T. Hiroi, Z. Wang, E. Ohta and M. Tamai (2010).

"Channelrhodopsin-2 gene transduced into retinal ganglion cells restores functional vision in genetically blind rats." Exp Eye Res 90(3): 429-436.

Tritsch, N. X., A. Rodriguez-Contreras, T. T. Crins, H. C. Wang, J. G. Borst and D. E. Bergles (2010). "Calcium action potentials in hair cells pattern auditory neuron activity before hearing onset." Nat Neurosci 13(9): 1050-1052.

Tritsch, N. X., E. Yi, J. E. Gale, E. Glowatzki and D. E. Bergles (2007). "The origin of spontaneous activity in the developing auditory system." Nature 450(7166): 50-55.

Uthaiah, R. C. and A. J. Hudspeth (2010). "Molecular Anatomy of the Hair Cell's Ribbon Synapse." The Journal of Neuroscience 30(37): 12387-12399.

Viemeister, N. F. (1983). "Auditory intensity discrimination at high frequencies in the presence of noise." Science 221(4616): 1206-1208.

von Gersdorff, H., E. Vardi, G. Matthews and P. Sterling (1996). "Evidence that vesicles on the synaptic ribbon of retinal bipolar neurons can be rapidly released." Neuron 16(6): 1221-1227.

Waites, C. L., S. A. Leal-Ortiz, N. Okerlund, H. Dalke, A. Fejtova, W. D. Altrock, E. D. Gundelfinger and C. C. Garner (2013). "Bassoon and Piccolo maintain synapse integrity by regulating protein ubiquitination and degradation." EMBO J.

Wald, A. (1947). Sequential analysis. New York,

London,, J. Wiley \& sons

Chapman \& Hall.

Walsh, E. J. and J. McGee (1987). "Postnatal development of auditory nerve and cochlear nucleus neuronal responses in kittens." Hear Res 28(1): 97-116.

Walsh, E. J., J. McGee and E. Javel (1986). "Development of auditory-evoked potentials in the cat. I. Onset of response and development of sensitivity." J Acoust Soc Am 79(3): 712-724. 
Walsh, E. J., J. McGee and E. Javel (1986). "Development of auditory-evoked potentials in the cat. II. Wave latencies." J Acoust Soc Am 79(3): 725-744.

Walsh, E. J., J. McGee and E. Javel (1986). "Development of auditory-evoked potentials in the cat. III. Wave amplitudes." J Acoust Soc Am 79(3): 745-754.

Wen, B., G. I. Wang, I. Dean and B. Delgutte (2009). "Dynamic range adaptation to sound level statistics in the auditory nerve." J Neurosci 29(44): 13797-13808.

Westerman, L. A. and R. L. Smith (1984). "Rapid and short-term adaptation in auditory nerve responses." Hear Res 15(3): 249-260.

Willott, J. F. (2001). Handbook of mouse auditory research : from behavior to molecular biology. Boca Raton, CRC Press.

Wong, A. B., Z. Jing, M. A. Rutherford, T. Frank, N. Strenzke and T. Moser (2013).

"Concurrent Maturation of Inner Hair Cell Synaptic Ca2+ Influx and Auditory Nerve Spontaneous Activity around Hearing Onset in Mice." J Neurosci 33(26): 10661-10666.

Yates, G. K. (1990). "Basilar membrane nonlinearity and its influence on auditory nerve rateintensity functions." Hear Res 50(1-2): 145-162.

Yates, G. K., D. Robertson and B. M. Johnstone (1985). "Very rapid adaptation in the guinea pig auditory nerve." Hear Res 17(1): 1-12.

Yates, G. K., I. M. Winter and D. Robertson (1990). "Basilar membrane nonlinearity determines auditory nerve rate-intensity functions and cochlear dynamic range." Hear Res 45(3): 203-219.

Zhai, R., G. Olias, W. J. Chung, R. A. Lester, S. tom Dieck, K. Langnaese, M. R. Kreutz, S. Kindler, E. D. Gundelfinger and C. C. Garner (2000). "Temporal appearance of the presynaptic cytomatrix protein bassoon during synaptogenesis." Mol Cell Neurosci 15(5): 417-428. 


\section{Acknowledgement}

During my graduate study in Göttingen, there are so many people who helped and supported me. From whom I learned how to develop into a qualified scientist and a better person. I am grateful to have the precious opportunity to pursuit my study with so many outstanding scientists and future scientists. I would like to express my deep gratitude to them.

First, I would like to acknowledge Dr. Nicola Strenzke and Prof. Tobias Moser, who introduced into this fascinating world of auditory neurophysiology. Thanks to Prof. Tobias Moser for giving me opportunity to work in this laboratory. I would like to thank him for support on my work, and also guidance and rich scientific discussions during the process of my projects. Thanks to Dr. Nicola Strenzke, for supervising me conducting single unit recording studies. Her excellent experimental skills and successfully balanced life as an experimental scientist and also a clinician impressed me very much. I am grateful to her for teaching me to develop into a good experimentalist and an independent scientist.

I would like to thank Prof. Fred Wolf and Prof. Martin Göpfert for being my thesis committee members and their constant support of my work. The knowledge and expertise of them was invaluable to me. Thanks to them for rich discussions, useful feedbacks about my study during committee meetings.

I would like to thank Dr. Mark Rutherford, Dr. Victor Hernandez and Dr. Thomas Frank for fruitful scientific cooperation. I learned from them different experimental methodologies. From them, I learned to develop a more systematic understanding of the system that I am working on. Thanks to Dr. Sangyong Jung for cell physiology experiments on BLSW project.

Thanks to Gerhard Hoch, the electrical engineer of the lab, for providing the excellent technique support, helping to establish and maintain all the setups and programing MATLAB, all of which are essential for my work. Thanks to Nadine Herrmann and Stefan Thom for technical support on ABR measurement. Christian Senger-Freitag and Sandra Gerke for technical support on mice genotyping.

I would also like to thank members of InnerEarLab, where I met so many ingenious young scientists. Thanks for lively discussions and inspiring ideas during lab seminars and journal clubs. It is a wonderful experience working in such an environment. The diverse background and expertise of each member inspired me to understand scientific problem from different viewpoint. The scientific and personal connections with colleagues are invaluable for me. They are: Aaron Wong, Alejandro M. Schulz, Alexandra Müller, Anna Gehrt, Chao-Hua Huang, Carolin 
Wichmann, Christiane Vogl, Ellen Reisinger, Elisabeth Auge, Gulnara Yamanbaeva, Iliana Panou, Jakob Neef, Hideki Takago, Maria Picher, Marcus Jeschke, Nicolai M. Chapochnikov, Rituparna Chakrabarti, Sarah Helfmann, Susann Michanski, Tanvi Butola, Tina Pangric, Tomoko Oshima-Takago, Tzu-Lun Wang, Ursula Stalmann. Thanks to Lin-ta Hsu, Ana Jukneviciute, Franciska Hudemann, Devann Rutherford and Brigtte Higazi for coordinate nice lab events and their support to me work.

I furthermore acknowledge Prof. Michael Hörner and Sandra Drube from IMPRS Neurosciences office, for their constant support to young students, in both living and academic work. Thanks for their excellent work organizing the Neurosciences program and coordinate social and scientific events to promoting interactions between young scientists from different field.

I am deeply grateful for the constant support from my parents, extended family members and friends for their understanding and encouragement. Their love, support and care will always be a motivator for me to become a better person. 


\section{Curriculum Vitae}

Name:

Citizenship:

Date of Birth:

Place of Birth:

\section{$\underline{\text { Education }}$}

$2004-2008$

$2008-2009$

$2009-2010$

$2010-2013$
Zhizi Jing

People's Republic of China

$6^{\text {th }}$ Nov, 1985

Chongqing, People's Republic of China

B.S. in Biological Science, College of Life Sciences, NanKai University, Tianjin, P.R.China

M.Sc. study year in International Max-Planck Research School in Neurosciences, University of Göttingen, Germany

M.Sc. in Neurosciences, M.Sc thesis with Dr. Nicola Strenzke and Prof. Tobias Moser, InnerEarLab, Department of Otolaryngology, University Medicine Göttingen

$\mathrm{PhD}$ student, $\mathrm{PhD}$ thesis with Dr. Nicola Strenzke, auditory system physiology group, InnerEarLab, Department of Otolaryngolgoy, University Medical Center Göttingen 


\section{Publication List}

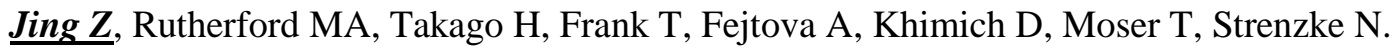
(2013). "Disruption of the presynaptic cytomatrix protein bassoon degrades ribbon anchorage, multiquantal release, and sound encoding at the hair cell afferent synapse." J Neurosci 33(10): 4456-4467.

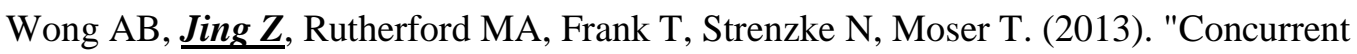
Maturation of Inner Hair Cell Synaptic $\mathrm{Ca}^{2+}$ Influx and Auditory Nerve Spontaneous Activity around Hearing Onset in Mice." J Neurosci 33(26): 10661-10666.

Mendoza Schulz A, $\underline{\text { Jing Z Z }}$, María Sánchez Caro J, Strenzke N, Wichmann C, Moser T. (2013) "Bassoon-disruption slows vesicle replenishment and induces homeostatic plasticity at a central auditory synapse". EMBO J doi:10.1002/embj.201385887.

Hernadez VH, Gehrt A, Reuter K, Jing Z , Mendoza Schulz A, Hoch G, Bartels M, Yawo H, Fukazawa Y, Augustine GJ, Bamberg E, Kügler S, Salditt T, Strenzke N, Moser T. (2013) "Optogenetic stimulation of the auditory nerve". J Clin Invest doi:10.1172/JCI69050. 